\title{
The Keck Baryonic Structure Survey: using foreground/background galaxy pairs to trace the structure and kinematics of circumgalactic neutral hydrogen at $z \sim 2$
}

\author{
Yuguang Chen(陈昱光) ${ }^{\circledR 1},{ }^{\star}$ Charles C. Steidel $^{\circledR},{ }^{1}$ Cameron B. Hummels ${ }^{\circledR},{ }^{1}$ Gwen C. Rudie ${ }^{(0)},{ }^{2}$ \\ Bili Dong(董比立) ${ }^{3}$ Ryan F. Trainor ${ }^{\circledR},{ }^{4}$ Milan Bogosavljević ${ }^{\circledR}, 5$ Dawn K. Erb ${ }^{\circledR}, 6$ Max Pettini ${ }^{\circledR},{ }^{7,8}$ \\ Naveen A. Reddy ${ }^{\circledR}, 9$ Alice E. Shapley ${ }^{\circledR},{ }^{10}$ Allison L. Strom ${ }^{\circledR},{ }^{2}$ Rachel L. Theios ${ }^{\circledR}, 1$ \\ Claude-André Faucher-Giguère ${ }^{\circledR}, 11$ Philip F. Hopkins ${ }^{\circledR 1}$ and Dušan Kereš ${ }^{\circledR 3}$ \\ ${ }^{1}$ Cahill Center for Astronomy and Astrophysics, California Institute of Technology, MC249-17, Pasadena, CA 91125, USA \\ ${ }^{2}$ The Observatories of the Carnegie Institution for Science, 813 Santa Barbara Street, Pasadena, CA 91101, USA \\ ${ }^{3}$ Department of Physics, Center for Astrophysics and Space Sciences, University of California at San Diego, 9500 Gilman Drive, La Jolla, CA 92093, USA \\ ${ }^{4}$ Department of Physics and Astronomy, Franklin \& Marshall College, 637 College Avenue, Lancaster, PA 17603, USA \\ ${ }^{5}$ Division of Science, New York University Abu Dhabi, PO Box 129188, Abu Dhabi, United Arab Emirates \\ ${ }^{6}$ The Leonard E. Parker Center for Gravitation, Cosmology and Astrophysics, Department of Physics, University of Wisconsin-Milwaukee, 3135 North \\ Maryland Avenue, Milwaukee, WI 53211, USA \\ ${ }^{7}$ Institute of Astronomy, University of Cambridge, Madingley Road, Cambridge CB3 OHA, UK \\ ${ }^{8}$ Kavli Institute for Cosmology, University of Cambridge, Madingley Road, Cambridge CB3 OHA, UK \\ ${ }^{9}$ Department of Physics and Astronomy, University of California, Riverside, 900 University Avenue, Riverside, CA 92521, USA \\ ${ }^{10}$ Department of Physics and Astronomy, University of California, Los Angeles, 430 Portola Plaza, Los Angeles, CA 90095, USA \\ ${ }^{11}$ Department of Physics and Astronomy and Center for Interdisciplinary Exploration and Research in Astrophysics (CIERA), Northwestern University, 2145 \\ Sheridan Road, Evanston, IL 60208, USA
}

Accepted 2020 September 10. Received 2020 August 31; in original form 2020 April 30

\begin{abstract}
We present new measurements of the spatial distribution and kinematics of neutral hydrogen in the circumgalactic and intergalactic medium surrounding star-forming galaxies at $z \sim 2$. Using the spectra of $\simeq 3000$ galaxies with redshifts $\langle z\rangle=2.3 \pm 0.4$ from the Keck Baryonic Structure Survey, we assemble a sample of more than 200000 distinct foreground-background pairs with projected angular separations of 3-500 arcsec and spectroscopic redshifts, with $\left\langle z_{\mathrm{fg}}\right\rangle=2.23$ and $\left\langle z_{\mathrm{bg}}\right\rangle=2.57$ (foreground, background redshifts, respectively.) The ensemble of sightlines and foreground galaxies is used to construct a 2D map of the mean excess $\mathrm{H} \mathrm{I}$ Ly $\alpha$ optical depth relative to the intergalactic mean as a function of projected galactocentric distance $\left(20 \lesssim D_{\operatorname{tran}} / \mathrm{pkpc} \lesssim 4000\right)$ and line-of-sight velocity. We obtain accurate galaxy systemic redshifts, providing significant information on the line-of-sight kinematics of $\mathrm{H}$ I gas as a function of projected distance $D_{\text {tran }}$. We compare the map with cosmological zoom-in simulation, finding qualitative agreement between them. A simple two-component (accretion, outflow) analytical model generally reproduces the observed line-of-sight kinematics and projected spatial distribution of H I. The best-fitting model suggests that galaxy-scale outflows with initial velocity $v_{\text {out }} \simeq 600 \mathrm{~km} \mathrm{~s}^{-1}$ dominate the kinematics of circumgalactic H I out to $D_{\text {tran }} \simeq 50 \mathrm{kpc}$, while H I at $D_{\text {tran }} \gtrsim 100 \mathrm{kpc}$ is dominated by infall with characteristic $v_{\text {in }} \lesssim$ circular velocity. Over the impact parameter range $80 \lesssim D_{\text {tran }} / \mathrm{pkpc}$ $\lesssim 200$, the H I line-of-sight velocity range reaches a minimum, with a corresponding flattening in the rest-frame Ly $\alpha$ equivalent width. These observations can be naturally explained as the transition between outflow-dominated and accretion-dominated flows. Beyond $D_{\text {tran }} \simeq 300 \mathrm{pkpc}(\sim 1 \mathrm{cMpc})$, the line-of-sight kinematics are dominated by Hubble expansion.
\end{abstract}

Key words: galaxies: evolution-galaxies: high-redshift-galaxies: ISM-galaxies: intergalatic medium.

\section{INTRODUCTION}

Galaxy formation involves a continuous competition between gas cooling and accretion on the one hand, and feedback-driven heating and/or mass outflows on the other. The outcome of this competition, as a function of time, controls nearly all observable properties of galaxies: e.g. the star formation rate, the fraction of galactic baryons

^E-mail: yuguangchen@astro.caltech.edu converted to stars over the galaxy lifetime, and the fraction of baryons that remain bound to the galaxy. This competition eventually halts star formation and the growth of supermassive black hole mass. The exchange of gaseous baryons between the diffuse intergalactic medium (IGM) and the central regions of galaxies (the interstellar medium; ISM) involves an intermediate baryonic reservoir that has come to be called the 'circumgalactic medium' (CGM; e.g. Steidel et al. 2010; Rudie, Steidel \& Pettini 2012b; Tumlinson, Peeples \& Werk 2017). 
Although there is not yet a consensus, one possible working definition of the CGM is the region containing gas that is outside of the interstellar medium of a galaxy, but that is close enough that the physics and chemistry of the gas and that of the central galaxy are causally connected. For example, the CGM may be (1) the baryonic reservoir that supplies gas, via accretion, to the central regions of the galaxy, providing fuel for star formation and black hole growth; (2) the CGM may also consist of gas that has already been part of the ISM at some point in the past, but has since been dispersed or ejected to large galactocentric radii; or (3) the physical state of the gas can be otherwise affected by energetic processes (mechanical or radiative) originating in the galaxy's central regions, e.g. via galactic winds, radiation pressure, ionization, etc. Therefore, the CGM represents a galaxy's evolving 'sphere of influence'.

Since being postulated by Bahcall \& Spitzer (1969) more than $50 \mathrm{yr}$ ago, evidence for extended $(\sim 100 \mathrm{pkpc})$ haloes of highly ionized, metal-enriched gas around galaxies has continuously accumulated. In recent years, there has been increasing attention given to understanding the physics and chemistry of CGM gas as a function of galaxy properties, e.g. environment (Johnson, Chen \& Mulchaey 2015; Burchett et al. 2016; Nielsen et al. 2018), mass and star formation rate (Adelberger et al. 2005b; Chen et al. 2010; Tumlinson et al. 2011; Rakic et al. 2012; Johnson et al. 2017; Rubin et al. 2018), and cosmic epoch (Hafen et al. 2019; Hummels et al. 2019; Nelson et al. 2019). In large part, the increased focus on the CGM is attributable to a growing appreciation that diffuse gas outside of galaxies is a laboratory where many of the most important, but poorly understood, baryonic processes can be observed and tested.

Redshifts near the peak of cosmic star formation history, at $z$ $\simeq 2-3$ (Madau \& Dickinson 2014), are especially attractive for observations of galaxies and their associated diffuse CGM/IGM gas, due to the accessibility of spectroscopic diagnostics in the rest-frame far-UV (observed optical) and rest-frame optical (observed near-IR) using large ground-based telescopes (see e.g. Steidel et al. 2014). The most sensitive measurements of neutral hydrogen and metals in diffuse gas in the outer parts of galaxies along the line of sight require high resolution (FWHM $\lesssim 10 \mathrm{~km} \mathrm{~s}^{-1}$ ), high signal-to-noise ratio (SNR) of bright background continuum sources - i.e. quasi-stellar objects (QSOs). However, QSOs bright enough to be observed in this way are extremely rare, thereby limiting the number of galaxies whose CGM can be probed. Moreover, each sightline to a suitable background QSO provides at most a single sample, at a single galactocentric distance, for any identified foreground galaxy. This inefficiency makes the assembly of a statistical picture of the CGM/IGM around galaxies at a particular redshift, or having particular properties, very challenging.

Improved efficiency for such QSO sightline surveys can be realized by conducting deep galaxy surveys in regions of the sky selected to include the lines of sight to one or more background QSOs, with emission redshifts chosen to optimize the information content of absorption lines in the QSO spectrum given the galaxy redshift range targeted by the survey (e.g. Lanzetta et al. 1995; Chen et al. 2001; Adelberger et al. 2003, 2005b; Morris \& Jannuzi 2006; Crighton et al. 2011; Prochaska et al. 2011.) The Keck Baryonic Structure Survey (KBSS ${ }^{1}{ }^{1}$ Rudie et al. 2012a; Steidel et al. 2014; Strom et al. 2017) was designed along these lines, specifically to provide a densely sampled spectroscopic survey of star-forming (SF) galaxies in the

${ }^{1}$ The complete spectroscopic catalogues of the galaxies used in this paper and the processed data can be found at the KBSS website: http://ramekin.ca ltech.edu/KBSS. primary redshift range $1.9 \lesssim z_{\text {gal }} \lesssim 2.7$ in 15 survey regions, each of which is centred around the line of sight to a very bright QSO with $z \sim 2.7-2.8$. The Keck/HIRES spectra of the QSOs, together with the positions and redshifts of the galaxies in each survey region, have been analysed in detail to measure neutral hydrogen $(\mathrm{HI})$ and metals associated with the foreground galaxies. Absorption has been measured as a function of projected galactocentric distance to the QSO sightline and as a function of line-of-sight velocity with respect to the galaxy systemic redshift, using both Voigt profile fitting (Rudie et al. 2012a, 2013, 2019) and 'pixel optical depth' techniques (Rakic et al. 2012, 2013; Turner et al. 2014, 2015). These studies have shown that there is H I and C IV significantly in excess of the intergalactic mean extending to at least 2.5 physical Mpc around identified galaxies, but with the most prominent excess of both H I and metals lying within $D_{\text {tran }} \sim 200-300 \mathrm{pkpc}$ and $\Delta v_{\text {LOS }}$ $\lesssim 300-700 \mathrm{~km} \mathrm{~s}^{-1}$. The statistical inferences were based on $\sim 900$ QSO/galaxy pairs with projected separation $D_{\text {tran }}<3 \mathrm{Mpc}$, but only (90, 26, and 10) sample the CGM within $D_{\text {tran }} \leq(500,200$, and 100) pkpc. Thus, in spite of the large observational effort behind KBSS, the statistics of diffuse gas surrounding $z \simeq 2-2.7$ galaxies is limited to relatively small samples within the inner CGM.

Alternatively, as shown by Steidel et al. (2010, hereafter S2010; see also Adelberger et al. 2005b), it is also possible to use the grid of background galaxies - which comes 'for free' with a densely sampled spectroscopic survey - to vastly increase the number of line-of-sight sampling the CGM of foreground galaxies, particularly for small transverse distances (or impact parameter, $D_{\text {tran }} \lesssim 500$ pkpc.) The penalty for increased spatial sampling is, unavoidably, the vastly reduced spectral resolution and SNR - and the associated loss of the ability to resolve individual components and measure column densities along individual sightlines, compared to the HIRES QSO spectra. S2010 used a set of $\sim 500$ galaxy foreground/background angular pairs with separation $\theta \leq 15$ arcsec to trace the rest-frame equivalent width of $\operatorname{Ly} \alpha$ and several strong metal lines as a function of impact parameter over the range $20 \leq D_{\text {tran }} / \mathrm{pkpc} \leq 125$ at $\langle z\rangle=2.2$. In this paper, we extend the methods of S2010, with significant improvements in both the size and quality of the galaxy sample, to characterize $\mathrm{H}$ I absorption over the full range of 20-4000 pkpc. Compared to the earlier KBSS QSO/galaxy pairs, the new galaxy/galaxy analysis includes $\sim 3000$ galaxies, with a factor $>100$ increase in the number of sightlines sampled with $D_{\text {tran }} \leq 500 \mathrm{pkpc}$.

As discussed by S2010, background galaxies are spatially extended, ${ }^{2}$ unlike QSOs, and thus each absorption line probe is in effect averaging over a spatially extended line of sight through the circumgalactic gas associated with the foreground galaxies. CGM gas is known to be clumpy, with indications that the degree of 'clumpiness' (i.e. the size scale on which significant variations of the ionic column density are observed) depends on ionization level, with low-ionization species having smaller coherence scales (see Rauch, Sargent \& Barlow 1999; Rudie et al. 2019). In general, this means that the strength of an absorption feature produced by gas in a foreground galaxy as recorded in the spectrum of a background galaxy will depend on three factors: the fraction of the beam covered by a significant column of the species, the column density in the beam, and the range of line-of-sight velocity $\left(v_{\mathrm{LOS}}\right)$ sampled by the roughly cylindrical volume through the CGM. The dynamic range in total H I column density measurable using stacks of background

\footnotetext{
${ }^{2}$ Typical galaxies in the spectroscopic sample have physical sizes of $d \simeq$ $4 \mathrm{kpc}$. The diameter of the beam as it traverses a galaxy with $z_{\mathrm{fg}} \simeq z_{\text {bg }}-0.3$ would have a similar physical extent.
} 
Table 1. Field-by-field summary of the properties of the KBSS galaxy pair sample.

\begin{tabular}{|c|c|c|c|c|c|c|c|}
\hline $\begin{array}{l}\text { Field } \\
\text { Name }\end{array}$ & $\begin{array}{c}\mathrm{RA}^{a} \\
(\mathrm{~J} 2000.0)\end{array}$ & $\begin{array}{c}\text { Dec }^{a} \\
(\mathrm{~J} 2000.0)\end{array}$ & $\begin{array}{c}\text { Area }^{b} \\
\left(\operatorname{arcmin}^{2}\right)\end{array}$ & $\begin{array}{c}N_{\mathrm{gal}} \\
(z>1.9)\end{array}$ & $\begin{array}{c}N_{\text {pair }}{ }^{c} \\
\left(\text { Full } / z_{\text {neb }}\right)^{d}\end{array}$ & $\begin{array}{c}\left\langle z_{\mathrm{fg}}\right\rangle \\
\left(\mathrm{Full} / z_{\mathrm{neb}}\right)^{d}\end{array}$ & $\begin{array}{c}\Delta z_{\mathrm{fb}} /\left(1+z_{\mathrm{fg}}\right) \\
\left(\mathrm{Full} / z_{\mathrm{neb}}\right)^{d}\end{array}$ \\
\hline Q0100 & 01:03:11 & $+13: 16: 27$ & $7.6 \times 5.6$ & 153 & $762 / 441$ & $2.11 / 2.11$ & $0.108 / 0.111$ \\
\hline Q0105 & 01:08:08 & $+16: 35: 30$ & $7.4 \times 5.3$ & 137 & $519 / 296$ & $2.15 / 2.12$ & $0.103 / 0.108$ \\
\hline Q0142 & $01: 45: 15$ & $-09: 45: 30$ & $7.2 \times 5.2$ & 131 & $572 / 320$ & $2.21 / 2.22$ & $0.113 / 0.116$ \\
\hline Q0207 & 02:09:52 & $-00: 05: 22$ & $7.0 \times 5.4$ & 133 & $480 / 296$ & $2.15 / 2.15$ & $0.130 / 0.140$ \\
\hline Q0449 & $04: 52: 14$ & $-16: 40: 29$ & $6.5 \times 5.0$ & 128 & $561 / 297$ & $2.28 / 2.23$ & $0.108 / 0.105$ \\
\hline Q0821 & $08: 21: 05$ & $+31: 07: 42$ & $7.3 \times 5.5$ & 124 & $413 / 242$ & $2.35 / 2.35$ & $0.112 / 0.113$ \\
\hline Q1009 & 10:11:55 & $+29: 41: 36$ & $7.2 \times 5.2$ & 141 & $552 / 325$ & $2.44 / 2.31$ & $0.125 / 0.129$ \\
\hline Q1217 & $12: 19: 33$ & $+49: 40: 46$ & $6.9 \times 5.1$ & 93 & $242 / 90$ & $2.19 / 2.19$ & $0.101 / 0.109$ \\
\hline GOODS-N ${ }^{e}$ & $12: 36: 52$ & $+62: 14: 20$ & $14.3 \times 10.4$ & 249 & $590 / 209$ & $2.27 / 2.31$ & $0.108 / 0.142$ \\
\hline Q1307 & $13: 07: 54$ & $+29: 22: 24$ & $10.0 \times 11.0$ & 71 & 93/- & $2.13 /-$ & $0.113 /-$ \\
\hline GWS & $14: 17: 47$ & $+52: 28: 49$ & $15.1 \times 14.8$ & 228 & $270 / 12$ & $2.82 / 2.92$ & $0.081 / 0.084$ \\
\hline Q1442 & $14: 44: 54$ & $+29: 19: 00$ & $7.3 \times 5.1$ & 137 & $613 / 373$ & $2.29 / 2.34$ & $0.124 / 0.122$ \\
\hline Q1549 & $15: 51: 55$ & $+19: 10: 53$ & $7.1 \times 5.2$ & 144 & $605 / 297$ & $2.36 / 2.29$ & $0.111 / 0.131$ \\
\hline Q1603 & $16: 04: 57$ & $+38: 11: 50$ & $7.2 \times 5.4$ & 112 & $354 / 166$ & $2.27 / 2.28$ & $0.083 / 0.080$ \\
\hline Q1623 & $16: 25: 52$ & $+26: 47: 58$ & $16.1 \times 11.6$ & 284 & $781 / 239$ & $2.18 / 2.24$ & $0.108 / 0.104$ \\
\hline Q1700 & 17:01:06 & $+64: 12: 02$ & $11.5 \times 11.0$ & 210 & $585 / 337$ & $2.29 / 2.29$ & $0.103 / 0.103$ \\
\hline Q2206 & $22: 08: 54$ & $-19: 43: 35$ & $7.5 \times 5.4$ & 119 & $457 / 183$ & $2.15 / 2.16$ & $0.113 / 0.116$ \\
\hline Q2343 & $23: 46: 20$ & $+12: 47: 28$ & $11.5 \times 6.3$ & 224 & $859 / 610$ & $2.17 / 2.17$ & $0.096 / 0.103$ \\
\hline Q2346 & $23: 48: 31$ & $+00: 22: 42$ & $11.8 \times 10.3$ & 44 & $43 / 7$ & $2.07 / 2.03$ & $0.067 / 0.070$ \\
\hline All & & & 1447 & 2862 & $9351 / 4741$ & $2.23 / 2.22$ & $0.106 / 0.113$ \\
\hline
\end{tabular}

\footnotetext{
${ }^{a}$ Mean coordinates for galaxies with spectroscopic redshifts $z>1.9$.

${ }^{b}$ Angular size of field over which spectroscopy was performed; (long axis $\times$ short axis, both in arcmin).

${ }^{c}$ The number of distinct foreground/background galaxy pairs with $D_{\text {tran }}<500 \mathrm{pkpc}$. Numbers for other ranges of $D_{\text {tran }}$ can be estimated based on Fig. 3.

${ }^{d}$ The KGPS-Full sample and the KGPS- $z_{\text {neb }}$ subsample. See Section 2.2 for definitions.

${ }^{e}$ Full catalogue published in Reddy et al. (2006); the majority of nebular redshifts in the GOODS-N region are as reported by the MOSDEF survey (Kriek et al. 2015).
}

galaxy spectra is much smaller (and less quantitative) than could be measured from high resolution, high-SNR QSO spectra. However, using galaxy-galaxy pairs provides much more rapid convergence to the mean CGM absorption as a function of impact parameter, where samples of QSO-galaxy pairs would be limited by sample variance. This improvement - along with the larger sample size - allows us to probe more details in the kinematics and the spatial distribution of $\mathrm{H}$ I compared to that obtained from QSO sightlines (e.g.Ryan-Weber 2006; Rudie et al. 2012a; Tummuangpak et al. 2014; Turner et al. 2014; Bielby et al. 2017; Tejos et al. 2014 at lower redshifts).

This paper is organized as follows. In Section 2, we describe the KBSS galaxy spectroscopic sample and the steps used in the analysis; Section 3 presents the principal results of the analysis. We discuss the implications of the results in Section 4. Particularly, in Section 4.1, we compare the results with cosmological zoom-in simulations, and in Section 4.2, we develop a simple analytic model to describe the 2D spatial and kinematic distribution of $\mathrm{HI}$ on scales $0.020-4.0 \mathrm{pMpc}$ $(\simeq 0.06-12.0 \mathrm{cMpc})$ surrounding typical SF galaxies at $z \sim 2$. We summarize our conclusions in Section 5.

Unless stated otherwise, throughout the paper we assume a $\Lambda$ CDM cosmology with $\Omega_{\mathrm{m}}=0.3, \Omega_{\Lambda}=0.7$, and $h=0.7$. Units of distance are generally given in terms of physical kpc (pkpc) or physical Mpc (pMpc).

\section{SAMPLE AND ANALYSIS}

Table 1 provides a summary of the KBSS galaxy pairs sample, described in more detail in the remainder of this section.

The KBSS galaxy pairs sample (hereafter KGPS) is drawn from 2862 galaxies in 19 densely sampled survey regions (Table 1), of which 15 comprise the nominal KBSS survey (Rudie et al. 2012a;
Steidel et al. 2014) of bright QSO sightlines. KGPS includes 4 additional fields (GOODS-N, Q1307, GWS, and Q2346) observed using the same selection criteria and instrumental configurations as the KBSS fields, and thus have a similar redshift selection function and similarly dense spectroscopic sampling. GWS and GOODS-N ${ }^{3}$ were observed as part of a Lyman break galaxy (LBG) survey targeting primarily the redshift range $2.7 \lesssim z \lesssim 3.4$ (Steidel et al. 2003), but were subsequently supplemented by observations favouring the slightly lower redshift range $1.9 \lesssim z \lesssim 2.7$ selected using a different set of rest-UV colour criteria. For all 19 fields, two groups of photometric pre-selection of candidates are included: at $z \sim 3.0 \pm 0.4$, using the MD, C, D, and M criteria described by Steidel et al. (2003); and at $z \sim 2.3 \pm 0.4$, using the BX and BM criteria described by Adelberger et al. (2004) and Steidel et al. (2004), as well as the RK criteria from Strom et al. (2017). The limiting apparent magnitude of the photometric selection is $\mathcal{R} \leq 25.5$ (AB). The galaxies were observed spectroscopically over the period 20022016, with the goal of achieving the densest possible sampling of galaxies in the redshift range $2 \lesssim z \lesssim 3$.

The survey regions listed in Table 1 are identical to those included in the analysis of galaxy-galaxy pairs by Steidel et al. (2010); however, the current spectroscopic catalogue is larger by $\sim 30$ per cent in terms of the number of galaxies with spectroscopic redshifts in the most useful range $(1.9 \lesssim z \lesssim 3.0)$, increasing the number of pairs sampling angular scales of interest by $\gtrsim 70$ per cent. More importantly, as detailed in Section 2.2 below, $\sim 50$ percent of the foreground galaxies in KGPS pairs have precisely measured systemic redshifts $\left(z_{\text {neb }}\right)$ from nebular emission lines observed in

\footnotetext{
${ }^{3}$ Referred to as 'Westphal' and 'HDF-N' by Steidel et al. (2003).
} 
the near-IR, compared with only a handful available for the S2010 analysis.

We use the substantial subset of galaxies with nebular emissionline measurements to improve the calibrations of systemic redshifts inferred from measurements of spectral features in the rest-frame UV (observed frame optical) spectra (Section 2.2). The much improved redshift precision and accuracy ${ }^{4}$ - as well as a more careful construction of composite (stacked) spectra (Section 2.4) - allow us to extend the technique using galaxy foreground-background pairs to angular separations far beyond the $\theta=15 \operatorname{arcsec}\left(D_{\text {tran }} \simeq 125\right.$ pkpc) used by Steidel et al. (2010). The various improvements represented by KGPS significantly increase the sampling density and SNR of the H I absorption measurements as a function of impact parameter $\left(D_{\text {tran }}\right)$. As we show in the next section, this leads to a major improvement compared to S2010, allowing us to resolve and model details of the kinematic structure of the $\mathrm{H}$ I with respect to the galaxies.

Subsets of the KGPS sample have figured prominently in many previous investigations involving galaxies and the CGM/IGM at 1.9 $\lesssim z \lesssim 3.5$. In what follows below, we direct the reader to the most relevant references for more information on some of the details. All of the rest-UV spectra were obtained using the Low Resolution Imaging Spectrograph (LRIS; Oke et al. 1995) on the Keck I telescope; the vast majority were obtained after 2002 June, when LRIS was upgraded to a dual-channel configuration (see Steidel et al. 2004).

A small subset of the $z \sim 2-2.6$ galaxies in earlier catalogs in some of the fields listed in Table 1 was observed in the near-IR using Keck/NIRSPEC (Erb et al. 2006a, b, c); the sample was used to calibrate UV measurements of systemic redshifts by Steidel et al. (2010). However, the vast majority of the nebular redshifts used in this paper were obtained using the Multi-Object Spectrometer for InfraRed Exploration (MOSFIRE; McLean et al. 2012; Steidel et al. 2014) beginning in 2012 April. MOSFIRE observations in all but the GOODS-N field ${ }^{5}$ were obtained as part of KBSS-MOSFIRE (see Steidel et al. 2014; Strom et al. 2017 for details.)

The statistical properties of the galaxies in the KGPS sample are as described in previous work: stellar masses $8.6 \lesssim \log \left(M_{*} / \mathrm{M}_{\odot}\right) \lesssim$ 11.4 (median $\simeq 10.0)$, star formation rates $2 \lesssim \mathrm{SFR} /\left(\mathrm{M}_{\odot} \mathrm{yr}^{-1}\right) \lesssim$ 300 (median $\simeq 25$ ) (Shapley et al. 2005; Erb et al. 2006a, b; Reddy et al. 2008, 2012; Steidel et al. 2014; Strom et al. 2017; Theios et al. 2019), and clustering properties indicate host dark matter haloes of typical mass $\left\langle\log \left(M_{\mathrm{h}} / \mathrm{M}_{\odot}\right)\right\rangle=11.9 \pm 0.1$ (Adelberger et al. 2005a; Trainor \& Steidel 2012).

\subsection{Rest-frame far-UV spectra}

All of the rest-UV spectra used in this work were obtained with the Low Resolution Imaging Spectrometer (LRIS; Oke et al. 1995; Steidel et al. 2004) on the Keck I 10-m telescope; most were obtained between 2002 and 2016, after LRIS was upgraded to a dual-beam spectrograph. Most of the spectra used here were obtained using the blue channel (LRIS-B), with one of two configurations: a 400 line/mm grism blazed at $3400 \AA$ in first order, covering 3200-6000 A, or a 600 line/mm grism blazed at $4000 \AA$, typically covering 3400 $5600 \AA$. Approximately half of the slitmasks were observed with each

\footnotetext{
${ }^{4}$ The number of galaxies with insecure or incorrect redshifts is also greatly reduced compared to $\mathrm{S} 2010$.

${ }^{5}$ Nebular redshifts of 89 galaxies in our catalogue were obtained by the MOSFIRE Deep Evolution Field Survey (MOSDEF; Kriek et al. 2015.)
}

configuration. Further details on the observations and reductions with LRIS-B are given in, e.g. Steidel et al. (2004, 2010, 2018).

The total integration time for individual objects ranges from $5400 \mathrm{~s}$ to $>54000$ s. About 40 per cent of the galaxies were observed with two or more masks, particularly in the KBSS fields for which the field size is comparable to the 5.5 by 7.5 field of view of LRIS. Examples of typical reduced 1D spectra are shown in Fig. 1. The wavelength solutions for the LRIS-B spectra were based on polynomial fits to arc line lamp observations using the same mask and instrument configuration, which have typical residuals of $\simeq 0.1 \AA$. Small shifts between the arc line observations and each 1800 s science exposure were removed during the reduction process with reference to night sky emission features in each science frame. The wavelength calibration uncertainties make a negligible contribution to the redshift measurement errors (Section 2.2).

Each reduced 1D spectrum ${ }^{6}$ was examined interactively, in order to mask regions of very low SNR, poor background subtraction, unphysical flux calibration, or previously unmasked artefacts (e.g. cosmic rays, bad pixels) that were not recognized during data reduction. A total of 280 spectra (out of nearly 10000 in total) were entirely discarded because of generally poor quality or unphysical continuum shape. Spectra of the same object observed on multiple masks and/or with multiple spectroscopic setups were assigned individual weights according to spectral quality, based on a combination of visual inspection and exposure time. They were then resampled on to a common wavelength grid with the finest sampling of the individual spectra to preserve the spectral resolution (using cubic-spline interpolation) and averaged together to create a single spectrum for each object.

\subsection{Calibration of systemic redshifts}

Spectral features commonly observed in the far-UV spectra of high redshift SF galaxies - Ly $\alpha$ emission, when present, and interstellar (IS) absorption from strong resonance lines of (e.g.) Si II, Si IV, C II, C IV, O I - are rarely found at rest with respect to the stars in the same galaxy due to gas motions and radiative transfer effects (e.g. Steidel et al. 1996; Franx et al. 1997; Lowenthal et al. 1997; Pettini et al. 2001; Shapley et al. 2003; Erb et al. 2006b). Clearly, measuring the kinematics of diffuse gas in the CGM of foreground galaxies benefits from the most accurate available measurements of each galaxy's systemic redshift $\left(z_{\text {sys }}\right)$.

The centroids of nebular emission lines from ionized gas (i.e. H II regions) are less strongly affected by galaxy-scale outflows and radiative transfer effects, and are generally measured with significantly higher precision, than the rest-FUV features. As previously noted, 50 per cent of the foreground galaxies used in this work have measurements of one or more strong nebular emission lines in the rest-frame optical (observed frame $J, H, K$ bands) using MOSFIRE. Independent observations of the same galaxies with MOSFIRE have demonstrated redshift precision (i.e. rms repeatability) of $\sigma_{v} \simeq$ $18 \mathrm{~km} \mathrm{~s}^{-1}$ (Steidel et al. 2014).

For the 50 percent of foreground galaxies lacking nebular emission-line measurements, we used estimates of $z_{\text {sys }}$ based on the full KBSS-MOSFIRE sample (Steidel et al. 2014; Strom et al. 2017) with $z_{\text {neb }}>1.9$ and existing rest-UV LRIS spectra. These were used to calibrate relationships between $z_{\text {sys }}$ and redshifts measured from features in the rest-frame FUV spectra, strong interstellar

${ }^{6}$ For galaxies observed on multiple masks, each independent 1D spectrum was examined separately. 

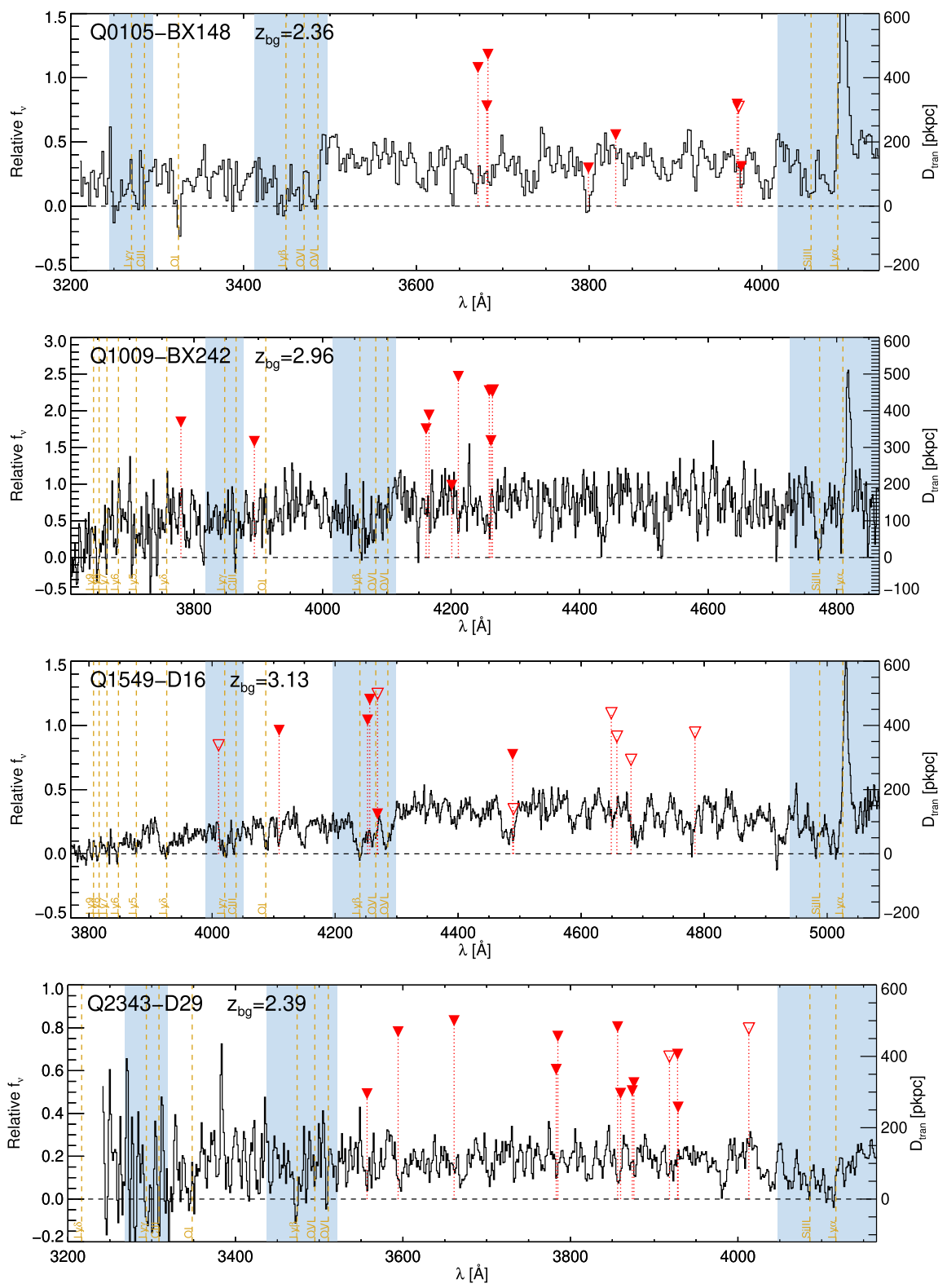

Figure 1. Randomly selected examples of individual rest-UV spectra of background galaxies in KGPS. Wavelengths are in the observed frame, with red triangles marking the position of Ly $\alpha \lambda 1215.67$ at wavelengths $1215.67\left(1+z_{\mathrm{fg}}\right) \AA$ for each foreground galaxy with projected distance $D_{\text {tran }} \leq 500$ pkpc. The solid (open) triangles correspond to foreground galaxies whose systemic redshifts are based on $z_{\text {neb }}\left(z_{\mathrm{UV}}\right)$. Their typical redshift uncertainties, in terms of observed wavelength, are $\sim 0.2 \AA(1.8 \AA)$ at $z=2.2$ as estimated in Section 2.2. The $y$-coordinate of each triangle indicates $D_{\text {tran }}$ for the foreground galaxies, with reference to the scale marked on the right-hand side of each plot. The light shaded regions are those that would be masked prior to using the spectrum to form composites stacked in the rest frame of foreground galaxies (see Section 2.4 and Table 2), to minimize contamination by spectral features at $z=z_{\mathrm{bg}}$. The yellow vertical lines indicate UV absorption lines arising in the ISM of the background galaxy. Note that some foreground galaxies have clear counterparts in the Ly $\alpha$ forest, even in low-resolution spectra.

absorption lines $\left(z_{\mathrm{IS}}\right)$ and/or the centroid of Lyman $\alpha$ emission $\left(z_{\mathrm{Ly} \alpha}\right)$. As for previous estimates of this kind (e.g. Adelberger et al. 2003; Steidel et al. 2010; Rudie et al. 2012a), we adopt rules that depend on the particular combination of features available in each spectrum. Fig. 2 shows the distribution of velocity offsets of the UV redshift measurements relative to $z_{\mathrm{neb}}, \Delta v_{\mathrm{Ly} \alpha}=c\left(z_{\mathrm{Ly} \alpha}\right.$ $\left.-z_{\text {neb }}\right) /\left(1+z_{\text {neb }}\right)$ and/or $\Delta v_{\text {IS }}=c\left(z_{\text {IS }}-z_{\text {neb }}\right) /\left(1+z_{\text {neb }}\right)$ for the three cases below. The median velocity offsets (see Fig. 2) for the three sub-samples were then used to derive the following relationships that map UV redshift measurements to an estimate of $z_{\text {sys }}$ (i) Case 1: $z_{\mathrm{Ly} \alpha}$ only,

$z_{\mathrm{sys}}=z_{\mathrm{Ly} \alpha}-\frac{237 \mathrm{~km} \mathrm{~s}^{-1}}{c}\left(1+z_{\mathrm{Ly} \alpha}\right)$,

(ii) Case 2: $z_{\text {IS }}$ only,

$z_{\mathrm{sys}}=z_{\mathrm{IS}}+\frac{97 \mathrm{~km} \mathrm{~s}^{-1}}{c}\left(1+z_{\mathrm{IS}}\right)$.

(iii) Case 3: $z_{\text {Ly } \alpha}$ and $z_{\text {IS }}$, 


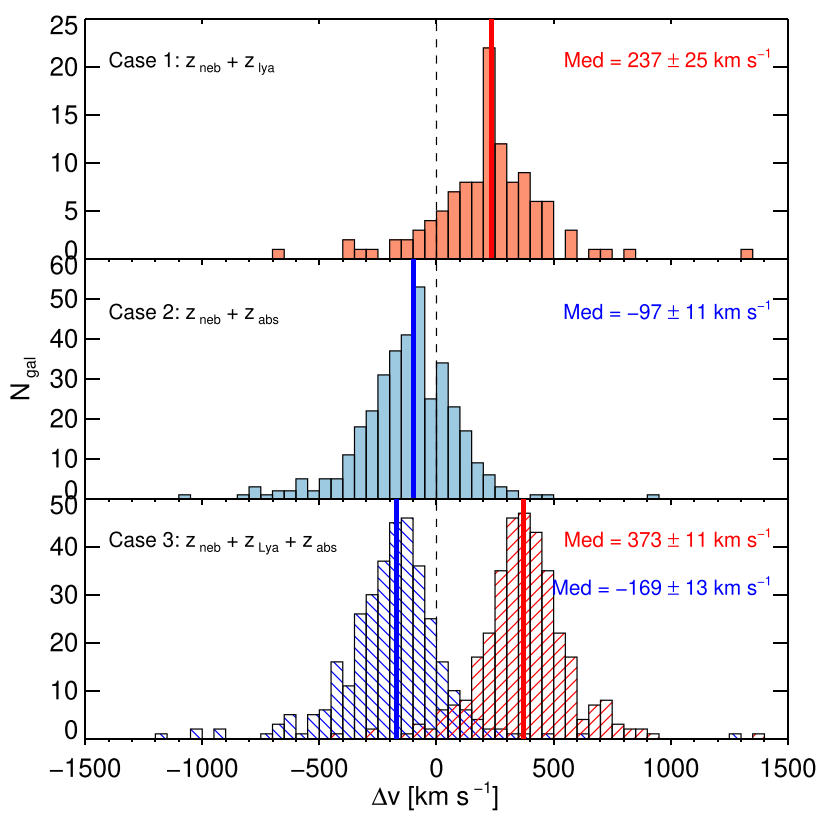

Figure 2. Distribution of velocity differences between $z_{\mathrm{Ly} \alpha}\left(\Delta v_{\mathrm{Ly} \alpha}\right.$, in red) or $z_{\text {IS }}\left(\Delta v_{\text {IS }}\right.$, in blue) and the systemic redshift measured from $z_{\text {neb }}$. Top-right of each panel shows the median shift in velocity and its error estimated by dividing the standard deviation by the square root of the number of galaxies. These distributions have been used to calibrate the systemic redshifts of galaxies in equations (1)-(3), for which only the UV spectral features are available.

$$
\begin{aligned}
\text { if } z_{\mathrm{Ly} \alpha} & >z_{\mathrm{IS}}, \\
z_{\mathrm{sys}}= & \frac{1}{2}\left\{\left[z_{\mathrm{Ly} \alpha}-\frac{373 \mathrm{~km} \mathrm{~s}^{-1}}{c}\left(1+z_{\mathrm{Ly} \alpha}\right)\right]\right. \\
& \left.+\left[z_{\mathrm{IS}}+\frac{169 \mathrm{~km} \mathrm{~s}^{-1}}{c}\left(1+z_{\mathrm{IS}}\right)\right]\right\} ;
\end{aligned}
$$

otherwise,

$z_{\mathrm{sys}}=\frac{1}{2}\left(z_{\mathrm{Ly} \alpha}+z_{\mathrm{abs}}\right)$.

When the above relations are used to estimate $z_{\text {sys, uv }}$ on an object by object basis, applied to the UV measurements of the redshift calibration sample on an object-by-object basis, the outlier-clipped mean and rms of the velocity difference between $z_{\text {neb }}$ and $z_{\text {sys, uv }}$ are

$\left\langle c\left(z_{\text {sys,uv }}-z_{\text {neb }}\right) /\left(1+z_{\text {neb }}\right)\right\rangle=-5 \pm 143 \mathrm{~km} \mathrm{~s}^{-1}$,

implying that we expect negligible systematic offset in cases where only $z_{\text {sys, uv }}$ is available, with $\sigma_{\mathrm{z}} \simeq 140 \mathrm{~km} \mathrm{~s}^{-1}$.

In what follows below, we define the subsample of galaxy foreground-background pairs for which $z_{\text {sys, fg }}$ is based on $z_{\text {neb }}$ as the 'KGPS- $z_{\text {neb' }}$ ' sample, with $\sigma_{z} \simeq 18 \mathrm{~km} \mathrm{~s}^{-1}$; the 'KGPS-Full' sample includes all of KGPS- $z_{\text {neb }}$ plus the remaining pairs for which $z_{\text {sys, fg }}$ is estimated from the rest-UV spectra according to equations (1)-(3). The fraction of pairs with $z_{\text {sys, fg }}$ estimated from rest-UV features is consistently $\simeq 50$ per cent at $D_{\text {tran }}<1 \mathrm{pMpc}$, but gradually decreases to $\simeq 40$ per cent from $D_{\text {tran }} \simeq 1 \mathrm{pMpc}$ to $4 \mathrm{pMpc}$.

\subsection{Assembly of galaxy foreground-background pairs}

We assembled samples of KGPS galaxy pairs $\left(z_{\mathrm{fg}}, z_{\mathrm{bg}}\right)$ according to several criteria designed to optimize the measurement of weak ab- sorption lines near the redshift $z_{\mathrm{fg}}$ in the spectrum of the background galaxy:

(i) The background galaxy has one or more LRIS spectra.

(ii) The foreground galaxy does not host a known type I or type II active galactic nucleus. ${ }^{7}$

(iii) The paired galaxies have redshifts $\left(z_{\mathrm{fg}}, z_{\mathrm{bg}}\right)$ such that

$0.017\left(1+z_{\mathrm{fg}}\right)<\Delta z_{\mathrm{fb}}<0.3\left(1+z_{\mathrm{fg}}\right)$,

where $\Delta z_{\mathrm{fb}}=z_{\mathrm{bg}}-z_{\mathrm{fg}}$. This is equivalent to $5100 \mathrm{~km} \mathrm{~s}^{-1}<\Delta v_{\mathrm{LOS}}$ $<90000 \mathrm{~km} \mathrm{~s}^{-1}$, or $37 \mathrm{pMpc}<D_{\mathrm{LOS}}<520 \mathrm{pMpc}$ at $z=2.2$, assuming pure Hubble flow, where $\Delta v_{\mathrm{LOS}}$ and $D_{\mathrm{LOS}}$ are velocity difference and distance in the line-of-sight direction.

The lower limit on $\Delta z_{\mathrm{fb}}$ ensures that the two galaxies are not physically associated, so that any absorption features detected near $z_{\text {fg }}$ are well-separated from features at $z_{\text {bg }}$ and are not part of the same large-scale structure in which the background galaxy resides. An upper limit on $\Delta z_{\mathrm{fb}} /\left(1+z_{\mathrm{fg}}\right)$ was set by $\mathrm{S} 2010$ to maximize the detectability of C IV $\lambda \lambda 1548,1550$ at $z_{\mathrm{fg}}$ by ensuring that it would fall longward of the Ly $\alpha$ forest in the spectrum of the background galaxy, i.e.

$\left(1+z_{\text {fg }}\right) 1549 \AA>\left(1+z_{\text {bg }}\right) 1215.67 \AA$

for typical $z_{\mathrm{bg}} \sim 2.4$. In this case, using a more empirical approach, we tested different upper limits on $\Delta z_{\mathrm{fb}} /\left(1+z_{\mathrm{fg}}\right)$ in order to optimize the SNR of the final stacks. In principle, if one is interested in detecting Ly $\alpha$ absorption, large $\Delta z_{\mathrm{fb}} /\left(1+z_{\mathrm{fg}}\right)$ would increase the relative contribution of the shorter wavelength, noisier portions of the background galaxy spectra, particularly when regions shortward of $\operatorname{Ly} \beta$ at $z=z_{\mathrm{bg}}\left[\right.$ i.e. $\left.\lambda \leq\left(1+z_{\mathrm{bg}}\right) 1025.7 \AA\right]$ are included. On the other hand, choosing a small upper limit on $\Delta z_{\mathrm{fb}} /\left(1+z_{\mathrm{fg}}\right)$ would increase the noise by significantly decreasing the number of spectra contributing. Depending on the strength of the Ly $\alpha$ absorption, we found that the SNR does not depend strongly on the upper limit so long as it is close to $\Delta z_{\mathrm{fb}} /\left(1+z_{\mathrm{fg}}\right) \simeq 0.30$, similar to the upper limit used by Steidel et al. (2010) $\left(\Delta z_{\mathrm{fb}} /\left(1+z_{\mathrm{fg}}\right) \simeq 0.294\right.$ for $\left.z_{\mathrm{bg}} \simeq 2.4\right)$.

Fig. 3 shows the cumulative number of galaxy pairs as a function of $D_{\text {tran }}$ between the foreground galaxy and the line of sight to the background galaxy, evaluated at $z_{\mathrm{fg}}$. The cumulative number of distinct pairs varies as $D_{\text {tran }}^{2}$ (dashed lines in Fig. 3; as expected for uniform sampling of a constant surface density of galaxies) over the range $50 \leq D_{\text {tran }} / \mathrm{pkpc} \leq 500$. The departure of the observed number of pairs falls below the quadratic extrapolation for $D_{\text {tran }}<30 \mathrm{pkpc}$ (angular scales of $\theta \lesssim 3$ "' 6 at $z \sim 2.2$ ) due to a combination of limited spatial resolution of the ground-based images used to select targets, and the constraints imposed by slit assignment on LRIS slitmasks. For $D_{\text {tran }} \gtrsim 1 \mathrm{pMpc}(\theta \gtrsim 2 \operatorname{arcmin}$ at $z=2.2)$, the number of pairs begins to be limited by the size of individual survey regions (see Table 1). Over the range $30<D_{\text {tran }} / \mathrm{pkpc}<1000$, the number of pairs is well represented by a quadratic function

KGPS-Full: $\quad N_{\text {pair }}\left(<D_{\text {tran }}\right)=39069 \times\left(\frac{D_{\text {tran }}}{1 \mathrm{pMpc}}\right)^{2}$,

and

KGPS $-z_{\text {neb }}: \quad N_{\text {pair }}\left(<D_{\text {tran }}\right)=19577 \times\left(\frac{D_{\text {tran }}}{1 \mathrm{pMpc}}\right)^{2}$.

${ }^{7}$ Pairs for which the foreground object harbours an AGN will be considered in a separate paper. 


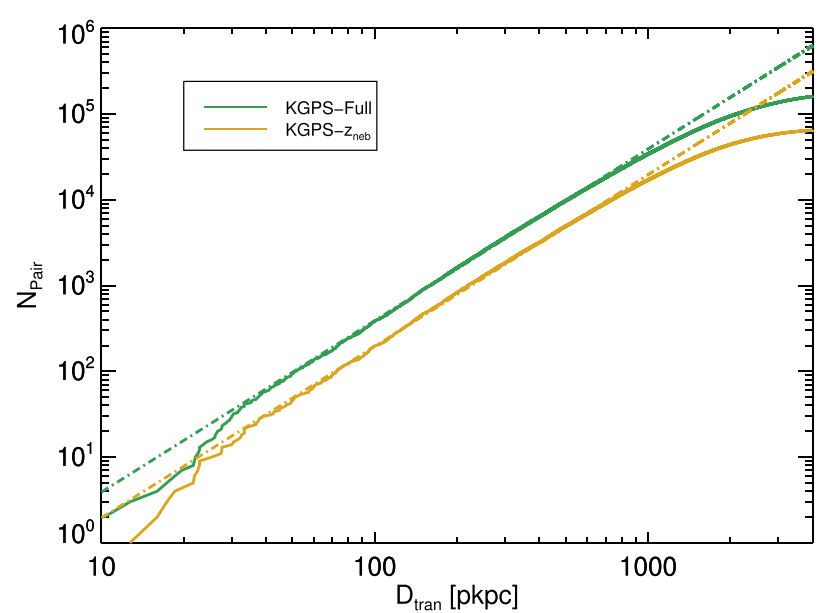

Figure 3. The cumulative number of pairs as a function of impact parameter $D_{\text {tran }}\left(z_{\mathrm{fg}}\right)$ for the KGPS-Full sample (green), the KGPS- $z_{\text {neb }}$ sample (yellow). The solid curves are the actual number of pairs, and the dash-dotted lines are quadratic fits over the range $50 \mathrm{pkpc}<D_{\text {tran }}<500 \mathrm{pkpc}$ for each of the KGPS samples. The smaller number of pairs (relative to the quadratic extrapolation) at very small $D_{\text {tran }}$ results from observational biases caused by geometrical slitmask constraints and finite angular resolution of the groundbased images used for target selection. At large $D_{\text {tran }}$, the pair count falls below the quadratic fit as the angular separation approaches the size of the KBSS survey regions.

Fig. 4 shows the distributions of $z_{\mathrm{fg}}$ and $\Delta z_{\mathrm{fb}} /\left(1+z_{\mathrm{fg}}\right)$ for both the KBSS-Full and KBSS- $z_{\text {neb }}$ samples. The difference in the $z_{\mathrm{fg}}$ distributions is caused by gaps in redshift for the KBSS- $z_{\text {neb }}$ sample that correspond to regions of low atmospheric transmission between the $J, H$, and $K$ bands. However, in spite of this, the distributions of $\Delta z_{\mathrm{fb}} /\left(1+z_{\mathrm{fg}}\right)$ remain very similar. The median foreground galaxy redshift is $\left\langle z_{\mathrm{fg}}\right\rangle_{\text {med }}=2.23$ for the KGPS-Full sample, and $\left\langle z_{\mathrm{fg}}\right\rangle_{\mathrm{med}}=$ 2.22 for the KGPS- $z_{\text {neb }}$ sample; the median value of the redshift differences between the foreground and background galaxies are $\Delta z_{\mathrm{fb}} /\left(1+z_{\mathrm{fg}}\right)=0.106$ and 0.113 , respectively.

\subsection{Composite spectra}

The typical spectrum of an individual galaxy in the KGPS sample has SNR per spectral resolution element of only 1-6 in the region shortward of Ly $\alpha$ in the galaxy rest frame. Individual spectra also include absorption from other spectral lines due to the interstellar and circumgalactic medium of the galaxy itself - whose locations are predictable - and intervening absorption caused by gas at redshifts different from the foreground at which a measurement of Ly $\alpha$ absorption is made. ${ }^{8}$ Both problems - limited SNR of faint background galaxy spectra, and contamination from absorption at other redshifts - are mitigated by forming 'stacks' of many spectra sampling a particular range of $D_{\text {tran }}$ for an ensemble of foreground galaxies probed by background galaxies.

A distinct advantage of stacking, particularly when it comes to detecting absorption lines arising from gas at a particular redshift, is that one naturally suppresses small-wavelength-scale noise caused by contamination from absorption lines at redshifts other than that of the foreground galaxies of interest. With a suitable number of spectra

\footnotetext{
${ }^{8}$ In the case of QSO sightlines, the problem of contamination also exists, but is partially overcome by observing at very high spectral resolution.
}
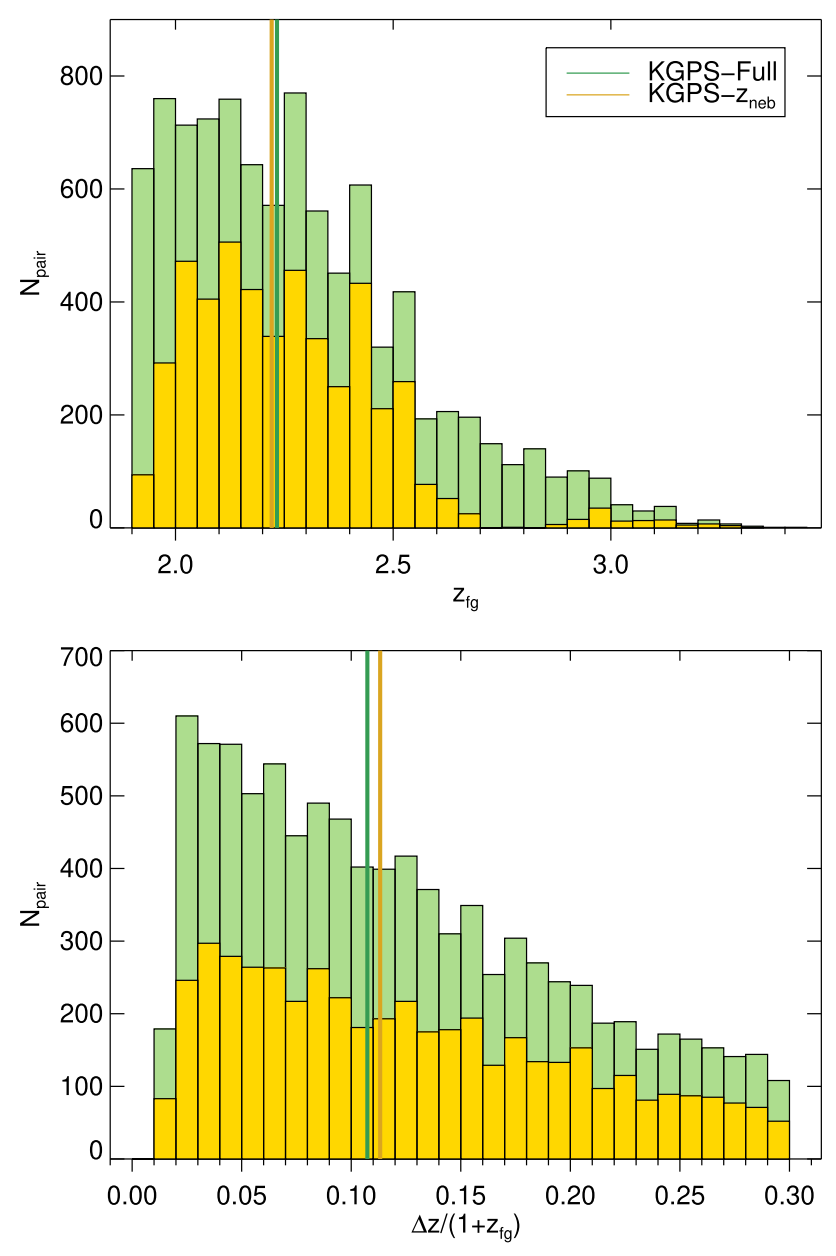

Figure 4. Distribution of $z_{\mathrm{fg}}$ (top) and $\Delta z_{\mathrm{fb}} /\left(1+z_{\mathrm{fg}}\right)$ (bottom) for foreground-background galaxy pairs with with $D_{\text {tran }}<4.7 \mathrm{pMpc}$. The vertical lines mark the median values of each distribution. The KGPS-Full and KGPS$z_{\text {neb }}$ samples have similar distributions of both $z_{\mathrm{fg}}$ and $\Delta z_{\mathrm{fb}} /\left(1+z_{\mathrm{fg}}\right)$.

comprising a stack, unrelated (stochastic) absorption will produce a new, lower, effective continuum level against which the Ly $\alpha$ absorption due to the foreground galaxy ensemble can be measured. The amount by which the continuum is lowered depends on $z_{\mathrm{fg}}$, and is expected to be close to the mean Ly $\alpha$ forest flux decrement $D_{\mathrm{A}}(z)$ (Oke \& Korycansky 1982). At $z \sim 2-2.5$ most relevant for the KGPS sample, $D_{\mathrm{A}} \simeq 0.2$, i.e. a reduction in the apparent continuum level near Ly $\alpha$ of $\simeq 20$ per cent. Thus, any residual Ly $\alpha$ absorption is equivalent to an HI 'overdensity', in the sense that it signals the amount by which HI gas associated with the foreground galaxy exceeds the mean IGM absorption at the same redshift.

We arranged galaxy pairs into bins of $D_{\text {tran }}$, and stacked the spectra of all of the background galaxies in the same bin of $D_{\text {tran }}$ after (1) normalizing the flux-calibrated background galaxy spectra to have unity median flux density evaluated over the $z_{\text {bg }}$-frame rest wavelength interval $1300 \leq \lambda_{\mathrm{bg}, 0} / \AA \leq 1400$; (2) masking regions of the background galaxy spectra corresponding to the locations of strong absorption lines or sets of absorption lines at $z=z_{\text {bg }}$; the relevant spectral ranges are given in Table 2; (3) shifting the result to the rest frame of the foreground galaxy, i.e.

$\lambda_{\mathrm{fg}, 0}=\lambda_{\mathrm{bg}, \mathrm{obs}} /\left(1+z_{\mathrm{fg}}\right)$ 
Table 2. Masked background spectral regions.

\begin{tabular}{lcc}
\hline$\lambda(\AA)$ & $\Delta z_{\mathrm{fb}} /\left(1+z_{\mathrm{fg}}\right)$ & Spectral features \\
\hline $965-980$ & $0.2405-0.2598$ & Ly $\gamma$, C III $\lambda 977$ \\
$1015-1040$ & $0.1689-0.1977$ & Ly $\beta$, O VI $\lambda 1031,1036$ \\
$1195-1216$ & $0-0.0173$ & Si III $\lambda 1206$, Ly $\alpha$ \\
\hline
\end{tabular}

and (4) resampling the normalized, masked, and $z_{\mathrm{fg}}$-shifted spectra on to a common range of rest wavelength and combining to form a composite rest-frame spectrum representing the bin in $D_{\text {tran }}$.

The normalization in (1) was performed in order to give roughly equal weight to each galaxy pair in a given bin of $D_{\text {tran }}$ without requiring an actual continuum fit to each low-SNR spectrum. We found that continuum fitting is less prone to large systematic errors (e.g. Faucher-Giguère et al. 2008) when it is performed for the composite spectra after stacking, rather than for individual spectra, particularly within the $\operatorname{Ly} \alpha$ forest where it would be difficult to perform the fit reliably in the face of both shot noise and real Ly $\alpha$ forest absorption. Error spectra for each stack were generated using bootstrap resampling of the galaxy ensemble, which should account for both sample variance and random (Poisson) errors. Step (2) was implemented in order to reduce contamination by unrelated absorption features near $z_{\text {bg }}$ by excluding pixels known to be contaminated; the wavelength ranges listed in Table 2 were adopted based on tests using larger or smaller masked intervals. ${ }^{9}$ For step (3), we used the best available systemic redshift of the foreground galaxy (Section 2.2).

We experimented with several different methods for accomplishing step (4) before adopting a straight median of unmasked pixels at each dispersion point; details of the tests are summarized in Appendix A. The median algorithm produces composite spectra with SNR comparable to the best $\sigma$-clipped mean algorithm, but has the added benefit of computational and conceptual simplicity.

To remove the continuum, each composite spectrum was divided into 200- $\AA$ segments; for each segment, we calculated the mean value (with $2.5 \sigma$ clipping applied) of the flux density; the mean flux density and mean wavelength within each segment were then used to constrain a cubic spline fit to the continuum flux density as a function of wavelength, as illustrated in Fig. 5. The composite spectra were then divided by the initial continuum fit, after which the continuum level of each normalized spectrum was further adjusted by dividing by the linear interpolation of pixels within two windows fixed in velocity relative to the nominal rest wavelength of Ly $\alpha: 3000 \mathrm{~km} \mathrm{~s}^{-1}$ $<\left|\Delta v_{\text {Ly } \alpha}\right|<5000 \mathrm{~km} \mathrm{~s}^{-1}$. Fig. 5 shows examples of the $z_{\mathrm{fg}}$-frame stacked spectra before and after dividing by a fitted continuum. Both the velocity width and the depth of the excess Ly $\alpha$ absorption associated with the foreground galaxies clearly varies with $D_{\text {tran }}$.

\section{H I ABSORPTION}

\subsection{Ly $\alpha$ rest equivalent width $\left[W_{\lambda}(\operatorname{Ly} \alpha)\right]$}

Expressing the total strength of $\operatorname{Ly} \alpha$ absorption in terms of the rest-frame equivalent width [hereafter $W_{\lambda}(\operatorname{Ly} \alpha)$ ] is appropriate in this case, where the velocity structure is only marginally resolved. Because $W_{\lambda}(\operatorname{Ly} \alpha)$ does not depend on the spectral resolution of the observed spectra (provided it is sufficient to allow accurate placement

\footnotetext{
${ }^{9}$ Note that masking a particular range of rest wavelength in the frame of each background galaxy is similar to eliminating pairs having particular range of $\Delta z_{\mathrm{fb}} /\left(1+z_{\mathrm{fg}}\right)$. These intervals are also provided in Table 2 .
}

of the continuum level), it is also useful for comparisons among samples obtained with different spectral resolution. $W_{\lambda}(\operatorname{Ly} \alpha)$ is also entirely empirical, and does not depend on any assumptions regarding the fine-scale kinematics or component structure of the absorbing gas.

In KGPS, which uses composite spectra of many background galaxies, $W_{\lambda}(\operatorname{Ly} \alpha)$ is modulated by a potentially complex combination of the mean integrated covering fraction of absorbing gas, its line-of-sight (LOS) kinematics for an ensemble of sightlines falling within a range of $D_{\text {tran }}$ relative to foreground galaxies, and the total column density of HI. As discussed above (see also S2010), the finite 'footprint' of the image of a background galaxy projected on to the gas distribution surrounding a foreground galaxy also means that the Ly $\alpha$ absorption profile may depend on the spatial variations on scales of a few $\mathrm{pkpc}^{10}$ for CGM sightlines near individual galaxies. However, given a sample of foreground galaxies, the average dependence of $W_{\lambda}(\operatorname{Ly} \alpha)$ on $D_{\text {tran }}$ should be identical for extended (galaxy) and point-like (QSO) background sources so long as the number of sightlines is large enough to overcome sample variance within each bin of $D_{\text {tran }}$.

For a sample of galaxy pairs as large as KGPS, where both the width and depth of the Ly $\alpha$ profile varies with $D_{\text {tran }}$ (Fig. 5), it is desirable to develop a robust method for automated measurement of $W_{\lambda}(\operatorname{Ly} \alpha)$ while maximizing the SNR. For spectra of limited continuum SNR - particularly where the absorption line profile has a spectral shape that is unknown a priori, the size of the measurement aperture has a significant effect on the SNR of the Ly $\alpha$ line; it should not be unnecessarily large, which would contribute unwanted noise without affecting the net signal, nor so small that it would exclude significant absorption signal. We set the integration aperture using 2D maps of apparent optical depth (Fig. 8; to be discussed in detail in Section 3.2): when $D_{\text {tran }}<100 \mathrm{pkpc}$, we set the aperture width to $\Delta v=1400 \mathrm{~km} \mathrm{~s}^{-1}\left(\Delta \lambda_{0} \simeq 11.35 \AA\right)$, centred on the nominal rest wavelength of $\operatorname{Ly} \alpha$; otherwise, the width of the aperture is set to

$\Delta v=1000\left\langle\log \left(D_{\operatorname{tran}} / \mathrm{pkpc}\right)\right\rangle-600 \mathrm{~km} \mathrm{~s}^{-1}$,

also centred on rest-frame $\operatorname{Ly} \alpha$.

Fig. 6 shows $W_{\lambda}(\operatorname{Ly} \alpha)$ measured from the KGPS-Full sample as a function of $D_{\text {tran }}$ with the bin size in $D_{\text {tran }}$ set to be 0.3 dex, together with measurements from S2010 for galaxy-galaxy foreground/background pairs and from Turner et al. (2014) for foreground galaxy-background QSO pairs from the KBSS survey. The points from Turner et al. (2014) were measured from the spectra of only 17 background QSOs in the 15 KBSS fields, evaluated at the redshifts of foreground galaxies within $\simeq 4.2$ drawn from essentially the same parent galaxy sample as KGPS. For $D_{\text {tran }}>$ $400 \mathrm{pkpc}$, where the sample variance of QSO-galaxy sightlines is relatively small, there is excellent agreement between KGPS-Full and Turner et al. (2014), as expected. At smaller $D_{\text {tran }}$, the QSO sightline measurements are not as detailed, although they remain statistically consistent given the larger uncertainties. Although the QSO spectra used by Turner et al. (2014) are far superior to the KGPS galaxy spectra in both resolution $\left(\sigma_{\mathrm{v}} \simeq 8 \mathrm{~km} \mathrm{~s}^{-1}\right.$ versus $\sigma_{v}$ $\left.\simeq 190 \mathrm{~km} \mathrm{~s}^{-1}\right)$ and SNR $(\simeq 100$ versus $\simeq$ a few $)$, the QSO-based measurements are less precise for the ensemble. This is because both the local continuum level and the net absorption profile contribute to the uncertainty. In the case of the QSO sightlines, the stochastic variations in the mean $\operatorname{Ly} \alpha$ forest opacity in the QSO spectra in the vicinity of $z_{\text {fg }}$ modulate the apparent continuum against which excess

\footnotetext{
${ }^{10}$ The typical effective radius of the background galaxies in the KBSS sample
} is $r_{\mathrm{e}} \simeq 1.5 \mathrm{pkpc}$ (Law et al. 2012). 

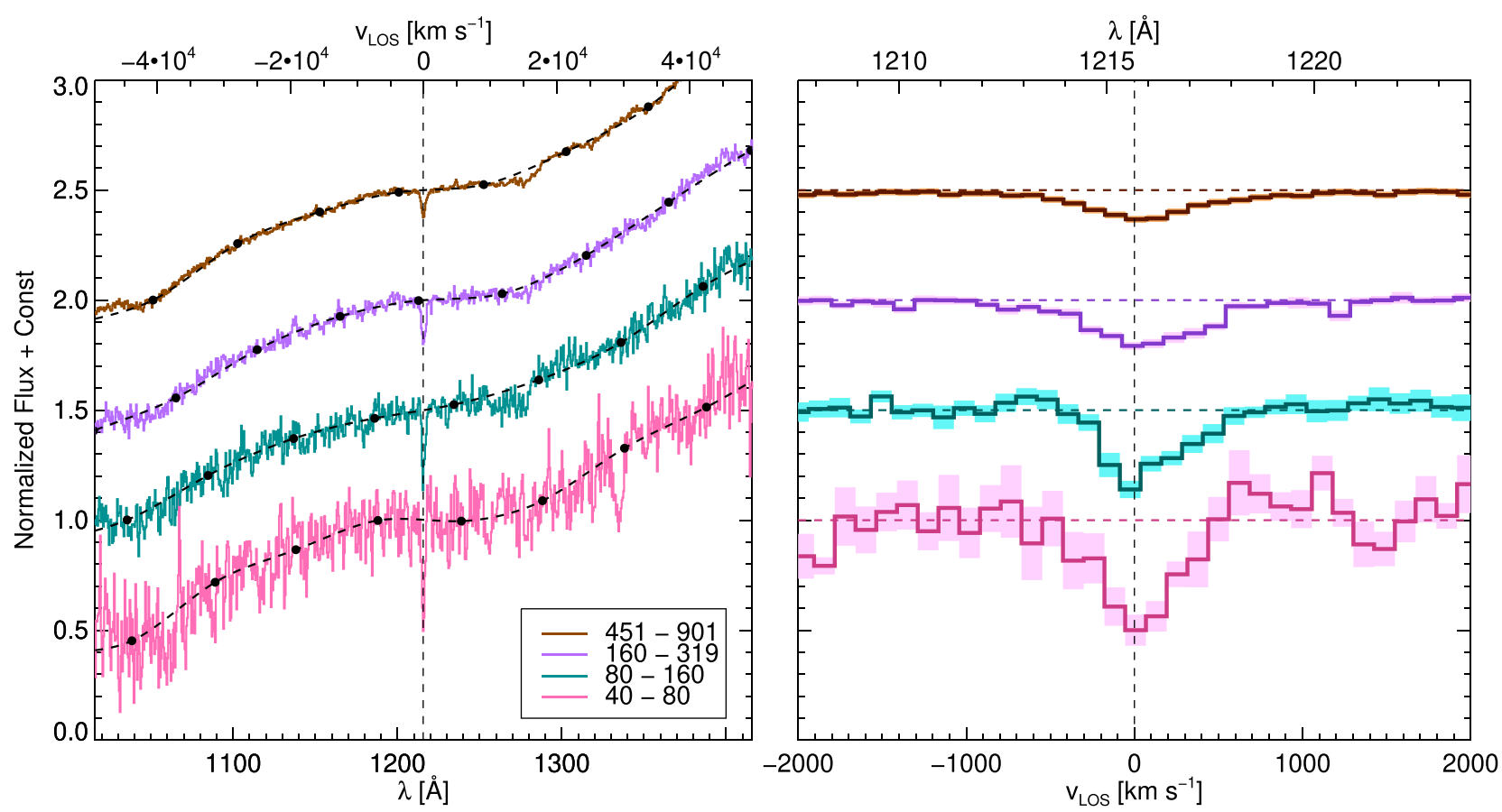

Figure 5. Example composite spectra near Ly $\alpha$ in the rest frame of foreground galaxies for four different bins of $D_{\text {tran }}$. The left-hand panel shows the spectra before continuum normalization. The black curves with dots are the fitted continua and spline points used for cubic interpolation. The legend indicates the range in pkpc for the bin in $D_{\text {tran. }}$. The right-hand panel shows normalized spectra near Ly $\alpha$, along with their $1 \sigma$ uncertainty (shaded histogram). In both panels, the spectra shown have been shifted relative to one another by 0.5 in $y$ for display purposes. The vertical dashed line is the rest wavelength of Ly $\alpha, 1215.67 \AA$. The Ly $\alpha$ absorption profiles in the composite spectra clearly vary in both depth and width with $D_{\text {tran }}$. The clear asymmetry in the velocity profiles of the two middle spectra ([50-100] and [160-319] pkpc) is discussed in Section 4.3 below.

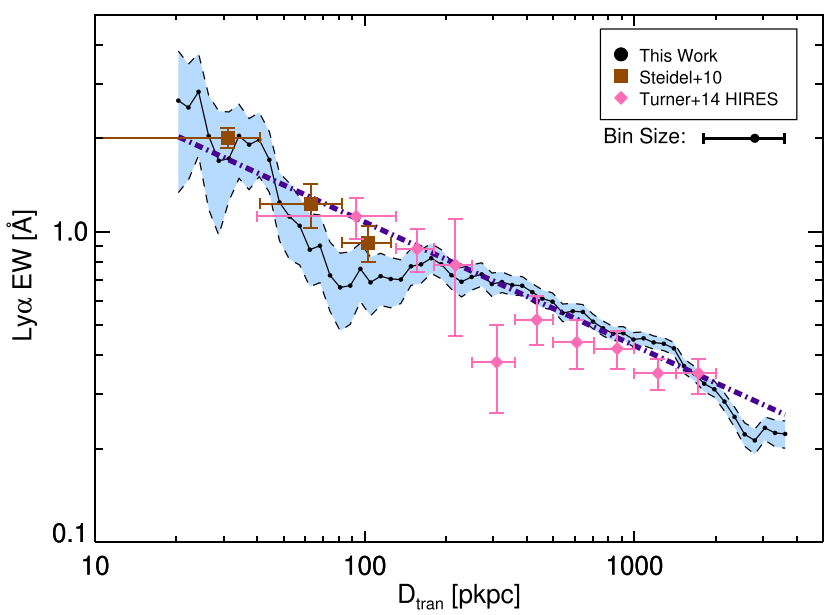

Figure 6. $W_{\lambda}(\operatorname{Ly} \alpha)$ as a function of $D_{\text {tran }}$ measured from the KGPSFull sample. Each black dot (connected with the solid line segments) is a measurement made from a composite spectrum in a bin spanning a factor of 2 in $D_{\text {tran }}$ where the point marks the geometric mean $D_{\text {tran }}$ within the bin. The light blue shaded region represents the $\pm 1 \sigma$ uncertainty based on bootstrap resampling of the spectra comprising each composite in each bin. The single dot with error bars below the legend box shows the bin size used to make composite spectra; i.e. the bins were evaluated at intervals smaller than the bin size, thus adjacent points on smaller than the bin size are correlated. The purple dash-dotted line is the best single power-law fit to the $W_{\lambda}(\operatorname{Ly} \alpha)-$ $D_{\text {tran }}$ relation, with slope $\beta=-0.40 \pm 0.01$. The new measurements are compared with Steidel et al. (2010, brown squares) and Turner et al. (2014, magenta diamonds), where the latter are based on HIRES QSO/galaxy pairs in KBSS.
Ly $\alpha$ absorption at $z_{\mathrm{fg}}$ is evaluated, and have a large sample variance in the absorption strength at fixed $D_{\text {tran }}$. The points in Fig. 6 from S2010 were measured using stacked LRIS spectra of a subset of the current KGPS sample, with a comparable range of $z_{\mathrm{fg}}$ and overall galaxy properties, and are consistent with our measurements within the uncertainties.

Fig. 6 clearly shows that excess Ly $\alpha$ absorption is detected to transverse distances of at least $D_{\text {tran }} \simeq 3500$ pkpc. A single power law reasonably approximates the dependence of $W_{\lambda}(\operatorname{Ly} \alpha)$ on $D_{\text {tran }}$, with power-law index $\beta=-0.40 \pm 0.01$

$\mathrm{W}_{\lambda}(\mathrm{Ly} \alpha)=(0.429 \pm 0.005 \AA)\left(\frac{D_{\text {tran }}}{\mathrm{pMpc}}\right)^{(-0.40 \pm 0.01)}$.

This power law is also shown in Fig. 6. There is evidence from the new KGPS results for subtle differences in slope over particular ranges of $D_{\text {tran }}$. Specifically, at $D_{\text {tran }}<100 \mathrm{pkpc}, \beta=-1.0 \pm 0.1$; for $100<D_{\text {tran }} / \mathrm{pkpc} \leq 300, \beta=0.0 \pm 0.1$; and $300<D_{\text {tran }} / \mathrm{pkpc} \leq$ 2000, $\beta=-0.48 \pm 0.02$. Details are discussed in Section 4 below.

\subsection{Kinematics}

In order to interpret observations of the kinematics of Ly $\alpha$ absorption, one must first develop a detailed understanding of the effective spectral resolution, including the net contribution of redshift uncertainties. We showed in Section 2.2 that redshift uncertainties are negligible for the KGPS- $z_{\text {neb }}$ galaxies, but that $\simeq 50$ per cent of the KGPS-Full sample whose $z_{\mathrm{fg}}$ was estimated using equations (1)-(3) have larger redshift uncertainties, $\sigma_{\mathrm{z}} \lesssim 140 \mathrm{~km} \mathrm{~s}^{-1}$. In the latter case, redshift uncertainties would make a non-negligible contribution to the effective spectral resolution of the stacked spectra. We determined 
the effective spectral resolution applicable to composites formed from the KGPS- $z_{\text {neb }}$ and KGPS-Full samples separately, using procedures whose details are described in Appendix B.

In fact, Appendix B concludes that the effective spectral resolution, including the contribution of redshift uncertainties, is nearly identical for the two subsamples: $\sigma_{\text {eff }}=189 \mathrm{~km} \mathrm{~s}^{-1}\left(\right.$ KGPS- $\left.z_{\text {neb }}\right)$ and $\sigma_{\text {eff }}=$ $192 \mathrm{~km} \mathrm{~s}^{-1}$ (KGPS-Full). As explained in the appendix, this suggests that the contribution of redshift uncertainties to the effective spectral resolution is small compared to that of the instrumental resolution ${ }^{11}$ : $\sigma_{\mathrm{z} \text {,eff }} \simeq \sqrt{\sigma_{\text {eff }}^{2}(\text { Full })-\sigma_{\text {eff }}^{2}\left(z_{\text {neb }}\right)} \lesssim 60 \mathrm{~km} \mathrm{~s}^{-1}$. This implies that the additional degradation in the effective spectral resolution caused by the use of calibrated $z_{\mathrm{UV}}$ redshifts for the $\sim 50$ percent of the KGPS-Full sample lacking measurements of $z_{\text {neb }}$ is not significant. The contribution is smaller than the redshift error estimated in Section 2.2 for galaxies with only rest-UV measurements; we suggest that the reason for the apparent discrepancy is that the earlier estimate included both the uncertainty in the mean offsets between $z_{\mathrm{UV}}$ and $z_{\text {neb }}$, and the noise associated with the measurement of spectral features in individual spectra, which are effectively averaged out in applying the fits in equations (1)-(4). Nevertheless, we retain the KGPS- $z_{\text {neb }}$ sample as a sanity check to eliminate unknown systematic uncertainties that may be present in the KGPS-Full sample.

As discussed above (Section 3.1), for composite spectra of modest spectral resolution, much of the physical information that could be measured from high-resolution QSO spectra through the same ensemble of sightlines is sacrificed in order to increase the spatial sampling. However, from the smaller samples of QSO sightlines through the CGM of a subset of the KGPS galaxies (Rudie et al. 2012a), we know that the $\operatorname{Ly} \alpha$ absorption profile resolves into a number of individual components of velocity width $\sigma_{v} \simeq 20$ $50 \mathrm{~km} \mathrm{~s}^{-1}$ that do not fully occupy velocity space within $\pm 700 \mathrm{~km} \mathrm{~s}^{-1}$ of the galaxy's systemic redshift. The total absorption strength tends to be dominated by components with $N_{\mathrm{HI}} \gtrsim 10^{14} \mathrm{~cm}^{-2}$, whose Ly $\alpha$ transitions are saturated. Thus, Ly $\alpha$ profiles in stacked spectra at low resolution can be usefully thought of as smoothed, statistical averages of a largely bimodal distribution of pixel intensities that is modulated by whether or not a saturated absorber is present.

Nevertheless, the Ly $\alpha$ line profiles in the KGPS composites encode useful information on the total $\operatorname{Ly} \alpha$ absorption as a function of line-of-sight velocity relative to the galaxy systemic redshifts, and the large number of pairs allows us to map these parameters as a function of $D_{\text {tran }}$. To describe the absorption profiles with sufficient dynamic range, we use the apparent optical depth, defined as

$\tau_{\text {ap }}(v)=-\ln \frac{F(v)}{F_{\text {cont }}(v)}$,

where $F(v)$ is the flux density of the composite spectrum as a function of velocity, $F_{\text {cont }}(v)$ is the continuum level, and $v$ is the line-ofsight velocity relative to $z_{\text {sys }}$. For continuum-normalized spectra, $F_{\text {cont }}(v)=1$. Since we know that H I gas is clumpy and normally saturated, $\tau_{\text {ap }}$ is relatively weakly dependent on $N_{\mathrm{HI}}$ for $N_{\mathrm{HI}}>$ $10^{14} \mathrm{~cm}^{-2}$. Rather, $\tau_{\text {ap }}(v)$ is modulated by both the covering fraction $\left(f_{\mathrm{c}}\right)$ of the clumps, and the typical line-of-sight velocity range over which significant absorption is present.

Fig. 7 shows the map of $\tau_{\text {ap }}$ in the $v_{\text {LOS }}-D_{\text {tran }}$ plane for the KGPS-Full and the KGPS- $z_{\text {neb }}$ sample. Each column in the maps represents a stacked spectrum for sightlines within a bin of $D_{\text {tran }}$, each of which represents an equal logarithmic interval $\Delta\left(\log D_{\text {tran }}\right)=$ 0.3, as in Fig. 6. Assuming that the cumulative number of pairs is

${ }^{11}$ The KGPS-Full and KGPS- $z_{\text {neb }}$ samples comprise a comparable mix of the two LRIS instrumental configurations. proportional to $D_{\text {tran }}^{2}$ (see Fig. 3), the average $D_{\text {tran }}$ weighted by number of sightlines within a bin is $\sim 0.18$ dex greater than the lower bin edge, and $\sim 0.12$ dex smaller than the higher bin edge. The map of $\tau_{\text {ap }}$ versus $D_{\text {tran }}$ is symmetric between the blue and red sides across all $D_{\text {tran }}$ except between $\sim 50$ and $\sim 200 \mathrm{pkpc}$, where the total $\tau_{\text {ap }}$ for $v_{\text {LOS }}>0$ (i.e. the side redshifted with respect to the galaxy systemic redshift) is larger by $>50$ per cent than that of the blueshifted side. The possible origin of the asymmetry is discussed in Section 4.3.

To further increase the SNR, the red and blue sides of the $\tau_{\text {ap }}$ map are folded together in Fig. 8 for the KGPS-Full sample and Fig. 9 for the KGPS- $z_{\text {neb }}$ sample. Also shown in Fig. 8 (top-right) is the map of $\tau_{\text {ap }} \times D_{\text {tran }}^{0.4}$, in order to accentuate the structure at large $D_{\text {tran }}$; the power 0.4 was chosen to approximately counteract the overall decrease in absorption strength with increasing $D_{\text {tran }}$ (equation 12). Equal size bins in $\Delta \log D_{\text {tran }}$ means that fewer sightlines are being averaged when $D_{\text {tran }}$ is smaller, so that the uncertainties are larger for pixels on the left-hand side of the $\tau_{\text {ap }}$ maps: the SNR (per $\AA$ ) maps are shown in the bottom panels of Fig. 8. The SNR was calculated by bootstrap resampling the ensemble of spectra contributing to each $D_{\text {tran }}$ bin 2000 times, and then rescaling so that the units are SNR per $\AA$ in the rest frame of $z_{\mathrm{fg}}$. The values in the uncertainty estimation are also supported by the strength of apparent negative $\tau_{\text {ap }}$ (dotted contours) in the $\tau_{\text {ap }}$ maps, which are consistent with the $1 \sigma$ errors from the bootstrap determinations.

The maps of the KGPS-Full and KGPS- $z_{\text {neb }}$ samples show similar features and are consistent within the uncertainties after convolution of the KGPS- $z_{\text {neb }}$ map with a $\sigma_{z}=60 \mathrm{~km} \mathrm{~s}^{-1}$ Gaussian kernel in the $v_{\text {LOS }}$ direction.

Fig. 8 confirms trends in Ly $\alpha$ absorption strength versus $D_{\text {tran }}$ based on QSO sightlines in KBSS (Rakic et al. 2012; Rudie et al. 2012a; Turner et al. 2014) - a sample with much smaller number of foreground galaxies - and similar surveys at comparable (Adelberger et al. 2003, 2005b; Tummuangpak et al. 2014; Bielby et al. 2017) or lower redshifts (Ryan-Weber 2006; Tejos et al. 2014). It is also consistent with analytical modelling from Kakiichi \& Dijkstra (2018) and the mock observations of QSO sightlines from the Evolution and Assembly of Galaxies and their Environments (EAGLE) simulations (Rakic et al. 2013; Turner et al. 2017), who proposed measuring the typical halo mass of the host galaxies based on matching the observed pixel optical depth as a function of $D_{\text {tran }}$ to dark matter haloes in the simulation.

The KGPS maps show that, at small $D_{\text {tran }}$, excess Ly $\alpha$ absorption reaches velocities $\left|v_{\text {LOS }}\right| \gtrsim 500 \mathrm{~km} \mathrm{~s}^{-1}$ relative to the systemic redshifts of foreground galaxies, and that excess absorption over and above that of the average IGM extends to transverse distances of at least $3.5 \mathrm{pMpc}$ in $D_{\text {tran }}$ direction. Fig. 8 suggests several interesting features in the 2D maps of $\tau_{\text {ap }}$, moving from small to large $D_{\text {tran }}$

(i) A region with high line-of-sight velocity spread $\left(\left\langle\left|v_{\mathrm{LOS}}\right|\right\rangle \gtrsim\right.$ $300-400 \mathrm{~km} \mathrm{~s}^{-1}$ ) on transverse distance scales $D_{\text {tran }} \lesssim 50 \mathrm{pkpc}$.

(ii) An abrupt compression of the $v_{\text {Los }}$ profile beginning near $D_{\text {tran }}$ $\simeq 70 \mathrm{pkpc}$, extending to $D_{\text {tran }} \sim 150 \mathrm{kpc}$, with a local minimum near $D_{\text {tran }} \simeq 100$ pkpc.

(iii) A gradual broadening of the Ly $\alpha$ velocity profile beginning at $D_{\text {tran }} \gtrsim 150 \mathrm{pkpc}$ with $\left|v_{\text {LOS }}\right| \sim 500 \mathrm{~km} \mathrm{~s}^{-1}$ and extending to $>4 \mathrm{pMpc}$ with $\sim 1000 \mathrm{~km} \mathrm{~s}^{-1}$ (most evident in the upper right-hand panel of Fig. 8.)

We show in Section 4.2 that feature (iii) is a natural consequence of the Hubble expansion coupled with decreasing H I overdensity, causing the absorption to become weaker and broader as $D_{\text {tran }}$ increases. Eventually, one would expect that the profile would broaden and weaken until it becomes indistinguishable from the 

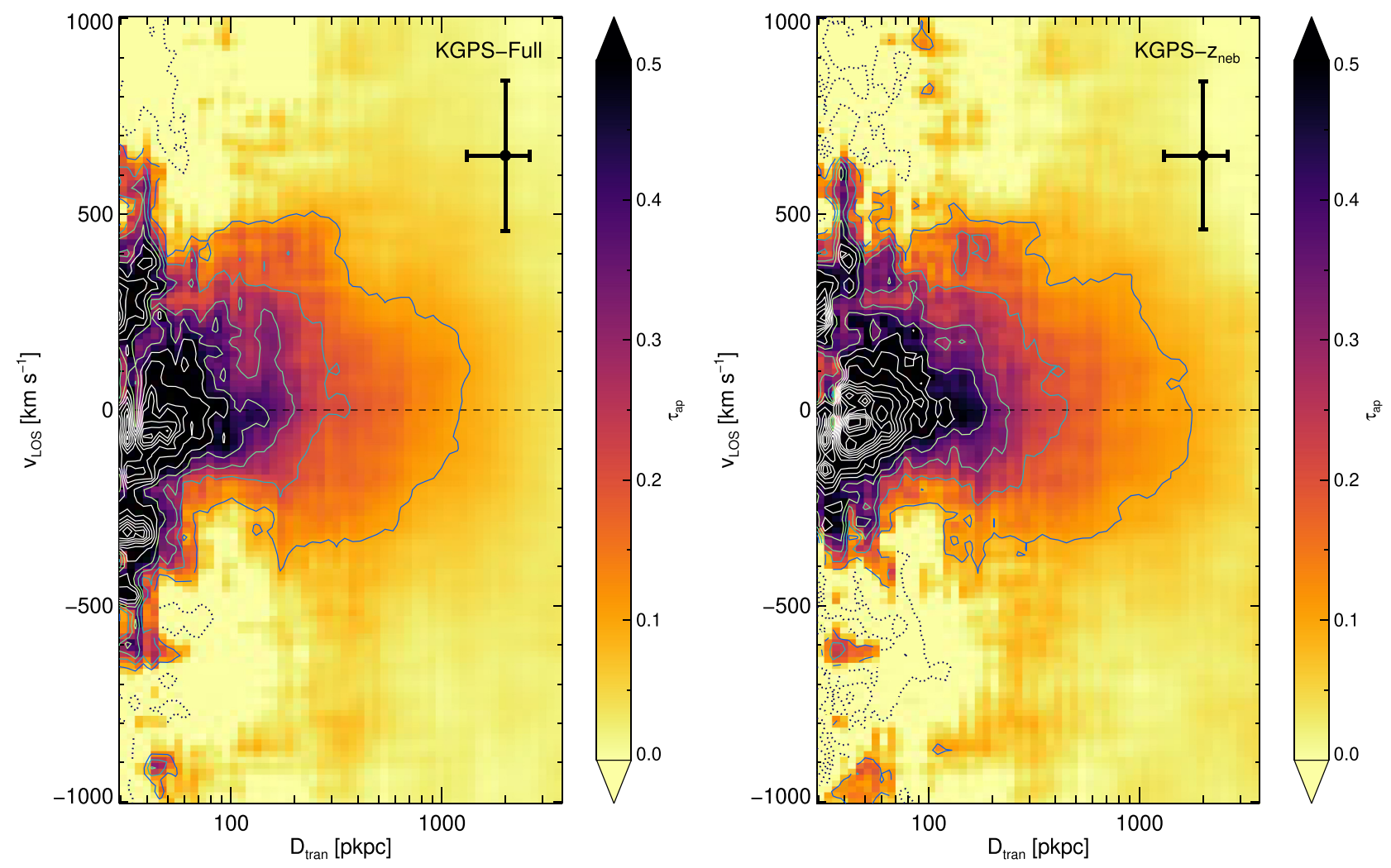

Figure 7. The line-of-sight velocity structure of $\mathrm{HI}$ absorbers around foreground galaxies. Each column of pixels in each plot corresponds to a measurement of $\tau_{\text {ap }}\left(v_{\text {LOS }}\right)$ evaluated from the corresponding composite spectrum in the bin of $D_{\text {tran }}$ made from the KGPS-Full sample (left) and the KGPS- $z_{\text {neb }}$ sample (right). The black dots with horizontal error bars show the range of $D_{\text {tran }}$ used to make each column of the map; the effective velocity resolution of the map is shown as a vertical error bar. Solid contours correspond to positive optical depth $\tau_{\text {ap }}$, with dotted contours indicating negative values, which are consistent with the $1 \sigma$ uncertainties. The contour levels are separated by $\Delta \tau_{\text {ap }}=0.1$.

ambient IGM - recalling that $\tau_{\text {ap }}$ is the excess Ly $\alpha$ optical depth over the intergalactic mean.

We address the likely origin of each of the enumerated features in Section 4.

\section{DISCUSSION}

\subsection{Comparison to cosmological zoom-in simulations}

To aid in the interpretation of the observed $\tau_{\text {ap }}$ maps, we compared them to the distribution of $N_{\mathrm{HI}}$ as a function of $v_{\mathrm{LOS}}$ in a subset of simulations taken from the Feedback In Realistic Environments (FIRE) cosmological zoom-in simulations (Hopkins et al. 2014, 2018). The selected simulations are intended to reproduce LBGlike galaxies at $z \sim 2$; they were originally run at lower spatial resolution using the FIRE-1 feedback model (Faucher-Giguère et al. 2015), but have since been migrated to FIRE-2 with improved mass resolution of $m_{\text {gas }} \sim m_{\text {star }} \sim 700 \mathrm{M}_{\odot}$. We randomly selected a single main simulated galaxy whose halo mass $\left(M_{\mathrm{h}}\right)$, stellar mass $\left(M_{*}\right)$, and star formation rate (SFR) roughly match values inferred for a typical galaxy in the KGPS sample: $\log M_{\mathrm{h}} / \mathrm{M}_{\odot} \sim 12, \log M_{*} / \mathrm{M}_{\odot} \sim 10.5$, and $\left\langle\mathrm{SFR} /\left(\mathrm{M}_{\odot} \mathrm{yr}^{-1}\right)\right\rangle \sim 30$ at $z \sim 2.2$.

We selected 11 snapshots evenly distributed in redshift between $z=2.4$ and $z=2.0 .{ }^{12}$ In each time-step, we chose two random (but

\footnotetext{
${ }^{12}$ The interval $\delta z=0.05$ corresponds to intervals of cosmic time in the range $\delta t \simeq 70 \pm 10 \mathrm{Myr}$ over the redshift range included in our analysis.
}

orthogonal) viewing angles, and calculated the projected quantity $\mathrm{d} N_{\mathrm{HI}} / \mathrm{d} v_{\mathrm{LOS}}$ (a proxy for the observed $\tau_{\text {ap }}$ ) projected on to the 'observed' $D_{\text {tran }}-v_{\text {Los }}$ plane; example maps for the $z=2.20$ time-step are shown in the left-hand panels of Fig. 10. We then median-stacked the $22 N_{\mathrm{HI}}$ maps (11 snapshots for each of two viewing angles); the result is shown in the right-hand panel of Fig. 10). Note that the range shown on the $D_{\text {tran }}$ axis is smaller than that used for the observations (e.g. Fig. 8) because of the limited size of the full-resolution volume of the zoom-in simulation.

Thanks to the large dynamic range in the simulation, one can see that the highest $v_{\text {LOS }}$ values $\left(v_{\text {LOS }} \sim 400-700 \mathrm{~km} \mathrm{~s}^{-1}\right)$ are found within $D_{\text {tran }} \lesssim 50$ pkpc and are associated with material having total $N_{\mathrm{HI}} \simeq \mathrm{d} N_{\mathrm{HI}} / \mathrm{d} v \times \Delta v \simeq 10^{14.5-15.0} \mathrm{~cm}^{-2}$, whereas the slower material with $v_{\mathrm{LOS}}<200 \mathrm{~km} \mathrm{~s}^{-1}$ has $N_{\mathrm{HI}}$ typically $100-1000$ times larger. While essentially all pixels in the map that are significantly above the background in Fig. 10 would give rise to Ly $\alpha$ absorption detectable in KGPS, the low spectral resolution coupled with the inherent loss in dynamic range in $\tau(\operatorname{Ly} \alpha)$ for $\log N_{\mathrm{HI}} \gtrsim 14.5$ makes a detailed quantitative comparison of the KGPS observed map and the simulation map more challenging. Part of the difficulty stems from the limited depth along the line of sight $(\sim 1 \mathrm{pMpc})$ over which the high-resolution zoom-in simulations were conducted, meaning they could be missing potential high-velocity material in the IGM. However, we conclude that the general morphology of the $N_{\mathrm{HI}}$ map from the averaged zoom-in simulations is qualitatively consistent with that of the observed $\tau_{\text {ap }}$ map.

Individual snapshots provide insight into the physical origin of features in the time-averaged map. For example, the most prominent 

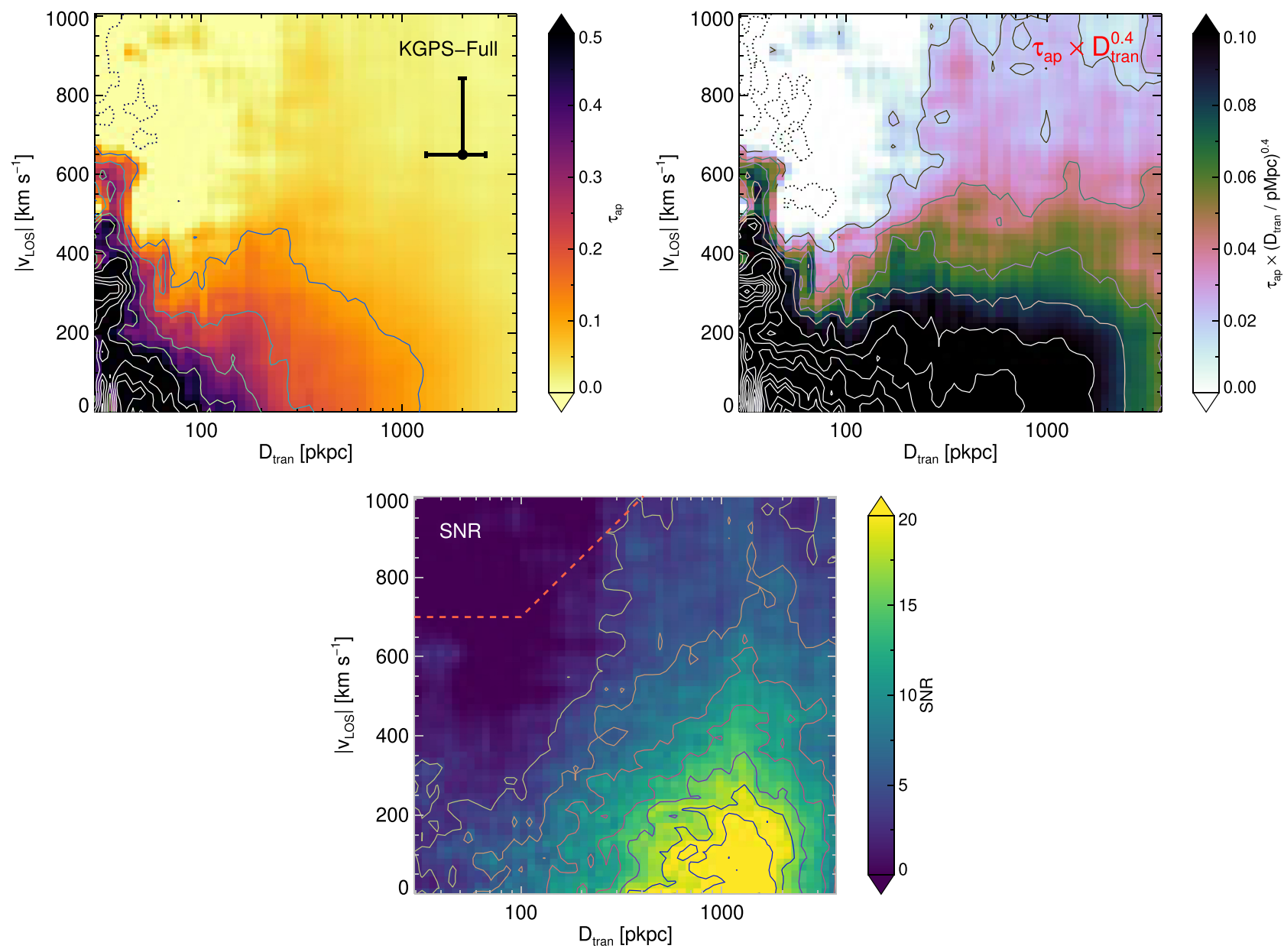

Figure 8. Maps of $\tau_{\text {ap }}$ with the blue and red sides folded to increase the signal-to-noise ratio. The three maps are (left to right, top to bottom): $\tau_{\text {ap }}\left(\left|v_{\text {los }}\right|, D_{\text {tran }}\right)$, $\tau_{\text {ap }} \times D_{\text {tran }}^{0.4}$ (to better illustrate the structure at large $D_{\text {tran }}$ ), and the map of the SNR per $\AA$ of the $\tau_{\text {ap }}$ measurement. The contour decrements are 0.1 for the $\tau_{\text {ap }}$ map, 0.02 for the $\tau_{\text {ap }} \times D_{\text {tran }}^{0.4}$ map, and 3 for the SNR map. The half aperture in which the $W_{\lambda}(\operatorname{Ly} \alpha)$ is measured in Section 3.1 is shown as the orange dashed line in the SNR map.

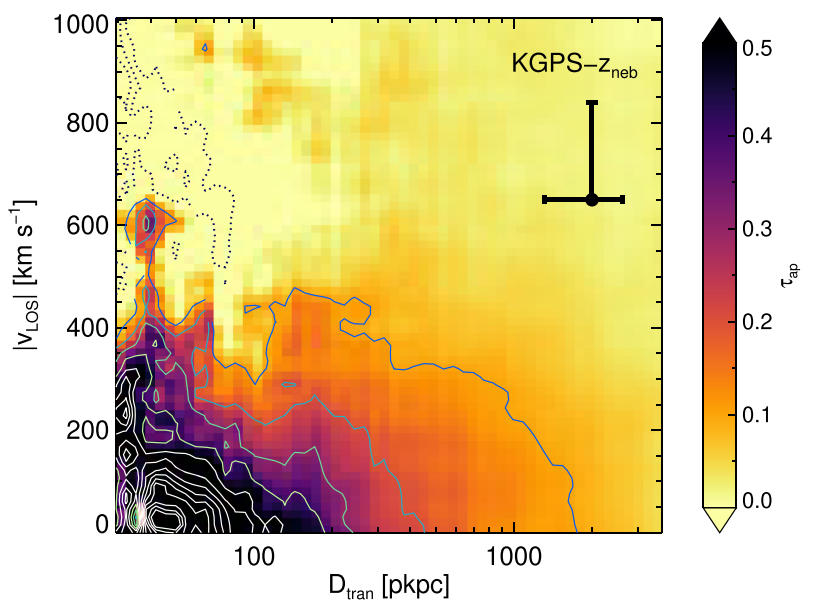

Figure 9. Same as the top-left plot of Fig. 8, but for the KGPS- $z_{\text {neb }}$ sample.

features in Fig. 10 are the vertical 'spikes' evident at $D_{\text {tran }} / \mathrm{pkpc} \gtrsim$ 50 - these are due to the passing of satellite galaxies through the simulation box, giving rise to line-of-sight components of velocity due both to their motion relative to the central galaxy and their internal gas motions, including outflows; e.g. Faucher-Giguère et al. (2016), Anglés-Alcázar et al. (2017), and Hafen et al. (2019). Some of these features remain even in the median of 22 snapshots (righthand panel of Fig. 10), caused by gradual decay of orbits of galaxies destined to merge with the central galaxy. Such features would not be expected to remain in an average over many galaxies, each observed at a particular time (as in the KGPS data), except in so far as there might be a characteristic range of galactocentric radius and relative velocity affected by satellites, so that a net signal might be detected statistically.

With the above caveats in mind, the envelope of $v_{\mathrm{LOS}}$ as a function of $D_{\text {tran }}$ in the simulation resembles that of the KGPS observations in several respects: both the highest velocities and the highest optical depths occur within the central $50 \mathrm{pkpc}$, with a local minimum in the range of $v_{\mathrm{LOS}}$ over which significant $\mathrm{HI}$ is present somewhere in the range 50-100 pkpc, reminiscent of the expected virial radius for a dark matter halo of $\log \left(M_{\mathrm{h}} / \mathrm{M}_{\odot}\right) \sim 12$ of $r_{\text {vir }} \simeq 75-95$ pkpc.

Further insight can be gleaned with reference to Fig. 11, which is essentially a histogram of $\mathrm{HI}$ as a function of galactocentric radius and radial velocity. ${ }^{13}$ Negative (positive) radial velocities indicate

\footnotetext{
${ }^{13}$ The centre of the galaxy is determined by the centre of mass of the dark matter halo.
} 

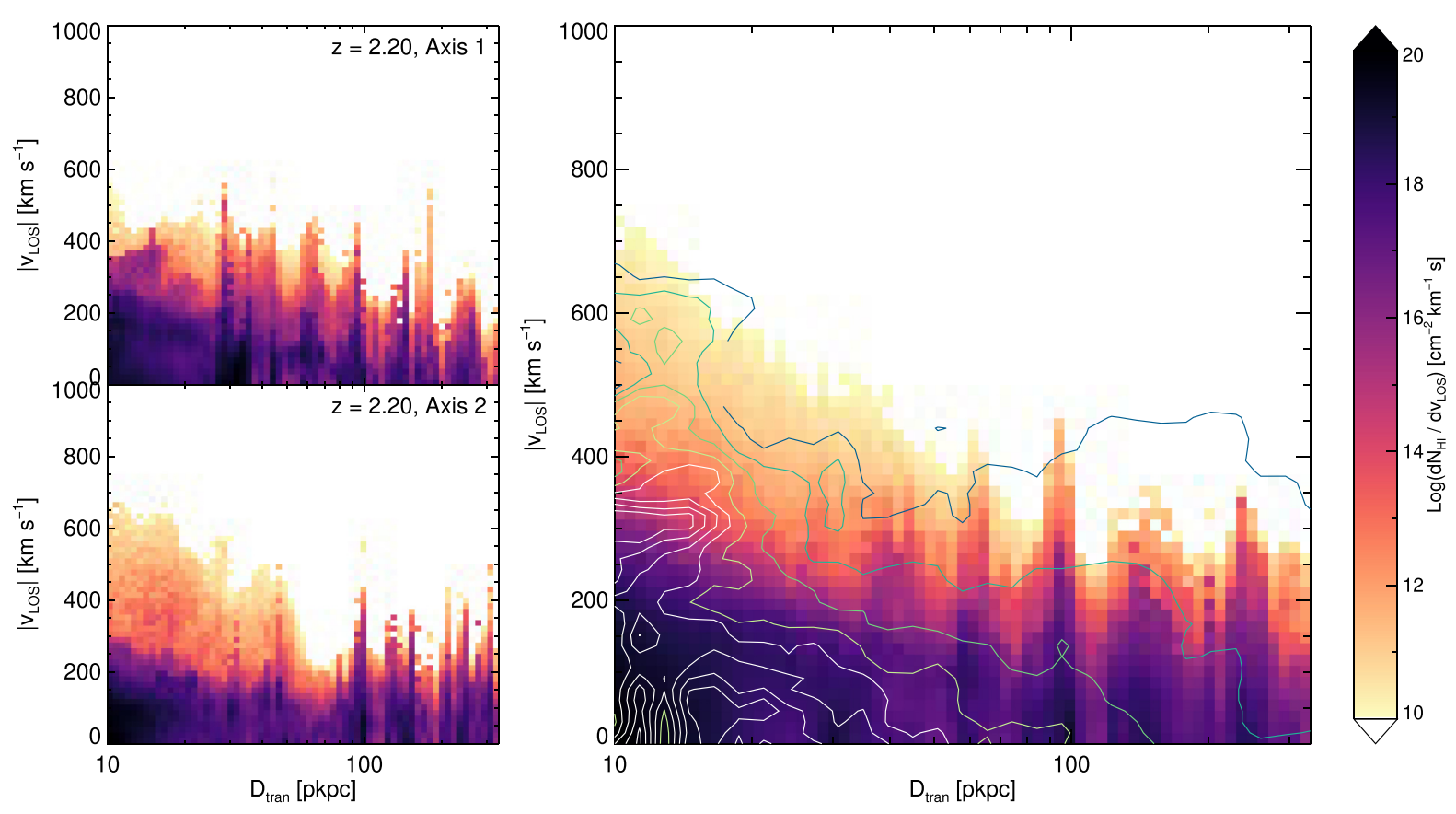

Figure 10. Maps of $\mathrm{d} N_{\mathrm{HI}} / \mathrm{d} v_{\mathrm{LOS}}$ in the FIRE-2 simulation (h350), as seen by an observer, projected on to the $v_{\mathrm{LOS}}-D_{\text {tran }}$ plane. Left: Snapshots from a single time-step $(z=2.20)$ as viewed from two orthogonal viewing angles. Right: The median-stack of 22 such maps: 11 time-steps at intervals of $\delta z=0.05$ for $2.0 \leq$ $z \leq 2.4$ from each of two orthogonal viewing angles. Contours based on the observed map of $\tau_{\text {ap }}$ for the KGPS-Full sample (Fig. 8) are overlaid for comparison. Note that the $D_{\text {tran }}$-axis has been zoomed in from that of Fig. 8 because of the limited size (a few times $r_{\text {vir }}$ ) of the high-resolution zoom-in region of the FIRE-2 simulation.

motion towards (away from) the centre of the galaxy. This type of diagram makes it easier to distinguish gas with substantial outflow velocities, which cause the clear asymmetry of the radial velocity distribution relative to the galaxy centre of mass rest frame. In this particular case, there is neutral hydrogen with $v_{\mathrm{r}}>300 \mathrm{~km} \mathrm{~s}^{-1}$ at galactocentric radii from $r=0$ to $r \simeq 200 \mathrm{pkpc}$, but essentially no gas with $v_{\mathrm{r}}<-300 \mathrm{~km} \mathrm{~s}^{-1}$ except for that due to satellite galaxies. If one looks at the same diagram in successive time-steps, it is clear that the high velocities are associated with episodes of high SFR, and that this particular galaxy is experiencing such an episode at the present time-step, which produces most of the high velocity gas with $r \lesssim 50 \mathrm{kpc}$; the 'plume' of high velocity material that peaks near $r=100 \mathrm{pkpc}$ is a remnant of a similar episode that occurred $\sim 100$ Myr earlier that has propagated to larger galactocentric radii with somewhat reduced $v_{\mathrm{r}}$. Such episodic star formation and outflows are typical in high- $z$ galaxies in the FIRE simulation (Muratov et al. 2015). Such plumes are also seen in IllustrisTNG simulations (Nelson et al. 2019), although they are generally due to the implementation of AGN feedback in higher mass haloes, and thus have even larger velocities. One can also see an accretion stream that has $v_{\mathrm{r}} \simeq 0$ at $r \simeq 200 \mathrm{kpc}$, and evidently has accelerated to $v_{\mathrm{r}} \simeq$ $-200 \mathrm{~km} \mathrm{~s}^{-1}$ by $r \sim 50 \mathrm{pkpc}$. However, the bulk of the H I mass within the virial radius is not obviously accreting or outflowing, with $\left|v_{\mathrm{r}}\right| \lesssim 250 \mathrm{~km} \mathrm{~s}^{-1}$.

A halo with $M_{\mathrm{h}}=10^{12} \mathrm{M}_{\odot}$ and $r_{\mathrm{vir}} \simeq 90 \mathrm{kpc}$ has a circular velocity of $\simeq 220 \mathrm{~km} \mathrm{~s}^{-1}$, so that if gas were on random orbits one would expect to measure a $1 \mathrm{D}$ velocity dispersion (more or less independent of radius, for realistic mass profiles) of $\sigma_{1 \mathrm{D}} \simeq 220 / \sqrt{3} \simeq 130 \mathrm{~km} \mathrm{~s}^{-1}$, with $\sim 95$ per cent of particle velocities expected to lie within $\left|v_{\text {LOS }}\right|$ $\lesssim 260 \mathrm{~km} \mathrm{~s}^{-1}$. These expectations could be modulated by the prevalence of mostly circular or mostly radial orbits, of course. However, in a statistical sense, gravitationally induced motions could contribute a fraction of the most extreme velocities (i.e. $\left|v_{\mathrm{r}}\right|>$ $300 \mathrm{~km} \mathrm{~s}^{-1}$ within $\lesssim 2 R_{\mathrm{vir}}$ ), but are not enough to make up the whole.

\subsection{A simple analytical model}

\subsubsection{Context}

Inspired by comparisons to the cosmological zoom-in simulations, and to offer an explanation for the general shape of the $\tau_{\text {ap }}$ map before discussing the details, we constructed a two-component analytical model intended to capture the salient features of Fig. 8 .

To construct a model in 3D physical space, a parameter must be chosen to represent Ly $\alpha$ absorption strength. As discussed in Section $2.4, \tau_{\text {ap }}\left(v_{\mathrm{LOS}}\right)$ is affected by a combination of the total $N_{\mathrm{HI}}$, which is known to depend on $D_{\text {tran }}$ (e.g. Rudie et al. 2012a), the average distribution of $v_{\text {LOS }}$ at a given $D_{\text {tran }}$, and the fraction of sightlines that give rise to detectable absorption at a given $D_{\text {tran }}$. The relative importance of these effects depends on galactocentric distance (i.e. $D_{\text {tran }}$ ) in a complex manner that cannot be resolved from an ensemble of low-resolution spectra, which also cannot be expected to reveal the level of detail that could be measured from individual sightlines observed at very high spectral resolution. However, some general statements about the 'sub-grid' behaviour of Ly $\alpha$ absorption may be helpful in providing some intuition.

At large $D_{\text {tran }}$, given the minimum total equivalent width detected of $\simeq 0.2 \AA$ (see Fig. 6), the lowest column density that could be measured for a single Ly $\alpha$ absorption line is $\log N_{\mathrm{HI}} \simeq 13.6$, assuming a linear curve of growth; for a typical value of the Doppler parameter $b_{\mathrm{d}} \simeq 25 \mathrm{~km} \mathrm{~s}^{-1}\left(\sigma_{\mathrm{d}} \equiv b_{\mathrm{d}} / \sqrt{2} \simeq 17.7 \mathrm{~km} \mathrm{~s}^{-1}\right)$, the single line would have an optical depth at line centre of $\tau_{0} \simeq 2.4$, which if resolved would produce a minimum flux density relative to the 


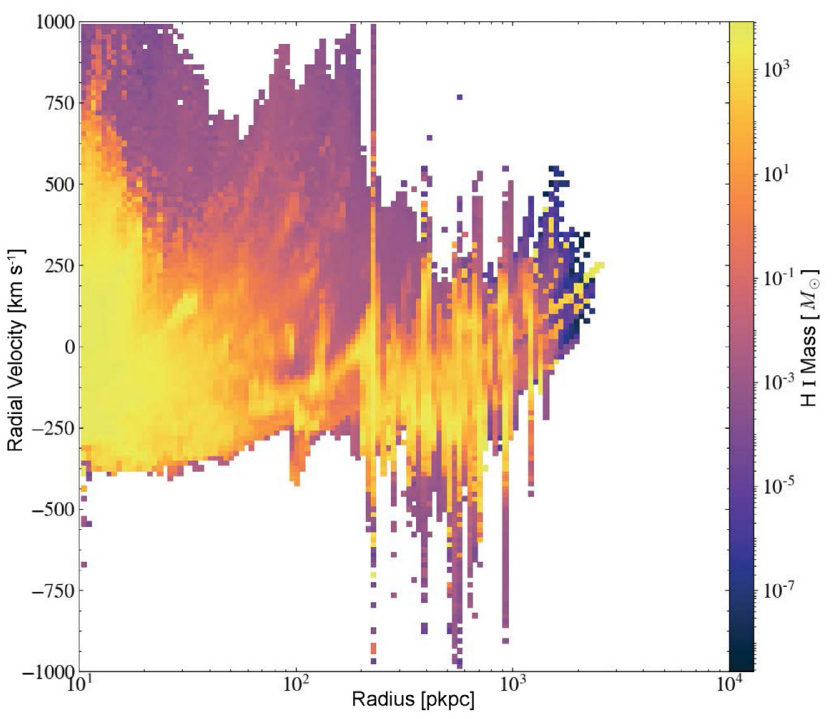

Figure 11. The galactocentric radial velocity versus galactocentric radius for neutral hydrogen in the same $z=2.20$ snapshot as shown in the left-hand panels of Fig. 10. Positive and negative values indicate net outward and net inward radial motion, respectively. The vertical strips are due to the outflow of the satellite galaxies. The colourbar represents the total $\mathrm{H}$ I mass in each pixel. The gradual decrease of $\mathrm{HI}$ content beyond $500 \mathrm{pkpc}$ is artificial because of the limited volume within which the gas simulation is conducted.

continuum of $F_{v, 0} \sim 0.09$. However, given the effective spectral resolution of $\sigma_{\text {eff }} \simeq 190 \mathrm{~km} \mathrm{~s}^{-1}$, the observed line profile of the same line would have $\sigma_{\mathrm{LOS}} \simeq 190 \mathrm{~km} \mathrm{~s}^{-1}$ and central apparent optical depth of only $\tau_{\text {ap, } 0} \simeq 0.2$, or $F_{v, 0} / F_{v \text {, cont }} \simeq 0.82$. In fact, at large $D_{\text {tran }}$ we measure $\sigma_{\mathrm{LOS}} \simeq 600 \mathrm{~km} \mathrm{~s}^{-1}$, and a maximum apparent optical depth $\tau_{\text {ap }} \lesssim 0.06$. This suggests that at large $D_{\text {tran }}$ we are measuring a small total $N_{\mathrm{HI}}$ excess $\left[\log \left(N_{\mathrm{HI}} / \mathrm{cm}^{-2}\right)<14\right]$ spread over a large range of $v_{\text {LOS }}$; the apparent line width is best interpreted as a probability distribution in $v_{\mathrm{LOS}}$ of the small excess absorption over that of the general IGM.

At small $D_{\text {tran }}$, the situation is very different; in most cases, if observed at high spectral resolution, one would see several components within a few hundred $\mathrm{km} \mathrm{s}^{-1}$ of the galaxy systemic velocity, most of which would be strongly saturated $\left(\log N_{\mathrm{H}} / \mathrm{cm}^{-2}\right.$ $\gtrsim 14.5)$ and complexes of absorption could often produce large swaths of velocity space with $F_{v}=0$ (e.g. Rudie et al. 2012a). At $D_{\text {tran }} \lesssim 50 \mathrm{kpc}$, the total $W_{\lambda}(\operatorname{Ly} \alpha) \simeq 2 \AA$ with maximum $\tau_{\text {ap }, 0} \simeq 1$ and $\sigma_{\mathrm{LOS}} \sim 320 \mathrm{~km} \mathrm{~s}^{-1}$. Once saturation occurs, the equivalent width contributed by individual absorbers grows very slowly with increasing $N_{\mathrm{HI}}$ until Lorentzian damping wings begin to become important $\left(\log \left(N_{\mathrm{HI}} / \mathrm{cm}^{-2}\right) \gtrsim 19.0\right)$. At high spectral resolution, $\exp \left(-\tau_{0}\right) \simeq 0$ independent of $N_{\mathrm{HI}}$ so that additional absorbers in the same range of $v_{\text {LOS }}$ might have little or no effect on the absorption profile, especially when observed at low spectral resolution. However, a relatively small amount of H I with larger $\left|v_{\text {LOS }}\right|$ - if it is a common feature of $D_{\text {tran }}$ $\lesssim 50$ pkpc sightlines - would be easily measured.

\subsubsection{Parametrization}

Fig. 12 illustrates the parametrization of our model. In the interest of simplicity, our model makes no attempt to capture the detailed radiative processes (e.g. Kakiichi \& Dijkstra 2018) of individual absorption components that would be revealed by high-resolution QSO spectra. Instead, in order to unify the two extreme scenarios

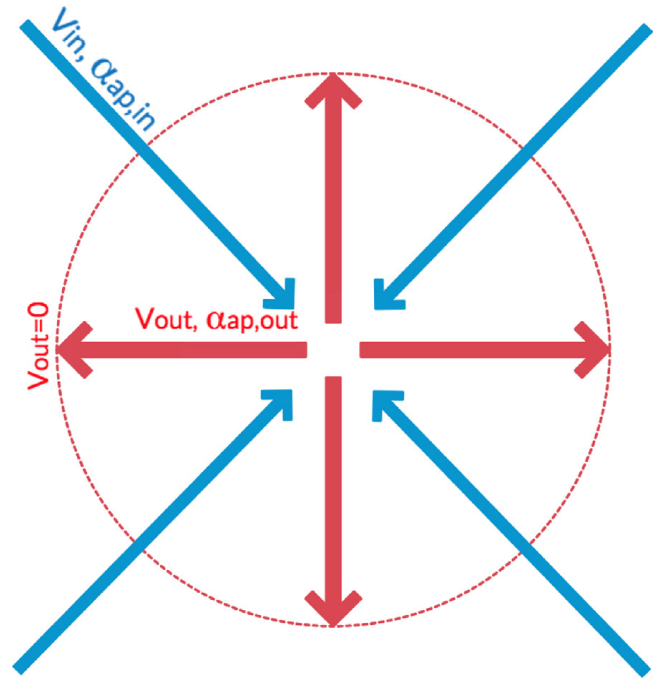

Figure 12. A cartoon illustration of the parametrization of the analytical model described in Section 4.2.2. The model comprises two isotropic, noninteracting, purely radial components: 'outflow' (red) and 'inflow' (blue). Each component has free parameters describing its radial velocity profile $v(r)$ and apparent absorption coefficient $\alpha_{\text {ap }}(r)$ (defined in equations 14-16). The outflow component is truncated at the point that it slows to $v_{\text {out }}=0$.

described in Section 4.2.1 above, we treated HI in the CGM as a continuous medium in which the absorption strength per unit pathlength is represented by an absorption coefficient

$\alpha_{\mathrm{ap}}=\frac{\mathrm{d} \tau_{\mathrm{ap}}}{\mathrm{d} l}$,

where $\mathrm{d} l$ is the differential path-length. In essence, $\alpha_{\text {ap }}$ represents the overdensity of $\mathrm{H}$ I relative to the average in the IGM, accounting for some of the non-linearity that results from curve-of-growth effects that remain unresolved by the data.

We assumed that the HI surrounding galaxies are composed of two isotropic (non-interacting) components: one moves radially outward ('outflow'), the other radially inward ('inflow'). Each has $\alpha_{\text {ap }}$ parametrized as an independent radial power law

$\alpha_{\text {ap, out }}(r)=\alpha_{0, \text { out }} r_{100}^{-\gamma_{\text {out }}}$

$\alpha_{\text {ap,in }}(r)=\alpha_{0, \text { in }} r_{100}^{-\gamma_{\text {in }}}$,

where $\alpha_{0, \text { out }}$ and $\alpha_{0 \text {, in }}$ are normalization constants, $\gamma_{\text {out }}$ and $\gamma_{\text {in }}$ are power-law indices, and $r_{100}=r / 100 \mathrm{pkpc}$ is the galactocentric radius.

For simplicity, we assumed that the velocity fields of the two components are also isotropic and purely radial; clearly this is unrealistic. However, since only the line-of-sight component of gas velocity is measured, random motions of gas moving in the galaxy potential are partly degenerate with our treatment of gas accretion. In the context of the simplified model, one can think of the 'inflow' component as a proxy for all gas motions that are induced by the galaxy's potential. Under these assumptions, there is a simple geometric relationship between the line-of-sight component of velocity $v_{\text {LOS }}\left(D_{\text {tran }}, l\right)$ at each point along a sightline through the CGM and the radial velocity $v_{\mathrm{r}}(r)$

$v_{\mathrm{LOS}}\left(D_{\mathrm{tran}}, l\right)=\frac{l}{r} v_{\mathrm{r}}(r)$,

where $l$ is the line-of-sight coordinate distance measured from the tangent point where $v_{\text {LOS }}=0$, i.e. where $r=D_{\text {tran }}$, and in general, $r^{2}=l^{2}+D_{\text {tran }}^{2}$. Within this paradigm, specification of $v_{\text {out }}(r), v_{\text {in }}(r)$, 
$\alpha_{\text {ap, out }}(r)$, and $\alpha_{\text {ap, in }}(r)$ can be transformed to maps of $\tau_{\text {ap }}\left(D_{\text {tran }}, v_{\text {LOS }}\right)$ directly analogous to those in Fig. 8.

For the outflow component velocity field $v_{\text {out }}(r)$, we assume that the gas has been accelerated to an initial 'launch' velocity $v_{1}$ at a galactocentric radius $r=1 \mathrm{pkpc}$, beyond which its trajectory is assumed to be purely ballistic (i.e. no pressure gradient or mass loading is accounted for) within an NFW halo (Navarro, Frenk \& White 1996) density profile. The outflow component is truncated (i.e. $\left.\alpha_{\text {ap, out }}=0\right)$ at the radius where $v_{\text {out }}(r) \rightarrow 0$. With these assumptions

$v_{\text {out }}(r)=\sqrt{v_{1}^{2}+A\left(-\ln \frac{R_{\mathrm{S}}+1}{R_{\mathrm{s}}}+\frac{1}{r} \ln \frac{R_{\mathrm{s}}+r}{R_{\mathrm{S}}}\right)}$,

where $R_{\mathrm{S}}$ is the NFW scale radius in pkpc, and

$$
\begin{aligned}
A & =\frac{8 \pi G \rho_{0} R_{\mathrm{s}}^{3}}{1 \mathrm{pkpc}} \\
& \simeq 1.2 \times 10^{7} \mathrm{~km}^{2} \mathrm{~s}^{-2} .
\end{aligned}
$$

Following Klypin et al. (2016), for a $M_{\mathrm{h}}=10^{12} \mathrm{M}_{\odot}$ NFW halo with concentration parameter $c=3.3$ at $z=2.3, R_{s}=27$ pkpc (i.e. $R_{\text {vir }}$ $\simeq 90 \mathrm{pkpc}$ ).

For the inflow component, we make the simplifying assumption that $v_{\text {in }}(r)$ is just a constant velocity offset relative to the Hubble expansion (similar to Kaiser 1987)

$v_{\text {in }}(r)=v_{\text {offset }}+H(z) r$,

where $H(z)$ is the Hubble parameter at redshift $z$. For our assumed $\Lambda \mathrm{CDM}$ cosmology and given the median redshift of the KGPS foreground galaxies, $\langle z\rangle=2.2$, we set $H(z)=227 \mathrm{~km} \mathrm{~s}^{-1} \mathrm{pMpc}^{-1}$.

Given the parametrization in equations (15), (16), (18), and (21), for each realization of the MCMC we projected both components independently to the $\left|v_{\mathrm{LOS}}\right|-D_{\text {tran }}$ plane, in the process of which $\alpha_{\text {ap }}$ was converted to $\tau_{\text {ap }}$ by integration. Subsequently, $\tau_{\text {ap, in }}$ and $\tau_{\text {ap, out }}$ are added in $D_{\text {tran }}-v_{\text {LOS }}$ space, and convolved with the effective resolution of the observed $\tau_{\text {ap }}$ maps as determined in Section 3.2.

\subsubsection{Results}

The best-fitting model parameters were estimated using a Markov chain Monte Carlo (MCMC) method to fit to the observed maps of $\tau_{\text {ap }}$. Prior to fitting, in order to reduce pixel-to-pixel correlations, the observed maps were resampled to a grid with $\Delta v_{\mathrm{LOS}}=101 \mathrm{~km} \mathrm{~s}^{-1}$ and $\Delta \log \left(D_{\text {tran }}\right)=0.126$. These pixel dimensions represent one standard deviation of the fitted 2D Gaussian covariance profile determined from bootstrap resampling of oversampled maps.

Even with the simplified model, we found it was necessary to fit the inflow and outflow serially, rather than simultaneously, to achieve convergence in the MCMC. Specifically, the inflow component was fit in the region of $D_{\text {tran }}>400 \mathrm{pkpc}$, assuming that this part of the $\tau_{\text {ap }}$ map is dominated by inflow. ${ }^{14}$ Once the inflow parameters $\left(\alpha_{0, \text { in }}, \gamma_{\text {in }}\right.$, $v_{\text {offset }}$ ) were obtained, they were held fixed and combined with the as-yet-undetermined outflow model to fit the whole $\tau_{\text {ap }}$ map. For the inflow MCMC, the priors were assumed to be flat in linear space with positive values (except for $v_{\text {offset }}$, which is negative for inflow). The outflow MCMC, however, adopts the most probable posterior of the inflow parameters and fixes them; this may be viewed as a strong prior on the resulting outflow parameters, which are otherwise assumed to be flat and positive valued.

\footnotetext{
${ }^{14}$ The region was chosen by eye based on Fig. 8 to minimize the contamination from outflow.
}

Table 3. Best-fitting model parameters.

\begin{tabular}{lccc}
\hline & Parameter & KGPS-Full & KGPS- $z_{\text {neb }}$ \\
\hline Inflow & $v_{\text {offset }}\left(\mathrm{km} \mathrm{s}^{-1}\right)$ & $-84_{-6}^{+6}$ & $-110_{-9}^{+8}$ \\
& $\alpha_{0, \text { in }}\left(\mathrm{pkpc}^{-1}\right)$ & $0.0083_{-0.0008}^{+0.0011}$ & $0.0095_{-0.0013}^{+0.0018}$ \\
& $\gamma_{\text {in }}$ & $0.58_{-0.02}^{+0.03}$ & $0.62_{-0.03}^{+0.04}$ \\
Outflow & $v_{1}\left(\mathrm{~km} \mathrm{~s}^{-1}\right)$ & $603_{-11}^{+5}$ & $575_{-3}^{+7}$ \\
& $\alpha_{0, \text { out }}\left(\mathrm{pkpc}^{-1}\right)$ & $0.031_{-0.008}^{+0.010}$ & $0.034_{-0.012}^{+0.057}$ \\
& $\gamma_{\text {out }}$ & $2.0_{-0.1}^{+0.1}$ & $1.1_{-0.1}^{+0.4}$ \\
\hline
\end{tabular}

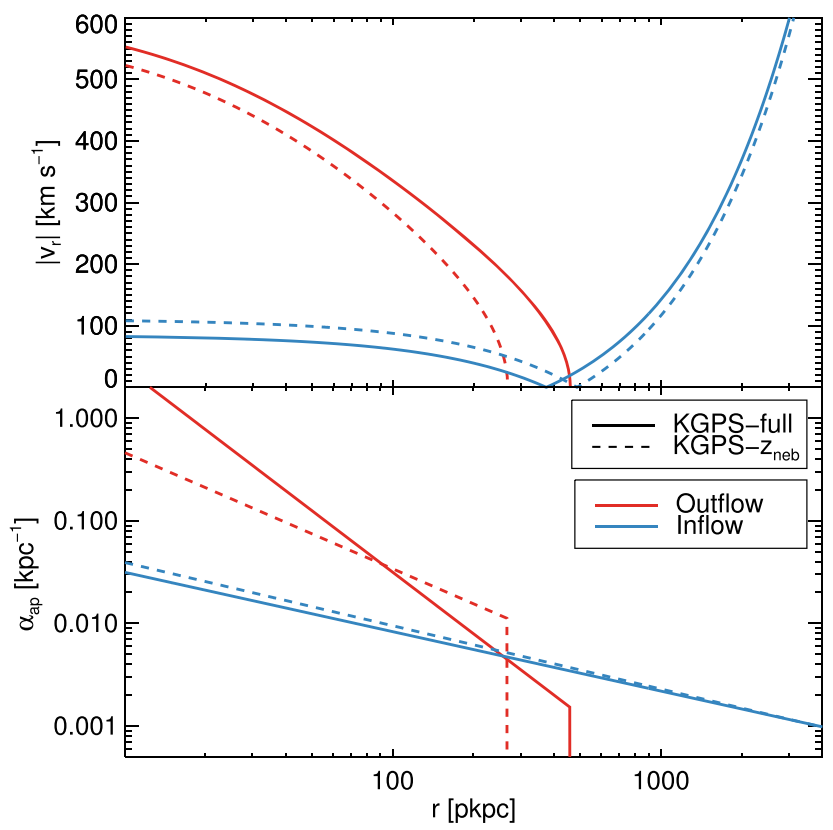

Figure 13. The best-fitting radial profiles of $v_{\mathrm{r}}$ and $\alpha_{\mathrm{ap}}$ as functions of galactocentric radius (i.e. before projection to the observed $v_{\mathrm{LOS}}-D_{\text {tran }}$ plane). The model parameters are as in Table 3. The red curves correspond to the outflow component and blue curves to the inflow. The best-fitting model for the KGPS-Full sample is shown with solid lines, while the best-fitting model for the KGPS- $z_{\text {neb }}$ is shown with dashed lines.

The best-fitting model parameters are summarized in Table 3, and the $\alpha(r)$ and $v_{r}(r)$ profiles for the KGPS-Full and the KGPS- $z_{\text {neb }}$ samples are shown in Fig. 13. In general, the fitted parameters for the two maps are consistent with one another within the estimated uncertainties; the main difference is in the exponent $\gamma_{\text {out }}$, the inferred radial dependence of the outflow component (see Fig. 13). In the case of the sparser KGPS- $z_{\text {neb }}$ sample, $\gamma_{\text {out }}$ is not very well constrained, likely due to sample variance at small $D_{\text {tran }}$ caused by the relatively small number of independent sightlines. Fig. 14 compares the observed and modelled $\tau_{\text {ap }}$ maps and the residual map for the KGPSFull sample. The best-fitting model reproduces the general features of Fig. 8 reasonably well.

The best-fitting outflow models above are, on the face of it, inconsistent with the kinematic outflow model of S2010, in which the radial velocity was constrained primarily by 'down-the-barrel' (DTB) profiles of blue-shifted low-ionizaton metal lines (rather than $\mathrm{HI}$ ) in galaxy spectra. In the S2010 models, most of the acceleration of outflowing material was inferred to occur within $r \lesssim 5-10 \mathrm{pkpc}$ of the galaxy centre, with few constraints on $v(r)$ at larger radii; however, we note that the asymptotic velocity of the fastest-moving 

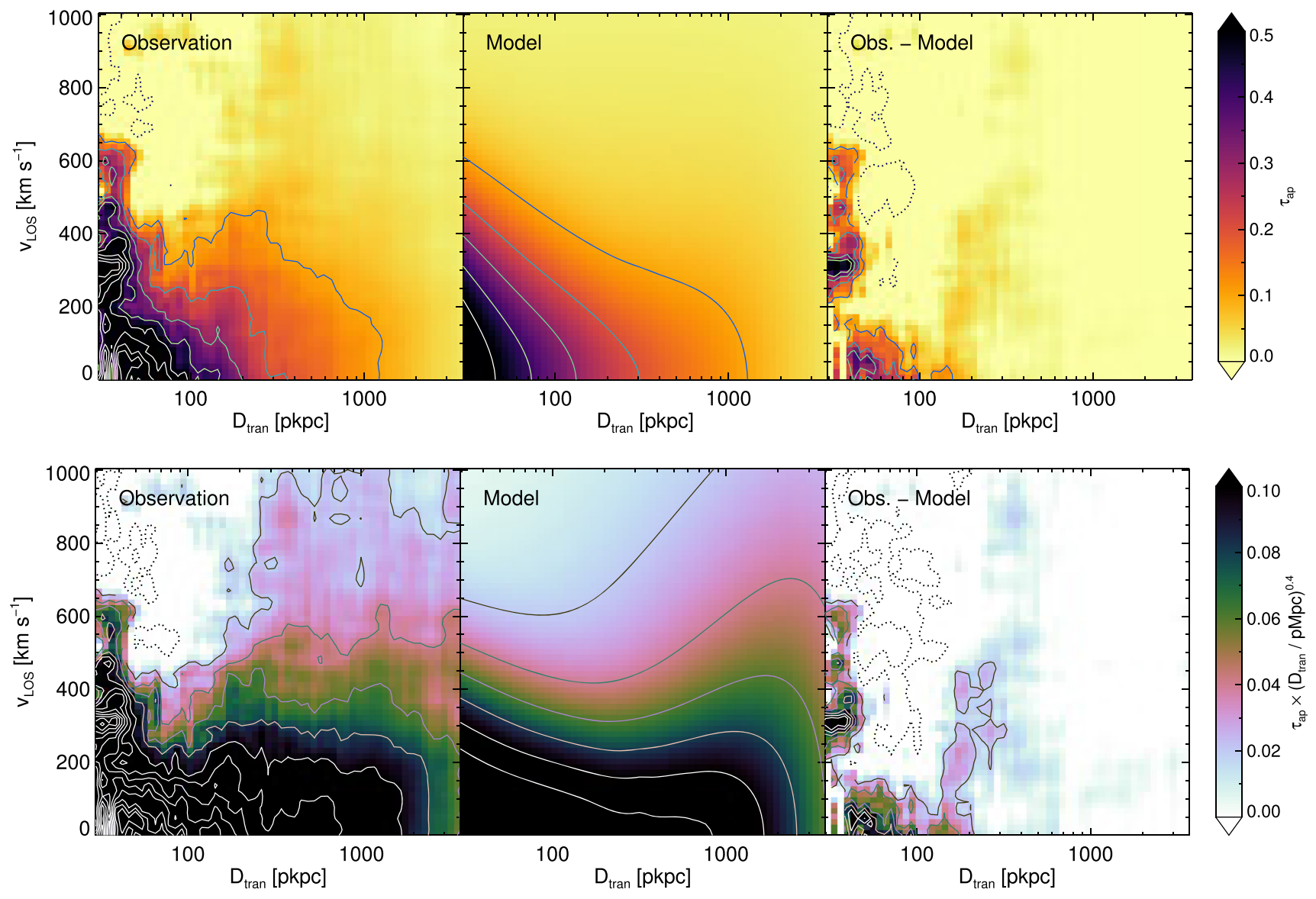

Figure 14. Comparison of the observed KGPS-Full map (left) with the best-fitting model (middle). The right-most plots are residuals after subtraction of the model from the observed map. The top panel shows $\tau_{\text {ap }}$ as a function of $D_{\text {tran }}$ and $v_{\text {Los }}$, while the bottom panel shows $\tau_{\text {ap }}$ multiplied by $D_{\text {tran }}^{0.4}$. The colourbars and contour levels are identical to those shown in Fig. 8.

material in the S2010 models was $\sim 600-700 \mathrm{~km} \mathrm{~s}^{-1}$, similar to the highest detected $v_{\text {LOS }}$ at small $D_{\text {tran }}$ in the KGPS optical depth maps (e.g. Fig. 8). S2010 did not use information from measurements of $v_{\text {LOS }}$ profiles as a function of $D_{\text {tran }}$, which has provided the most important new constraints in this work.

Additionally, although various hydrodynamical simulations of galaxy formation differ substantially in their spatial resolution and feedback implementation, our best-fitting outflow velocity of $\sim 600 \mathrm{~km} \mathrm{~s}^{-1}$ is consistent with the upper range of gas velocities found for simulated galaxies with similar halo mass and redshift. For example, in the FIRE zoom-in simulations, the 95th percentile gasphase velocity is $\sim 600 \mathrm{~km} \mathrm{~s}^{-1}$ at $0.25 R_{\text {vir }}(r \simeq 25 \mathrm{pkpc}$ ) (Muratov et al. 2015); for larger volume simulations, which depend to a greater extent on 'sub-grid' treatment of feedback physics, the level of agreement depends on the simulation suite: in the IllustrisTNG simulations, the 95th percentile gas-phase velocity is $\sim 650 \mathrm{~km} \mathrm{~s}^{-1}$ at $r=10$ pkpc (Nelson et al. 2019), whereas in the EAGLE simulations, the 90 percentile gas velocity between 0.1 and $0.2 r_{\text {vir }}$ (equivalent to $r \sim 10-20 \mathrm{pkpc}$ for the galaxies in our observed sample) is smaller, $\sim 350 \mathrm{~km} \mathrm{~s}^{-1}$ (Mitchell et al. 2020). It is important to note that the comparison of our observations with the FIRE simulation in Section 4.1 is more direct, since it was confined to neutral $\mathrm{H}$ in the simulation box, while the results quoted above pertain to all outflowing gas, regardless of physical state. It will be important in future to examine simulation results in terms of parameters that are most directly comparable to the available observational constraints.

The simple model framework we adopted is not intended to reproduce all aspects of what is undoubtedly a more complex situation in reality. There are clearly features of the observations that are not successfully captured by the model; these are discussed in Section 4.2.4 below. Here, we examine whether its basic assumptions - that both infall and outflows are required to reproduce the general behaviour of the observed $\tau_{\text {ap }}$ maps - are justified.

One questionable assumption we adopted for the fiducial model is the kinematic nature of the outflow, which was launched from $r=1 \mathrm{pkpc}$ at high velocity and subsequently affected only by gravity. It neglects the possible effects of a pressure gradient in the halo, through which buoyancy forces could conceivably counter-act gravity in determining the velocity of outflowing material as it moves to larger radii (e.g. Ji et al. 2020). It also assumes that no ambient gas is entrained by the outflow (i.e. no additional mass loading) as it moves outward, which could reduce the outflow velocity $v_{\text {out }}(r)$ more rapidly than the model presented above.

As a test, we relaxed the ballistic assumption and considered a model in which the outflow velocity is simply a power-law function of galactocentric radius, $v_{\text {out }}(r)=v_{1} r^{\beta}$. With this parametrization, we found a best-fitting $\beta=-0.7_{-0.2}^{+0.5}$, while the $\alpha_{\text {out }}(r)$ remains largely unchanged for the KGPS-Full sample (see Fig. 15). The alternative model does result in a more rapid decline in the range of $v_{\mathrm{LOS}}$ at $D_{\text {tran }}$ 

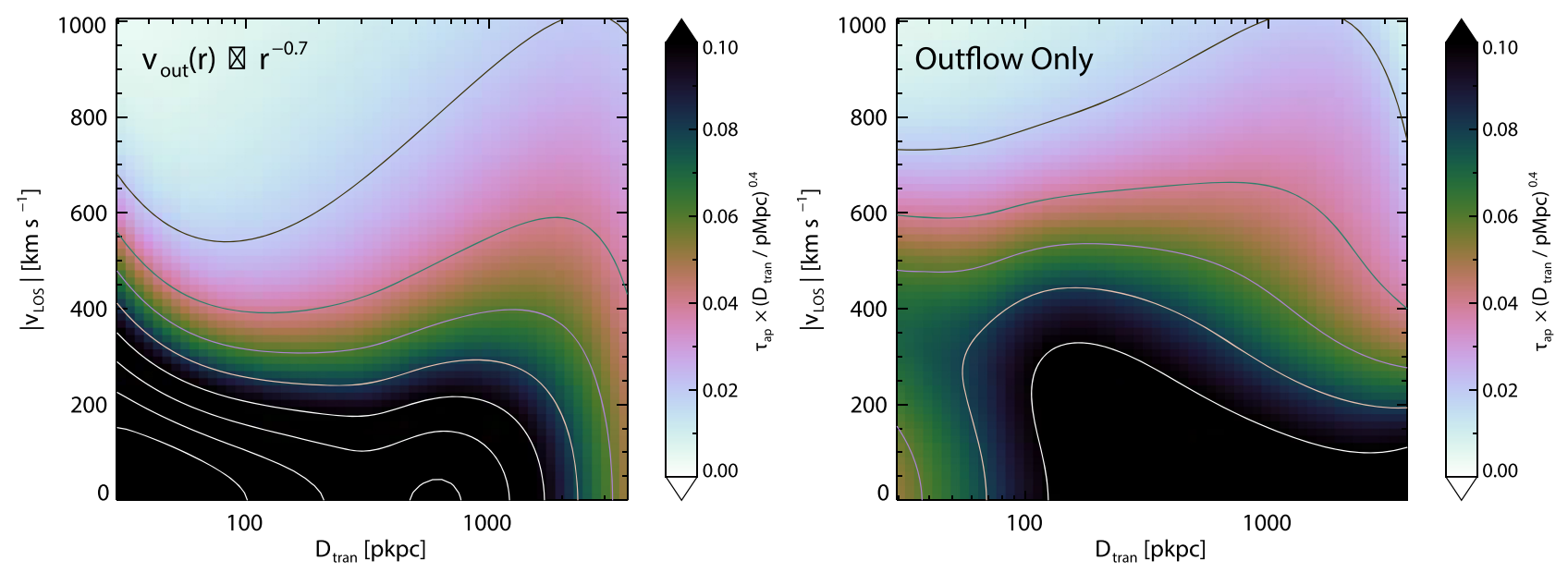

Figure 15. The best-fitting parametrizations of alternative models, to be compared with that of the fiducial model shown in Fig. 14. Both maps show the quantity $\tau_{\text {ap }} \times D_{\text {tarn }}^{-0.4}$, as in the bottom panels of Fig. 14. (Left:) Same as fiducial, but with the outflow velocity $v_{\text {out }}(r)$ as power law. (Right:) A (ballistic) outflow-only model that eliminates the infall component completely. The power-law model fits the data similarly to the fiducial; the outflow-only model is a poor fit.

$\sim 100 \mathrm{pkpc}$ (and thus slightly closer to the observed map in the same range) but the overall fit has $\chi^{2}$ similar to that of our fiducial model. Moreover, the power-law model failed to converge in the case of the smaller KGPS- $z_{\text {zneb }}$ sample.

Another questionable assumption in the model is that all gas motion is radial with respect to the galaxy centre of mass. We acknowledge that with a more sophisticated parametrization, one might be able to reproduce all of the observed features with a combination of radial and non-radial motions, e.g. orbital or random motions. However, the observations measure line-of-sight components of velocity only, so the distinction between infall, random, and circular/orbital motion based on an ensemble of sightlines would not be evident even with much higher quality data. On the other hand, as long as the line-of-sight component of velocity is slowly varying with $D_{\text {tran }}$ - as expected for typical dark matter halo mass distributions - one can think of the 'inflow' component as a generalized proxy for CGM gas whose motion is dictated by the galaxy potential only, whether that motion is radial, random, circular, or some combination.

To test the robustness of the model fits, we tried fitting the outflow component using constant weighting (rather than inverse variance weighting) across the map; the resulting best-fitting model parameters parameters change by $\lesssim 10$ percent compared to the weighted fit. We also tried fitting the 2D maps of the 'red' and 'blue' halves separately: the power of $\alpha_{\text {ap }}(r)$ (i.e. $\gamma$ ) for inflow is $0.40_{-0.04}^{+0.05}$ on the blue side and $0.71_{-0.03}^{+0.03}$ on the red side, almost different by a factor of 2, while other parameters are consistent within 25 per cent.

Finally, we attempted to fit the observed $\tau_{\text {ap }}$ maps with single component gas distributions, i.e. with infall only or outflow only. The infall model fails to converge because a match to the observations requires an abrupt increase in velocity (by a factor of $\sim 3$ ) and in absorption coefficient (by a factor of $\sim 5$ ) at $r \sim 50 \mathrm{pkpc}$; in any case, as discussed earlier, it is hard to account for $v_{\mathrm{LOS}}>300 \mathrm{~km} \mathrm{~s}^{-1}$ with gravitationally induced infall on to a halo of mass $10^{12} \mathrm{M}_{\odot}$. Moreover, we know unequivocally from the kinematics of strong interstellar absorption lines observed in DTB spectra of the foreground galaxies that outflows dominate the kinematics on scales of at least a few $\mathrm{pkpc}$, and that they have maximum outflow velocities similar to the values of $v_{1}$ in our model outflows.

Fig. 15 shows the best-fitting outflow-only model to the KGPSFull sample. The model includes the effects of Hubble expansion, $v_{\text {out-only }}(r)=v_{\text {out }}(r)+H(z) r$, because the behaviour of the observed map would clearly require a substantial fraction of the outflowing gas to escape the halo, after which its kinematics would be dominated by Hubble expansion. For $D_{\text {tran }} \lesssim 100$ pkpc, the model clearly fails to reproduce either the absorption strength or the kinematics of the central region in order to reproduce the behaviour at larger $D_{\text {tran }}$.

Despite the uncertainty in the parametrization of the model, we argue that the observations require both outflowing and accreting components; this assertion is discussed further in the next section.

\subsubsection{Implications for detailed CGM H I kinematics}

Despite the ability of the model to reproduce the general features of the observed $\tau_{\text {ap }}$ maps, there are some details present in the data that are not successfully captured. Most notable is that the data exhibit contours of constant $\tau_{\text {ap }}$ that change overall shape depending on the contour level, whereas the model contours are relatively smooth and self-similar at different contour levels (Fig. 14) - i.e. the model lacks kinematic structure within $D_{\text {tran }}<100 \mathrm{pkpc}$ and $\left|v_{\mathrm{LOS}}\right|<600 \mathrm{~km} \mathrm{~s}^{-1}$, especially the observed compression between 50 and $100 \mathrm{pkpc}$ that characterizes the observed map. Some of the discrepancy could be caused by the relatively small number of sightlines that comprise the measurements on small $D_{\text {tran }}$ scales, with the correspondingly larger sample variance, and the lack of non-radial gas motion in the model. However, very similar structure at $D_{\text {tran }}<100$ pkpc is observed at both positive and negative $v_{\text {LOS }}$ in the unfolded maps in Fig. 7 , suggesting that the structure is likely to be real.

The rightmost panels of Fig. 14 show the residuals between the observed KGPS-Full maps and the corresponding best-fitting model. These illustrate that the model systematically underpredicts the apparent optical depth at small $D_{\text {tran }}$, particularly for $v_{\text {LOS }} \gtrsim$ $200 \mathrm{~km} \mathrm{~s}^{-1}$, suggesting that a single, smoothly varying outflowing component whose velocity depends only on $r$ is an oversimplification of the true situation. From high-resolution QSO spectra, it is common to observe complexes of absorption systems at small galactocentric impact parameters, with a wide range of $N_{\mathrm{HI}}$ and $v_{\text {LOS }}$ (e.g. Rudie et al. 2012a), with many pixels reaching zero intensity. Disentangling these complexes often requires measurement of the higher Lyman series lines even in spectra with resolution $\lesssim 10 \mathrm{~km} \mathrm{~s}^{-1}$ and very high $\mathrm{S} / \mathrm{N}$; it is therefore unsurprising that a simple model fails to capture 


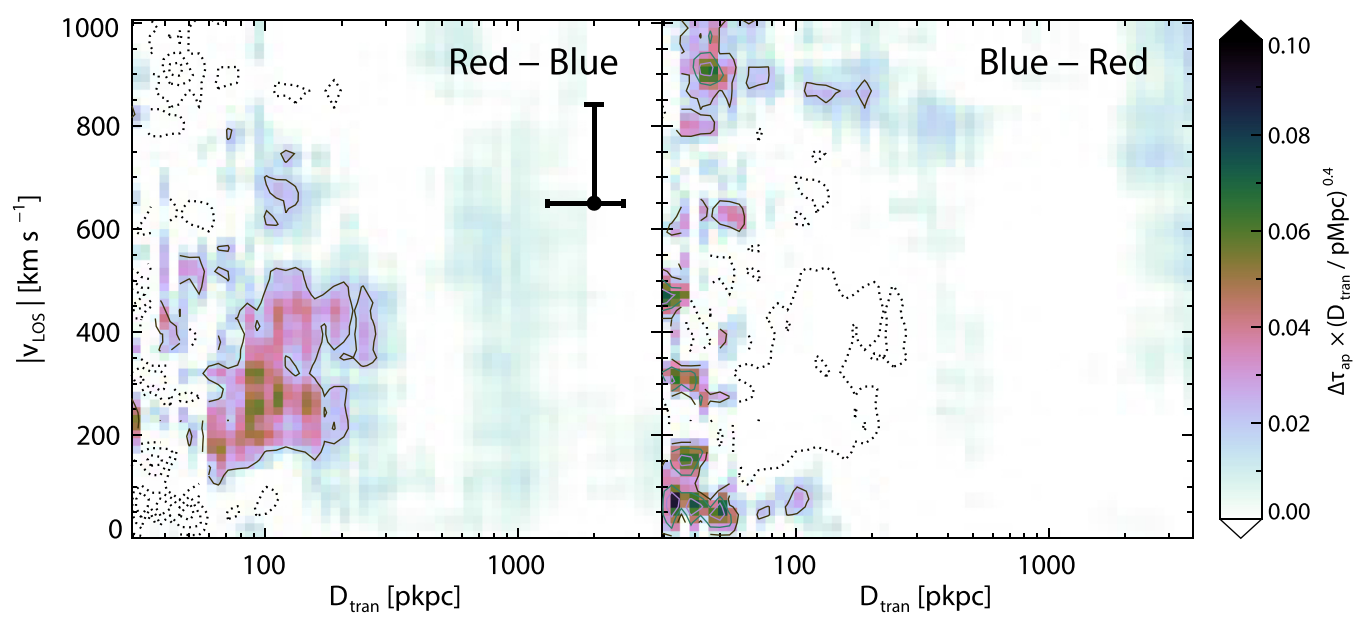

Figure 16. Same as the $\tau_{\text {ap }} \times D_{\text {tran }}^{0.4}$ map in Fig. 8, except that instead of averaging the blue and red sides, the left (right) map is the subtraction of the blue (red) side from the red (blue) side. Significant asymmetry can be seen at $D_{\text {tran }} \simeq 100 \mathrm{pkpc}$. Again, the solid (dotted) contours represent positive (negative) values. The excess blueshifted absorption at $D_{\text {tran }} \lesssim 50 \mathrm{pkpc}$ could be due to sample variation, while the excess redshifted absorption with $D_{\text {tran }}=50-200$ pkpc is likely real

the details. Nevertheless, the rapid fall-off in the maximum $v_{\mathrm{LOS}}$ at relatively high $\tau_{\text {ap }}$ over the range $D_{\text {tran }} \simeq 50-100 \mathrm{pkpc}$, and the flattening of the profile out to $D_{\text {tran }} \simeq 200-300$ pkpc, are not present in our fiducial model or the alternatives discussed in the previous section.

As discussed in Section 4.2.3, a power-law parametrization of the outflow component provides a fit to the $\tau_{\text {ap }}$ map (at least, for the KGPS-Full version) with similar $\chi^{2}$, and allows for a more rapid decrease in $v_{\text {out }}(r)$ with radius compared to the ballistic model with no mass loading. However, it still cannot account for the changing contour shapes of $\tau_{\text {ap }}$ present in the data. Better matches might be obtained by treating high $\tau_{\text {ap }}$ in the outflow component separately from the remainder, allowing it to experience more rapid deceleration at $D_{\text {tran }} \sim 50 \mathrm{pkpc}$ than more diffuse gas, or by a rapid transition from high to low- $N_{\mathrm{H}}$ absorbers for fast-outflowing gas. Both scenarios are consistent with the results of Rudie et al. (2012a), in which absorbers with $N_{\mathrm{HI}}>10^{14.5} \mathrm{~cm}^{-2}$ were found to occupy a smaller range in $v_{\mathrm{LOS}}$ than absorbers with $N_{\mathrm{HI}}<10^{14.5} \mathrm{~cm}^{-2}$ at $D_{\text {tran }} \lesssim 2 \mathrm{pMpc}$.

At 50 pkpc $\lesssim D_{\text {tran }} \lesssim 100 \mathrm{pkpc}$, where the $\tau_{\text {ap }}$ profile is at its narrowest, the line profile (i.e. the distribution of $v_{\text {LOS }}$ at a given $\left.D_{\text {tran }}\right)$ is consistent with the effective velocity resolution of the map $\left(\sim 190 \mathrm{~km} \mathrm{~s}^{-1}\right)$, and changes very little out to $D_{\text {tran }} \sim 100-200 \mathrm{pkpc}$. As best seen in the bottom panels of Fig. 14, beyond $D_{\text {tran }} \sim 100 \mathrm{pkpc}$, while the optical depth continues to decrease roughly as $D_{\text {tran }}^{-0.4}$, the range of $v_{\text {LOS }}$ begins to increase again, by an amount that depends on the contour level. The best-fitting radial and kinematic profiles shown in Fig. 13 suggest that the minimum in the line-of-sight velocity field at $D_{\text {tran }} \sim 100-200$ pkpc may mark a caustic where outflows and infall both reach minimum $v_{\text {LOS }}$, perhaps with opposite sign. The location of this feature also corresponds to the clear change in slope of the relationship between $D_{\text {tran }}$ and $W_{\lambda}(\operatorname{Ly} \alpha)$ (Fig. 6), and is just beyond the expected virial radius given a halo mass of $M_{\mathrm{h}}=10^{12} \mathrm{M}_{\odot}$. Since the escape velocity at 100 pkpc for such a halo is $v_{\text {esc }} \simeq 440 \mathrm{~km} \mathrm{~s}^{-1}$, we expect that most of the neutral $\mathrm{H}$ at $r \lesssim 100$ pkpc remains bound to the central galaxy.

\subsection{Velocity asymmetry and emission filling}

One of the more puzzling features of the $2 \mathrm{D}$ maps of $\tau_{\text {ap }}$ - evident in Fig. 7 - is the apparent asymmetry of $\tau_{\text {ap }}$ with respect to $v_{\text {LOS }}$. In the KGPS-Full sample, for $D_{\text {tran }}$ between $\sim 70$ and $150 \mathrm{pkpc}$, the integrated $\tau_{\text {ap }}$ for $v_{\mathrm{LOS}}>0$ is $>1.5$ times that of the corresponding value for $v_{\mathrm{LOS}}<0$, meaning that redshifted Ly $\alpha$ absorption is stronger than blueshifted Ly $\alpha$ absorption. The asymmetry can also be seen in Fig. 5: for the composite $\operatorname{Ly} \alpha$ spectrum for the bin with $80 \leq D_{\text {tran }} / \mathrm{pkpc} \leq 160$, although the maximum depth in the absorption profile occurs at a velocity consistent with $v_{\mathrm{LOS}}=0$, there is a red wing extending to $v_{\mathrm{LOS}} \sim+500 \mathrm{~km} \mathrm{~s}^{-1}$, whereas the blue absorption wing reaches only $v_{\text {LOS }} \sim-300 \mathrm{~km} \mathrm{~s}^{-1}$. Similar asymmetry is also present for the KGPS- $z_{\text {neb }}$ sample, suggesting that residual systematic redshift errors are not likely to be the principal cause.

Fig. 16 shows the result of subtracting the blue (red) component of the $\tau_{\text {ap }} \times D_{\text {tran }}^{0.4}$ map from the red (blue) side for the KGPS-Full sample. The most prominent residual appears at $50 \lesssim D_{\text {tran }} / \mathrm{pkpc}$ $\lesssim 200$ and $200 \lesssim\left|v_{\mathrm{LOS}}\right| / \mathrm{km} \mathrm{s}^{-1} \lesssim 500$; residuals resulting from differencing of the KGPS- $z_{\text {neb }}$ sample are similar. Although the residual has modest $\mathrm{SNR}$ ( $\simeq 2$ per $\AA$ ), it extends over a contiguous region larger than the effective resolution of the map, and is therefore significant. There is a fractionally less-significant excess of blueshifted Ly $\alpha$ absorption at $D_{\text {tran }} \lesssim 50$ pkpc (better shown in the right-hand panel of Fig. 16), apparently extending over the full range $0 \leq\left|v_{\mathrm{LOS}}\right| / \mathrm{km} \mathrm{s}^{-1} \leq 1000$ that could be attributable to the relatively small number of galaxy pairs at small separations (i.e. to sample variance). It is harder to dismiss the excess redshifted absorption in the left-hand panel of Fig. 16, which is based on a 10times larger sample $(\simeq 1000)$ of galaxy pairs. On the other hand, there is no significant asymmetry in the Ly $\alpha$ absorption profiles beyond $D_{\text {tran }} \sim 200$ pkpc, where the SNR of the map is high.

If the observed asymmetry is of astrophysical origin, it remains to be explained. We considered possible causes of the apparent excess redshifted Ly $\alpha$ absorption, noting that it is confined to the foreground galaxy CGM within $D_{\text {tran }} \simeq 200$ pkpc: possibilities include observational selection effects or biases, which might have been introduced by particular properties favoured among the foreground or background galaxies that were observed successfully (e.g. compactness, dust attenuation, orientation, etc.). We could not envision a plausible scenario that could explain the asymmetry in the absorption profile.

We also considered the real temporal difference between the time light from the background galaxy passes through the 'far' and 'near' 
sides of the foreground galaxy CGM, but the relevant time-scale would be very short, $\Delta t \lesssim 400 \mathrm{pkpc} / c \sim 10^{5} \mathrm{yr}$. As above, it is hard to explain why any time-dependent effect would systematically bias the kinematics of the absorbing gas.

It is now well-known that Ly $\alpha$ emission 'haloes' are a generic property of SF galaxies at high redshift (e.g. Steidel et al. 2011; Wisotzki et al. 2016; Erb, Steidel \& Chen 2018) and that they extend to projected distances $\sim 5-10$ times larger than their UV continuum light. Of the possible explanations, we find the least implausible to be that the Ly $\alpha$ absorption profile has been altered by Ly $\alpha$ emission contamination within the spectroscopic aperture used to record the background galaxy spectra. Most surveys of intervening absorption lines have used bright background point sources, such as QSOs, in which case this possible source of contamination can ordinarily be neglected. One usually thinks of Ly $\alpha$ absorption systems in terms of equivalent width $W_{\lambda}(\operatorname{Ly} \alpha)$ or column density $N_{\mathrm{HI}}$, but one can also think of a $\operatorname{Ly} \alpha$ absorption feature at $z=z_{\mathrm{fg}}$ as a record of the flux removed from the beam of the background source at $z_{\mathrm{bg}}$, scattered out of the line of sight by H I in the CGM of a foreground galaxy. As an example, for an observation of a $m=18$ QSO at $z_{\mathrm{bg}}$ whose sightline passes near to a typical $z_{\mathrm{fg}}=2.2$ galaxy and produces an absorption feature with rest-frame $W_{\lambda}(\operatorname{Ly} \alpha)=1.0 \AA$ at $z_{\mathrm{fg}}$, the flux removed from the QSO spectrum would be $\simeq 6 \times 10^{-16} \mathrm{erg} \mathrm{s}^{-1} \mathrm{~cm}^{-2}$, which is large even in comparison to the total Ly $\alpha$ flux from the galaxy (Steidel et al. 2011), and hundreds of times larger than the Ly $\alpha$ flux likely to be collected by a slit located 5-10 arcsec away from the $z_{\text {fg }}$ galaxy. For a typical $m=25$ background galaxy, on the other hand, the same absorption line equivalent width would correspond to a flux smaller by a factor of $\sim 630$, or $\simeq 10^{-18} \mathrm{erg} \mathrm{s}^{-1} \mathrm{~cm}^{-2}$, i.e. approaching the flux expected from the low surface brightness Ly $\alpha$ emission halo of the foreground galaxy. In the latter case, the presence of the Ly $\alpha$ emission could have a measurable effect on the apparent absorption strength if the velocity ranges overlap.

To be more quantitative, we estimated the flux of Ly $\alpha$ emission captured within the aperture used to obtain background galaxy spectra using the average Ly $\alpha$ halo observed by Steidel et al. (2011; hereafter S2011) for a sample of SF galaxies with similar properties to those in the larger KBSS sample, but with $\langle z\rangle=2.65$. The mean Ly $\alpha$ surface brightness profile was found to be reasonably welldescribed by an exponential, $S(b)=C_{1} \exp \left(-b / b_{0}\right)$, where $b=D_{\text {tran }}$, $C_{1}=2.4 \times 10^{-18} \mathrm{erg} \mathrm{s}^{-1} \mathrm{~cm}^{-2} \operatorname{arcsec}^{-2}$, and $b_{0}=25 \mathrm{pkpc}$. The mean halo is detected down to a surface brightness limit of $S \simeq 10^{-19}$ $\mathrm{erg} \mathrm{s}^{-1} \mathrm{~cm}^{-2} \operatorname{arcsec}^{-2}$ at $D_{\text {tran }} \sim 80 \mathrm{pkpc}$.

By assuming that the intrinsic Ly $\alpha$ luminosity and scale length of the mean Ly $\alpha$ halo of the KGPS foreground galaxies are the same as those of the S2011 sample, we applied the redshift-dependent surface brightness correction to move from $\langle z\rangle=2.65$ to $\langle z\rangle=2.25$, and extrapolated the Ly $\alpha$ surface brightness profile to $D_{\text {tran }}=100 \mathrm{pkpc}$. The predicted Ly $\alpha$ surface brightness would be $\sim 7.2 \times 10^{-20}$ erg $\mathrm{s}^{-1} \mathrm{~cm}^{-2} \operatorname{arcsec}^{2}$. Taking the typical extraction aperture for the LRIS spectra to be of angular size $1.35 \operatorname{arcsec} \times 1.2 \operatorname{arcsec}\left(\sim 1.6 \operatorname{arcsec}^{2}\right)$, the integrated Ly $\alpha$ emission flux within the slit would be $F_{\mathrm{Ly} \alpha, \mathrm{em}} \sim$ $1.1 \times 10^{-19} \mathrm{erg} \mathrm{s}^{-1} \mathrm{~cm}^{-2}$.

We estimated the mean background galaxy continuum flux density near $4000 \AA$ (i.e. the wavelength of Ly $\alpha$ at $z \sim 2.25$ ) by interpolating the flux density between the photometric $U_{\mathrm{n}}(3520 / 600)$ and $G$ (4730/1100) passbands, with the result $\left\langle m_{\mathrm{AB}}(4000 \AA)\right\rangle \simeq 25.3$, or $\left\langle F_{\nu}\right\rangle \simeq 0.26 \mu \mathrm{Jy}\left(\left\langle F_{\lambda}\right\rangle \simeq 5 \times 10^{-19} \mathrm{erg} \mathrm{s}^{-1} \mathrm{~cm}^{-2} \AA^{-1}\right.$. $)$

The observed Ly $\alpha$ absorption equivalent width at $D_{\text {tran }} \simeq 100 \mathrm{pkpc}$ is $\left\langle W_{\lambda}(\operatorname{Ly} \alpha)_{\text {obs }}\right\rangle \simeq 2.7 \AA$ (assuming $\langle z\rangle=2.25$ ), which removes an average flux from the background galaxy spectrum of $2.7 \times 5 \times$ $10^{-19} \simeq 1.4 \times 10^{-18} \mathrm{erg} \mathrm{s}^{-1} \mathrm{~cm}^{-2}$. The fractional perturbation of

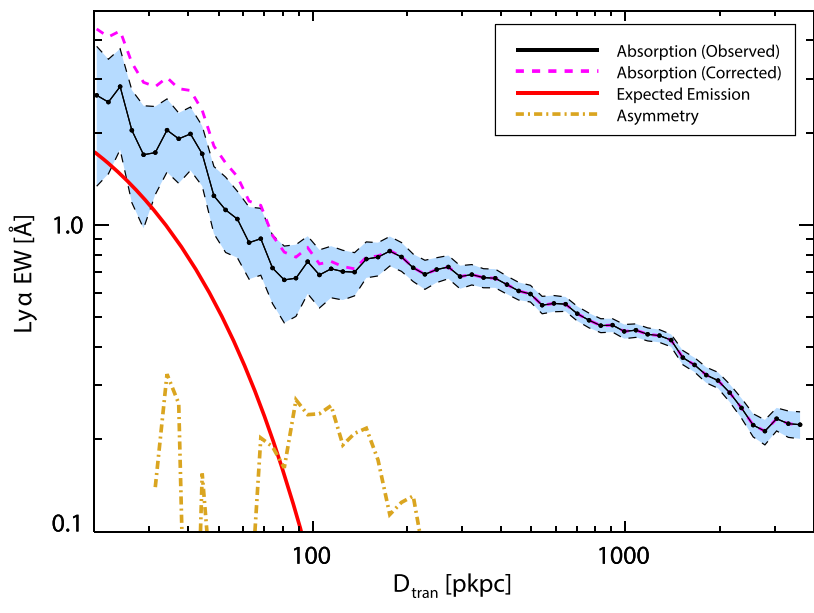

Figure 17. Expected contribution to $W_{\lambda}(\operatorname{Ly} \alpha)$ by Ly $\alpha$ emission surrounding foreground galaxies (red), compared to the observed $W_{\lambda}(\operatorname{Ly} \alpha)$ in absorption (black, same as in Fig. 6). The dashed magenta curve shows the absorption after correction for the estimated contribution from emission filling. The yellow dashed curve shows the fraction of $W_{\lambda}(\operatorname{Ly} \alpha)$ contributed by asymmetry, calculated by integrating the blue/red halves of the absorption profiles spectra within $\left|\Delta v_{\text {LOS }}\right|<700 \mathrm{~km} \mathrm{~s}^{-1}$ and subtracting one from the other.

the total Ly $\alpha$ absorption equivalent width by foreground galaxy Ly $\alpha$ emission is then $1.1 \times 10^{-19} / 1.4 \times 10^{-18} \simeq 0.08$ ( 8 per cent). Using the same arguments, emission filling at $D_{\text {tran }} \simeq 25 \mathrm{pkpc}-$ where the mean Ly $\alpha$ surface brightness is $\sim 20$ times higher (according to S2011) but the absorption rest equivalent width is larger by a factor of only $\simeq 2.5$ (see Fig. 6) - emission would be predicted to affect the observed $W_{\lambda}(\operatorname{Ly} \alpha)$ at the $\gtrsim 50$ percent level. Fig. 17 shows the predicted effect of diffuse emission on the observed $W_{\lambda}(\operatorname{Ly} \alpha)$

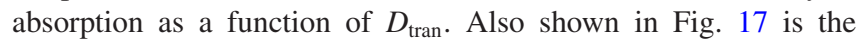
amplitude of the observed asymmetry converted to $\Delta W_{\lambda}(\operatorname{Ly} \alpha)$, the net equivalent width of the $W_{\lambda}(\operatorname{Ly} \alpha)$ residuals shown in Fig. 16.

The average equivalent width of the residual shown in the lefthand panel of Fig. 16 is $\Delta W_{\lambda}(\operatorname{Ly} \alpha) \simeq 0.2 \AA$ for $70 \lesssim D_{\text {tran }} / \mathrm{pkpc}$ $\lesssim 150$, or $\simeq 25-30$ per cent of the total observed $W_{\lambda}($ Ly $\alpha)$ over the same range of $D_{\text {tran }}-$ within a factor of $\sim 3$ of the estimated effect from emission filling, possibly consistent given the uncertainties. However, if the emission was distributed symmetrically in velocity space with respect to the absorption, its effect would mostly likely have remained unrecognized. The asymmetry in Ly $\alpha$ absorption at $D_{\text {tran }} \sim 100 \mathrm{pkpc}$, if attributed to emission filling, would require that the bulk of Ly $\alpha$ emission must be blueshifted by $\Delta v_{\text {LOS }} \sim$ $200-400 \mathrm{~km} \mathrm{~s}^{-1}$ with respect to the foreground galaxy systemic redshift. This is opposite to what is typically observed for Ly $\alpha$ emission in DTB galaxy spectra, in which the dominant component of Ly $\alpha$ emission is redshifted by several hundred $\mathrm{km} \mathrm{s}^{-1}$ for the galaxies in the KBSS sample (e.g. see the top and bottom panels of Fig. 2.) In this context, it is possibly relevant that the excess emission (if that is indeed the cause) falls in the range of $D_{\text {tran }}$ where our kinematic modelling (Section 4.2) suggested a transition between outflow-dominated and accretion-dominated flows. If the velocity field at $D_{\text {tran }} \simeq 100 \mathrm{pkpc}$ was dominated by inflows, scattering of Ly $\alpha$ photons in the observer's direction would tend to be blueshifted (e.g. Faucher-Giguère et al. 2010; Dijkstra 2014).

Also qualitatively (but perhaps not quantitatively) consistent with this picture is that the asymmetry on smaller transverse scales ( $D_{\text {tran }}$ $\lesssim 40$ pkpc) has the opposite sign, i.e. the absorption is stronger on the blueshifted side of $v_{\text {sys }}$, which might be attributable to excess 
redshifted emission, as for typical DTB spectra. However, as shown in Fig. 17, in order to be consistent with the expected effect of emission filling at small $D_{\text {tran }}$, the kinematic asymmetry of the Ly $\alpha$ emission would need to comprise only a fraction of the total emission, since the observed net effect on $W_{\lambda}(\operatorname{Ly} \alpha)$ from the asymmetry is only $\sim 10-15$ per cent. On the other hand, recent observations using the Keck Cosmic Web Imager (KCWI; Morrissey et al. 2018) for a subset of the KBSS galaxy sample suggests that the ratio between the blueshifted and redshifted components of Ly $\alpha$ emission from the CGM increases with projected galactocentric distance (Erb et al. 2018; Chen et al., in preparation), and approaches $1: 1$ by $r \sim 50$ pkpc; unfortunately, the observations of individual galaxies with KCWI (or MUSE) are not yet sensitive enough to evaluate at $D_{\text {tran }}>50$ pkpc. ${ }^{15}$

An obvious observational test of the hypothesis that emission filling significantly alters the strength of $W_{\lambda}(\operatorname{Ly} \alpha)$ measured along background galaxy sightlines could be made using QSO sightlines, since QSOs are at least $\sim 6$ mag brighter than the typical $z \sim$ 2.5 background galaxy, so that foreground galaxy Ly $\alpha$ emission is expected to be negligible compared to the absorbed flux from the background source. Unfortunately, the sample size of QSOgalaxy pairs for $z_{\mathrm{fg}}>2$ is very small in comparison. We examined the velocity distribution of $\mathrm{HI}$ for QSO sightlines within $D_{\text {tran }} \simeq$ 200 pkpc of KBSS galaxies in Rudie et al. (2012a, 2019). In the range of $50<D_{\text {tran }} / \mathrm{pkpc}<200$, there are slightly more redshifted absorption components by number, but the excess is not statistically significant. When the high-resolution QSO spectra are analysed in the same way as the galaxy spectra by smoothing to reduce the spectral resolution and averaging the spectral regions near Ly $\alpha$ at the redshift of foreground galaxies - as done by Turner et al. (2014) (see Fig. 6) - $W_{\lambda}($ Ly $\alpha)$ is larger by $\simeq 50$ per cent for the QSO sightlines for the bin at $D_{\text {tran }} \simeq 90$ pkpc compared to the observed $W_{\lambda}(\operatorname{Ly} \alpha)$ for the KGPS sightlines. Though the two measurements are still consistent with one another at the $\sim 2 \sigma$ level, the difference is in the direction expected if the KGPS sample has been affected by Ly $\alpha$ emission. At smaller $D_{\text {tran }}$, where the expected effect of emission filling in the galaxy sightlines is larger, the QSO sightline samples are too small to provide a meaningful comparison (e.g. there are no QSO-galaxy pairs with $D_{\text {tran }}<50$ pkpc in the KBSS sample used by Turner et al. 2014.)

In any case - whether or not it is responsible for the asymmetry in the current data - it is probably important to account for the effects of emission filling on $W_{\lambda}(\operatorname{Ly} \alpha)$ in absorption, as a function of $D_{\text {tran }}$, which will depend on the relative brightness of the foreground and background sources. When we apply our estimate from above, the corrected $W_{\lambda}($ Ly $\alpha)$ versus $D_{\text {tran }}$ (Fig. 17) accentuates the existence of two distinct 'zones' in the behaviour of Ly $\alpha$ absorption around KBSS galaxies: the inner zone, at $D_{\text {tran }} \lesssim 100$ pkpc, where $W_{\lambda}($ Ly $\alpha) \propto$ $D_{\text {tran }}^{-1.1}$, and an outer zone with $100 \lesssim D_{\text {tran }} / \mathrm{pkpc} \lesssim 300 \mathrm{kpc}$, over which $W_{\lambda}(\operatorname{Ly} \alpha)$ remains remarkably flat.

\subsection{Baryon escape}

A significant fraction of gas associated with galaxy-scale outflows is expected to be retained by relatively massive galaxies. Even if some gas does manage to escape to beyond the halo virial radius, theoretical expectations are that, for galaxies with halo masses $\simeq 10^{12} \mathrm{M}_{\odot}$ at $z \gtrsim 2$, most will eventually be re-accreted by the galaxy through a process known as 'recycling' (e.g. Oppenheimer et al. 2010; Muratov

\footnotetext{
${ }^{15}$ Over the range of $D_{\text {tran }}$ in common, the mean Ly $\alpha$ halo in the KCWI data are consistent in both shape and intensity with the mean Ly $\alpha$ halo presented by S2011.
}

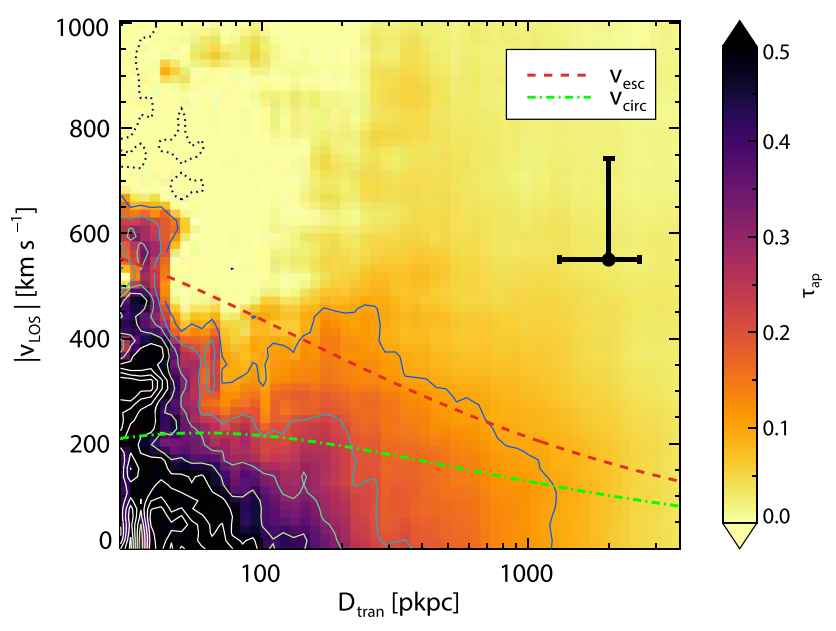

Figure 18. Same as the $\tau_{\text {ap }}$ map in Fig. 8, with the radial dependence of the escape velocity ( $v_{\text {esc }}$, red dashed) and circular velocity ( $v_{\text {circ }}$, green dashdotted) for an NFW halo with $M_{\mathrm{h}}=10^{12} \mathrm{M}_{\odot}$ superposed (assuming that $D_{\text {tran }}$ is equivalent to the galactocentric distance $r$.).

et al. 2015). It is of interest to ask whether the observed ensemble kinematics of circumgalactic HI we have presented in previous sections suggests the presence of neutral gas capable of escaping the potential well of the central galaxy. The most straightforward indicator would be significant absorption components of the CGM with $v_{\mathrm{LOS}}\left(D_{\text {tran }}\right)>v_{\text {esc }}(r)$ where both are measured relative to the central galaxy systemic redshift (e.g. Adelberger et al. 2005b; Rudie et al. 2019.)

Fig. 18 reproduces the folded map of $\tau_{\text {ap }}$, with curves denoting the $3 \mathrm{D}$ escape velocity $\left(v_{\text {esc }}\right)$ for a $M_{\mathrm{h}}=10^{12} \mathrm{M}_{\odot} \mathrm{NFW}$ halo: since the map shows only projected distances and line-of-sight velocities, the actual galactocentric radius and $3 \mathrm{D}$ space velocities of gas may be greater than $D_{\text {tran }}$ and $v_{\text {LOS }}$, respectively. Considering only gravity as in Section 4.2, any gas with $v_{\text {LOS }}>v_{\text {esc }}$ would be capable of escaping the halo. Conversely, gas with $v_{\mathrm{LOS}}<v_{\mathrm{esc}}$ is not guaranteed to be bound to the halo, but the chance of escape rapidly decreases as $\left|v_{\text {LOS }}\right|$ $\rightarrow 0$. Clearly, judged on this basis, most of the relatively high- $\tau_{\text {ap }} \mathrm{H} \mathrm{I}$ within $D_{\text {tran }} \lesssim 100 \mathrm{pkpc}$ is unlikely to escape the galaxy potential.

Nevertheless, it remains likely that some gas does escape, given the presence of significant $\tau_{\text {ap }}$ with $v_{\text {LOS }}$ close to or exceeding $v_{\text {esc }}$ It is also likely that $\mathrm{HI}$ is not the best tracer of the fastest moving gas, particularly at large galactocentric distances, based on both observations of high-ionization metals in nearby and high-redshift starburst galaxies (e.g. Strickland et al. 2004; Strickland \& Heckman 2009; Turner et al. 2015; Rudie et al. 2019) and on simulations such as those presented in Section 4.1.

\subsection{Redshift-space distortions}

In order to highlight the effects of peculiar velocities on the observations, we resampled the $\tau_{\text {ap }}$ map in the line-of-sight direction, assuming that $v_{\text {LOS }}$ is due entirely to Hubble expansion, i.e. that $D_{\text {LOS }}=v_{\text {LOS }} / H(z)$. Fig. 19 shows the re-sampled $\tau_{\text {ap }}$ map of the KGPS-Full sample with matching $D_{\text {Los }}$ and $D_{\text {tran }}$ axes. Regions with $D>100$ pkpc closely resemble similar results compiled using QSO sightlines from KBSS (Rakic et al. 2012; Rudie et al. 2012a; Turner et al. 2014).

Fig. 20 shows direct comparisons of $\tau_{\text {ap }}$ profiles extracted along the two axes (line of sight and transverse to the line of sight) for 


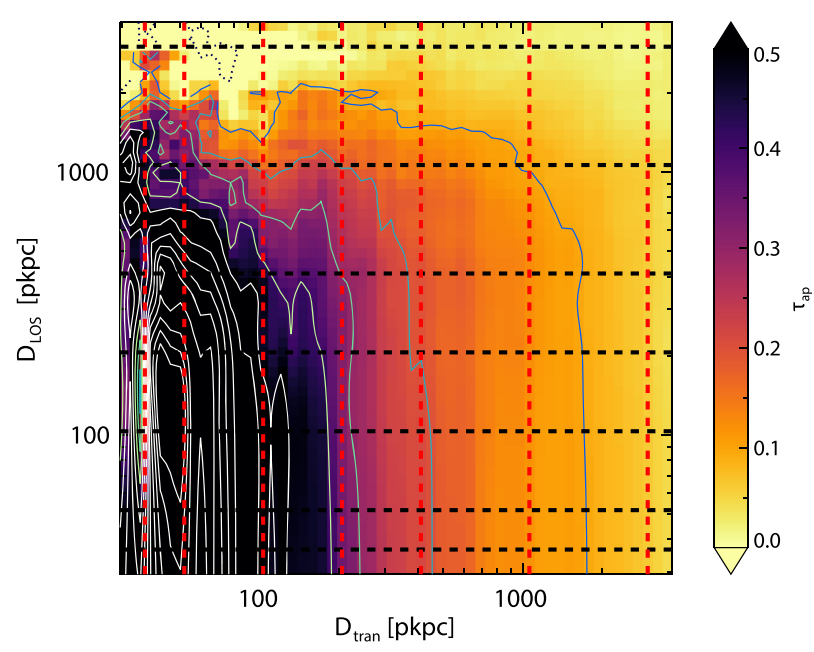

Figure 19. Same as the top-left plot of Fig. 8, except here the $y$-axis has been converted to line-of-sight distance assuming that $v_{\text {LOS }}$ is entirely due to Hubble expansion. The two axes have been adjusted so that any departures from symmetry indicate the presence of peculiar motions of gas with respect to the Hubble flow. The red vertical dashed lines and black horizontal dashed lines correspond to the locations of extracted profiles in Fig. 20.

seven representative distances, randomly chosen from the figure. In the absence of peculiar velocities and redshift errors, the $\tau_{\text {ap }}$ profiles at fixed $D_{\text {Los }}$ and $D_{\text {tran }}$ would be identical. In reality, the $D_{\text {LOS }}$ and $D_{\text {tran }}$ profiles differ significantly: e.g. at $D \lesssim 500 \mathrm{pkpc}$, the $D_{\text {tran }}$ cuts have similar shapes, while the corresponding $D_{\mathrm{LOS}}$ cuts on the same scales vary considerably. Most obvious is the 'finger of God' elongation, known as the 'Kaiser effect' (Kaiser 1987), along the line of sight due to peculiar velocities of gas with $D_{\text {tran }} \lesssim 100 \mathrm{pkpc}$. However, there is also a more subtle signature of infall in Fig. 19 that is evident only at the lowest two contours in $\tau_{\text {ap }}$, manifesting most clearly as a compression in the $D_{\text {LOS }}$ direction between $D_{\text {tran }} \simeq 100$ and $D_{\text {tran }} \simeq 50 \mathrm{pkpc}$ as also noted previously.

At $D \gtrsim 500$ pkpc (upper-right corner of Fig. 19), the $D_{\text {tran }}$ and $D_{\text {Los }}$ cuts begin to match, indicating diminishing redshift-space distortion.

In addition to the KBSS-based studies mentioned above, there have been a number of other studies, focused on establishing the crosscorrelation between 'absorbers' along QSO sightlines and galaxies in the surrounding volumes at $z<1$ (e.g. Chen et al. 2005; RyanWeber 2006; Chen \& Mulchaey 2009; Tejos et al. 2014), $0.7 \lesssim z \lesssim$ 1.5 (Shone et al. 2010), and $z \simeq 2-3$ (Adelberger et al. 2003, 2005b; Tummuangpak et al. 2014; Bielby et al. 2017). Care must be exercised in comparing the results of these studies with those presented in this work, for two reasons: first, the aforementioned studies have cast the results in terms of auto- and cross-correlation functions, in co-moving coordinates, so that all length-scales in this work must be suitably $\operatorname{adjusted}^{16}$; secondly, most of these studies were not well-suited to measuring galaxy-gas correlations on scales smaller than $\sim 1 \mathrm{cMpc}$ ( $\sim 300$ pkpc at $\langle z\rangle=2.2$ ) either due to redshift errors ( $z>2$ studies), paucity of absorber-galaxy pairs, or both. We note that the redshift space distortion in the KGPS map - both the 'finger of God' at $D_{\text {tran }}$ $\lesssim 50$ pkpc and the 'compression' on scales of $50 \lesssim D_{\text {tran }} \lesssim 200$ pkpc,

\footnotetext{
${ }^{16}$ In comoving coordinates, the transverse scale for the 2D map in (e.g.) Fig. 7 would need to be multiplied by a factor of $\simeq 3$. 2 for direct comparison, i.e. our measurements extend to transverse a transverse scale of $D_{\text {tran,com }} \simeq 12.8 h_{70}^{-1}$ cMpc.
}

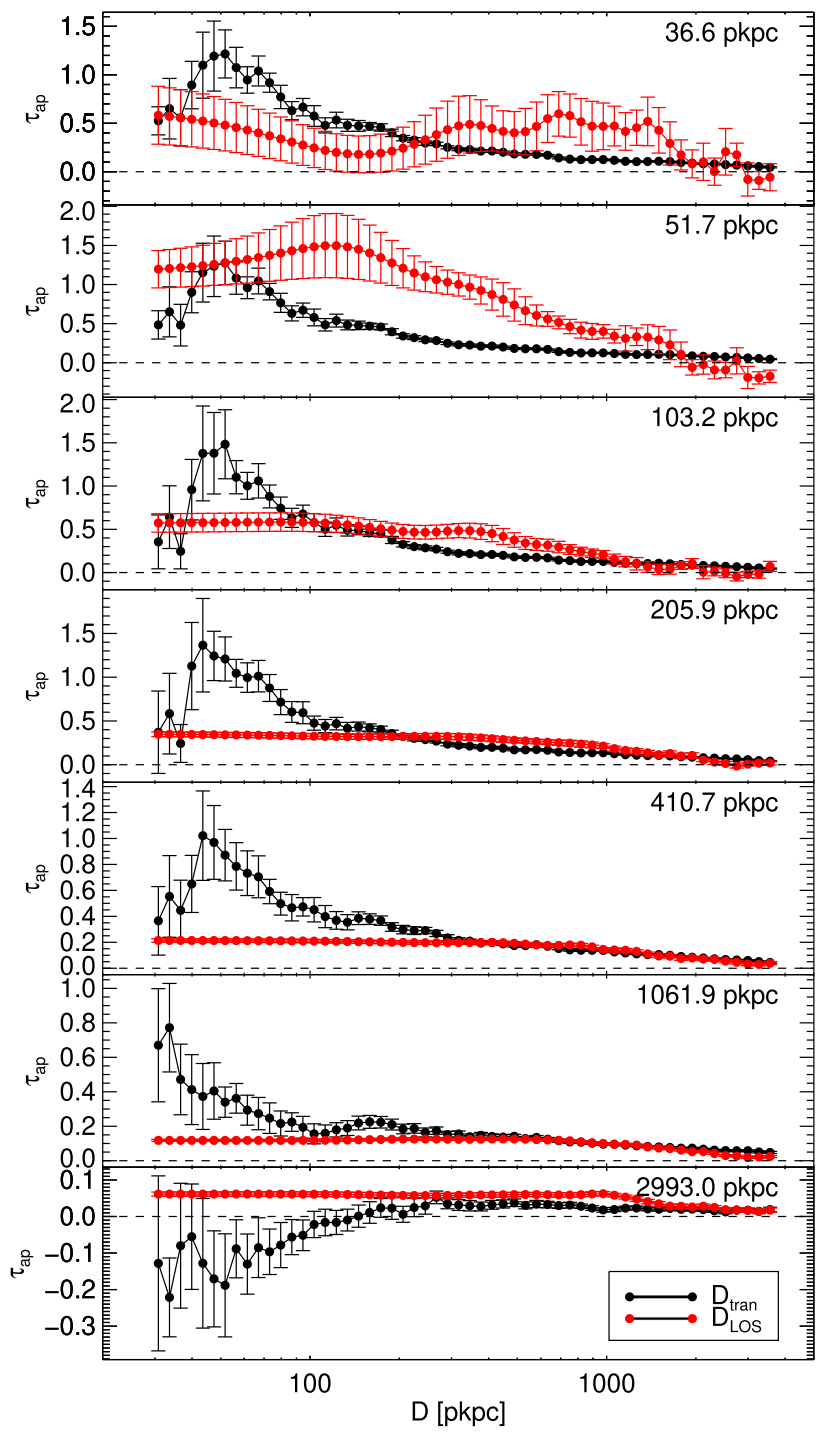

Figure 20. Comparison of the extracted $\tau_{\text {ap }}$ profiles (see Fig. 19) in the $D_{\text {tran }}$ (black) and $D_{\mathrm{LOS}}$ (red) directions. Numbers on the top-right corner of the plots are the distances in pkpc to the centre of the galaxy in the direction orthogonal to that over which the profile is extracted.

would probably have gone unrecognized. Otherwise, on scales larger than $\sim 1 \mathrm{cMpc}$, there is reasonable agreement: for example, one of the conclusions of Tejos et al. (2014) is that there is little evidence for either infall or outflows of gas with peculiar velocities larger than $\sim 120 \mathrm{~km} \mathrm{~s}^{-1}$; our results are in agreement - recall that we found that infall velocities of $\sim 100 \mathrm{~km} \mathrm{~s}^{-1}$ gave the best fits for our simple model. However, there are much larger peculiar velocities acting on scales smaller than $\simeq 150$ pkpc $(0.5 \mathrm{cMpc})$, within the CGM.

Finally, we note that the values of $\tau_{\text {ap }}$ for the $D_{\text {tran }}$ profile evaluated at $D_{\mathrm{LOS}}=2993 \mathrm{pkpc}$ (black points and curve in the bottom panel of Fig. 20) are consistently negative for $D_{\text {tran }} \lesssim 100 \mathrm{pkpc}$. Because most of the points are correlated and each is individually consistent with $\tau_{\text {ap }}=0$, we believe the most likely culprits are sample variance and continuum uncertainties exacerbated by the relatively small sample size. Meanwhile, as discussed in Section 4.3, it is possible that Ly $\alpha$ emission from the CGM of the foreground galaxy has a significant effect on the measured strength of Ly $\alpha$ absorption in the spectrum of faint background continuum sources, and that the magnitude of 
the effect would be largest at small $D_{\text {tran }}$. Scattered Ly $\alpha$ emission in down-the-barrel spectra of the galaxies tends to be dominated by a redshifted component, and it is not unusual for the red wing of Ly $\alpha$ to extend well beyond $v_{\mathrm{LOS}} \sim 600 \mathrm{~km} \mathrm{~s}^{-1}$; if the Ly $\alpha$ emission strength exceeds the flux removed by absorption against the continuum of the background source, it is possible in principle to have 'negative' net Ly $\alpha$ absorption.

\section{SUMMARY}

In this paper, we assembled 2862 spectroscopically identified galaxies from KBSS $(1.9 \lesssim z \lesssim 3.3 ;\langle z\rangle=2.51)$ into $\sim 200000$ unique angular pairs of physically unrelated galaxies; we then used the spectra of the background galaxies to probe the HI content of the $\mathrm{CGM} / \mathrm{IGM}$ of the foreground galaxies as a function of projected physical distance over the range of $30 \lesssim D_{\text {tran }} /$ pkpc $\lesssim 4000$.

To maximize the utility of composite spectra for mapping the strength and kinematics of Ly $\alpha$ absorption surrounding galaxies, we used the $\simeq 45$ per cent of galaxies with precise and accurate nebular emission-line measurements to re-calibrate the relationship between the galaxy systemic redshift from $z_{\text {neb }}$ and redshifts measured using spectral features in the rest-frame far-UV, $z_{\mathrm{Ly} \alpha}$ and/or $z_{\mathrm{IS}}$, which are biased by the effects of outflowing gas. We created composite spectra, stacked in bins of $D_{\text {tran }}$, of background galaxy spectra shifted to the rest frame of the corresponding foreground galaxy in each pair. The very large number of distinct galaxy-galaxy pairs allowed us to construct a well-sampled ensemble map of neutral $\mathrm{H}$ surrounding the average foreground galaxy in the sample. In particular, the improved sampling within $D_{\text {tran }} \lesssim 100$ pkpc is crucial in probing the effects of galaxy-scale outflows on the H I kinematics.

We compared the observed Ly $\alpha$ map with cosmological zoom-in simulations and with a simple analytical model of outflows and infall surrounding a galaxy hosted by a dark matter halo of mass $M_{\mathrm{h}} \simeq 10^{12}$ $\mathrm{M}_{\odot}$. The principal results are summarized below:

(i) The Ly $\alpha$ equivalent width as a function of impact parameter $D_{\text {tran }}$ can be approximated as a power law, $\mathrm{W}_{\lambda}(\mathrm{Ly} \alpha) \propto D_{\text {tran }}^{-0.4}$ over the full range observed, but there are at least three distinct impact parameter zones for the run of $W_{\lambda}(\operatorname{Ly} \alpha)$ versus $D_{\text {tran }}: D_{\text {tran }}<100$ pkpc (slope $\simeq-1.0), 100<D_{\text {tran }} /$ pkpc $\lesssim 300$ (slope $\left.\simeq 0\right)$, and 300 $\lesssim D_{\text {tran }} /$ pkpc $\lesssim 2000$ (slope $\left.\simeq-0.5\right)($ Section 3.1)

(ii) The 2D map of apparent Ly $\alpha$ optical depth $\tau_{\text {ap }}$ (Figs 7 and 8) in $v_{\mathrm{LOS}}-D_{\text {tran }}$ space exhibits a dense 'core' at $\left|v_{\mathrm{LOS}}\right|<500 \mathrm{~km} \mathrm{~s}^{-1}$ and $D_{\text {tran }}<100 \mathrm{pkpc}$ that transitions to a diffuse component that becomes broader with increasing $D_{\text {tran }}$. The maps using the full KGPS sample and the sub-sample for which nebular redshifts are available for the foreground galaxy show consistent features. (Section 3.2)

(iii) Comparison of the $\tau_{\text {ap }}$ map with the projected $N_{\mathrm{H} \text { I }}$ map of a simulation with similar halo mass to the observed sample from the FIRE project shows that the dense 'core', and the outer 'envelope' match remarkably well in both $v_{\text {LOS }}$ and $D_{\text {tran }}$. (Section 4.1)

(iv) A simple, two-component analytical model with radial inflow and outflow can reproduce the general features of the observed 2D $\tau_{\text {ap }}$ map; however, the model fails to fit abrupt features in the Ly $\alpha$ absorption kinematics at particular values of $D_{\text {tran }}$, which clearly indicate a level of complexity that is not captured by the adopted model parametrization. (Section 4.2)

(v) The $\tau_{\text {ap }}$ map exhibits significant asymmetry in velocity relative to the galaxy systemic redshifts, the strongest of which is at projected distances $50 \lesssim D_{\text {tran }} /$ pkpc $\lesssim 200$ and $200 \mathrm{~km} \mathrm{~s}^{-1} \lesssim\left|\mathrm{v}_{\text {LOS }}\right| \lesssim$ $500 \mathrm{~km} \mathrm{~s}^{-1}$. The asymmetry is significant, and is unlikely to be explained by unaccounted-for systematic errors in galaxy redshifts.
We suggest that the most plausible explanation is contamination of the Ly $\alpha$ absorption signal by diffuse Ly $\alpha$ emission associated with the extended Ly $\alpha$ halo of the foreground galaxy scattering into the slit apertures used to measure the spectra of the background galaxies. Estimates of the expected effect of Ly $\alpha$ emission contamination on measurements of $W_{\lambda}(\operatorname{Ly} \alpha)$ suggest that it should be non-negligible for any sample that uses background sources that are comparably bright to the foreground galaxies being probed. (Section 4.3)

(vi) Matching the $\tau_{\text {ap }}-D$ profile in $D_{\text {LOS }}$ and $D_{\text {tran }}$ axes shows strong redshift-space distortion at small $D$, and similar $\tau_{\text {ap profiles }}$ in the two directions at large $D$, suggesting that the redshift-space distortion becomes less prominent as $D \gtrsim 500 \mathrm{pkpc}$. (Section 4.5 )

(vii) The range of projected distance $50 \lesssim D_{\text {tran }} / \mathrm{pkpc} \lesssim 150$ marks a transition in both the $W_{\lambda}(\operatorname{Ly} \alpha)-D_{\text {tran }}$ relation, and the $2 \mathrm{D}$ $\tau_{\text {ap }}$ map, suggesting that outflows gradually cede to infall as the dominant source of absorbing gas within that range. This inference is also supported by the narrow velocity profile of absorption in the same range of $D_{\text {tran }}$, consistent with the effective resolution of the observed map, indicating a local minimum dispersion in $v_{\mathrm{LOS}}$ where the confluence of infall, outflow, and Hubble expansion create a caustic-like feature in $v_{\text {LOS }}$ space. (Section 4.2)

Our results for the spatial distribution and kinematics of $\mathrm{HI}$ could be compared with cosmological zoom-in simulations to test additional physical effects or feedback prescriptions (e.g. Hummels et al. 2013), and it is certainly possible to devise more realistic semi-analytical CGM models. Meanwhile, the clear distinction between outflow and inflow in the $v_{\text {LOS }}-D_{\text {tran }}$ space and the transition $D_{\text {tran }}$ between the two provides vital information on the interaction between galaxies and their surroundings during the periods of rapid galaxy growth.

Similar observations using metal-line absorption from CGM gas can provide information on gas with a wider range of physical conditions, further constraining the distribution, kinematics, and physical conditions of baryons around galaxies. It will also be intriguing to compare the $\mathrm{HI}$ kinematics in absorption and emission for the same galaxies with aid of deep IFU spectroscopy, which will provide more nuanced view of the structure and kinematics of the CGM and how it affects the radiative transfer of Ly $\alpha$. We are pursuing both of these approaches in forthcoming work.

\section{ACKNOWLEDGEMENTS}

Based on data obtained at the W. M. Keck Observatory, which is operated as a scientific partnership among the California Institute of Technology, the University of California, and the National Aeronautics and Space Administration. The Observatory was made possible by the generous financial support of the W. M. Keck Foundation.

This paper has included data obtained using Keck/LRIS (Oke et al. 1995; Steidel et al. 2004; Rockosi et al. 2010) and Keck/MOSFIRE (McLean et al. 2010, 2012). We thank the W. M. Keck Observatory staff for their assistance with the observations over two decades.

The following software packages have been crucial to preparing for this paper: the IDL Astronomy User's Library, ${ }^{17}$ the Coyote IDL library, ${ }^{18}$ ASTROPY (Astropy Collaboration 2018), EMCEE (ForemanMackey et al. 2013), THE YT PROJECT (Turk et al. 2011), and TRIDENT (Hummels, Smith \& Silvia 2017).

This work was supported in part by grant AST-1313472 from the US NSF, and by a grant from the Caltech/JPL President's

\footnotetext{
${ }^{17}$ https://idlastro.gsfc.nasa.gov/

${ }^{18}$ http://www.idlcoyote.com/
} 
and Director's Program. CAFG was supported by NSF through grants AST-1517491, AST-1715216, and CAREER award AST1652522, by NASA through grant 17-ATP17-0067, by STScI through grants HST-GO-14681.011, HST-GO-14268.022-A, and HST-AR14293.001-A, and by a Cottrell Scholar Award from the Research Corporation for Science Advancement.

We would like to thank the anonymous referee for providing valuable feedback. YC would like to thank Hongjie Zhu, for her continuous encouragements when preparing for this paper. We would like to acknowledge Yiqiu Ma, E. Sterl Phinney, and Mateusz Matuszewski, for their constructive discussions. Finally, we thank collaborators Kurt L. Adelberger, Matthew P. Hunt, David R. Law, Olivera Rakic, and Monica L. Turner for their contributions to the KBSS survey over the course of nearly two decades.

\section{DATA AVAILABILITY}

The processed data underlying this article are available on the KBSS website (http://ramekin.caltech.edu/KBSS) with DOI: 10.22002/D1.1458.

\section{REFERENCES}

Adelberger K. L., Steidel C. C., Shapley A. E., Pettini M., 2003, ApJ, 584, 45

Adelberger K. L., Steidel C. C., Shapley A. E., Hunt M. P., Erb D. K., Reddy N. A., Pettini M., 2004, ApJ, 607, 226

Adelberger K. L., Steidel C. C., Pettini M., Shapley A. E., Reddy N. A., Erb D. K., 2005a, ApJ, 619, 697

Adelberger K. L., Shapley A. E., Steidel C. C., Pettini M., Erb D. K., Reddy N. A., 2005b, ApJ, 629, 636

Anglés-Alcázar D., Faucher-Giguère C.-A., Kereš D., Hopkins P. F., Quataert E., Murray N., 2017, MNRAS, 470, 4698

Astropy Collaboration, 2018, ApJ, 156, 123

Bahcall J. N., Spitzer L. J., 1969, ApJ, 156, L63

Bielby R. M. et al., 2017, MNRAS, 471, 2174

Burchett J. N. et al., 2016, ApJ, 832, 124

Chen H.-W., Mulchaey J. S., 2009, ApJ, 701, 1219

Chen H., Lanzetta K. M., Webb J. K., Barcons X., 2001, ApJ, 559, 654

Chen H.-W., Prochaska J. X., Weiner B. J., Mulchaey J. S., Williger G. M., 2005, ApJ, 629, L25

Chen H.-W., Wild V., Tinker J. L., Gauthier J.-R., Helsby J. E., Shectman S. A., Thompson I. B., 2010, ApJ, 724, L176

Crighton N. H. M. et al., 2011, MNRAS, 414, 28

Dijkstra M., 2014, PASA, 31, e040

Erb D. K., Shapley A. E., Pettini M., Steidel C. C., Reddy N. A., Adelberger K. L., 2006a, ApJ, 644, 813

Erb D. K., Steidel C. C., Shapley A. E., Pettini M., Reddy N. A., Adelberger K. L., 2006b, ApJ, 646, 107

Erb D. K., Steidel C. C., Shapley A. E., Pettini M., Reddy N. A., Adelberger K. L., 2006c, ApJ, 647, 128

Erb D. K., Steidel C. C., Chen Y., 2018, ApJ, 862, L10

Faucher-Giguère C.-A., Prochaska J. X., Lidz A., Hernquist L., Zaldarriaga M., 2008, ApJ, 681, 831

Faucher-Giguère C.-A., Kereš D., Dijkstra M., Hernquist L., Zaldarriaga M., 2010, ApJ, 725, 633

Faucher-Giguère C.-A., Hopkins P. F., Kereš D., Muratov A. L., Quataert E., Murray N., 2015, MNRAS, 449, 987

Faucher-Giguère C.-A., Feldmann R., Quataert E., Kereš D., Hopkins P. F., Murray N., 2016, MNRAS, 461, L32

Foreman-Mackey D., Hogg D. W., Lang D., Goodman J., 2013, PASP, 125, 306

Franx M., Illingworth G. D., Kelson D. D., van Dokkum P. G., Tran K.-V., 1997, ApJ, 486, L75

Hafen Z. et al., 2019, MNRAS, 488, 1248
Hopkins P. F., Kereš D., Oñorbe J., Faucher-Giguère C.-A., Quataert E., Murray N., Bullock J. S., 2014, MNRAS, 445, 581

Hopkins P. F. et al., 2018, MNRAS, 480, 800

Hummels C. B., Bryan G. L., Smith B. D., Turk M. J., 2013, MNRAS, 430, 1548

Hummels C. B., Smith B. D., Silvia D. W., 2017, ApJ, 847, 59

Hummels C. B. et al., 2019, ApJ, 882, 156

Ji S. et al., 2020, MNRAS, 496, 4221

Johnson S. D., Chen H.-W., Mulchaey J. S., 2015, MNRAS, 449, 3263

Johnson S. D., Chen H.-W., Mulchaey J. S., Schaye J., Straka L. A., 2017, ApJ, 850, L10

Kaiser N., 1987, MNRAS, 227, 1

Kakiichi K., Dijkstra M., 2018, MNRAS, 480, 5140

Klypin A., Yepes G., Gottlöber S., Prada F., Heß S., 2016, MNRAS, 457, 4340

Kriek M. et al., 2015, ApJS, 218, 15

Lanzetta K. M., Bowen D. V., Tytler D., Webb J. K., 1995, ApJ, 442, 538

Law D. R., Steidel C. C., Shapley A. E., Nagy S. R., Reddy N. A., Erb D. K., 2012, ApJ, 745, 85

Lowenthal J. D. et al., 1997, ApJ, 481, 673

Madau P., Dickinson M., 2014, ARA\&A, 52, 415

McLean I. S. et al., 2010, in McLean I. S., Ramsay S. K., Takami H., eds Proc. SPIE Conf. Ser. Vol. 7735, Ground-based and Airborne Instrumentation for Astronomy III. SPIE, Bellingham, p. 77351E

McLean I. S. et al., 2012, in McLean I. S., Ramsay S. K., Takami H., eds, Proc. SPIE Conf. Ser. Vol. 8446, Ground-based and Airborne Instrumentation for Astronomy IV. SPIE, Bellingham, p. 84460J

Mitchell P. D., Schaye J., Bower R. G., Crain R. A., 2020, MNRAS, 494, 3971

Morris S. L., Jannuzi B. T., 2006, MNRAS, 367, 1261

Morrissey P. et al., 2018, ApJ, 864, 93

Muratov A. L., Kereš D., Faucher-Giguère C.-A., Hopkins P. F., Quataert E., Murray N., 2015, MNRAS, 454, 2691

Navarro J. F., Frenk C. S., White S. D. M., 1996, ApJ, 462, 563

Nelson D. et al., 2019, MNRAS, 490, 3234

Nielsen N. M., Kacprzak G. G., Pointon S. K., Churchill C. W., Murphy M. T., 2018, ApJ, 869, 153

Oke J. B., Korycansky D. G., 1982, ApJ, 255, 11

Oke J. B. et al., 1995, PASP, 107, 375

Oppenheimer B. D., Davé R., Kereš D., Fardal M., Katz N., Kollmeier J. A., Weinberg D. H., 2010, MNRAS, 406, 2325

Pettini M., Shapley A. E., Steidel C. C., Cuby J., Dickinson M., Moorwood A. F. M., Adelberger K. L., Giavalisco M., 2001, ApJ, 554, 981

Prochaska J. X., Weiner B., Chen H. W., Mulchaey J., Cooksey K., 2011, ApJ, 740, 91

Rakic O., Schaye J., Steidel C. C., Rudie G. C., 2012, ApJ, 751, 94

Rakic O., Schaye J., Steidel C. C., Booth C. M., Dalla Vecchia C., Rudie G. C., 2013, MNRAS, 433, 3103

Rauch M., Sargent W. L. W., Barlow T. A., 1999, ApJ, 515, 500

Reddy N. A., Steidel C. C., Fadda D., Yan L., Pettini M., Shapley A. E., Erb D. K., Adelberger K. L., 2006, ApJ, 644, 792

Reddy N. A., Steidel C. C., Pettini M., Adelberger K. L., Shapley A. E., Erb D. K., Dickinson M., 2008, ApJS, 175, 48

Reddy N. A., Pettini M., Steidel C. C., Shapley A. E., Erb D. K., Law D. R., 2012, ApJ, 754, 25

Rockosi C. et al., 2010, in McLean I. S., Ramsay S. K., Takami H., eds, Proc. SPIE Conf. Ser. Vol. 7735, Ground-based and Airborne Instrumentation for Astronomy III. SPIE, Belligham, p. 77350R

Rubin K. H. R., Diamond-Stanic A. M., Coil A. L., Crighton N. H. M., Stewart K. R., 2018, ApJ, 868, 142

Rudie G. C. et al., 2012a, ApJ, 750, 67

Rudie G. C., Steidel C. C., Pettini M., 2012b, ApJ, 757, L30

Rudie G. C., Steidel C. C., Shapley A. E., Pettini M., 2013, ApJ, 769, 146

Rudie G. C., Steidel C. C., Pettini M., Trainor R. F., Strom A. L., Hummels C. B., Reddy N. A., Shapley A. E., 2019, ApJ, 885, 61

Ryan-Weber E. V., 2006, MNRAS, 367, 1251

Shapley A. E., Steidel C. C., Pettini M., Adelberger K. L., 2003, ApJ, 588, 65 
Shapley A. E., Steidel C. C., Erb D. K., Reddy N. A., Adelberger K. L., Pettini M., Barmby P., Huang J., 2005, ApJ, 626, 698

Shone A. M., Morris S. L., Crighton N., Wilman R. J., 2010, MNRAS, 402, 2520

Steidel C. C., Giavalisco M., Pettini M., Dickinson M., Adelberger K. L., 1996, ApJ, 462, L17

Steidel C. C., Adelberger K. L., Shapley A. E., Pettini M., Dickinson M., Giavalisco M., 2003, ApJ, 592, 728

Steidel C. C., Shapley A. E., Pettini M., Adelberger K. L., Erb D. K., Reddy N. A., Hunt M. P., 2004, ApJ, 604, 534

Steidel C. C., Erb D. K., Shapley A. E., Pettini M., Reddy N. A., Bogosavljević M., Rudie G. C., Rakic O., 2010, ApJ, 717, 289

Steidel C. C., Bogosavljević M., Shapley A. E., Kollmeier J. A., Reddy N. A., Erb D. K., Pettini M., 2011, ApJ, 736, 160

Steidel C. C. et al., 2014, ApJ, 795, 165

Steidel C. C., Bogosavljević M., Shapley A. E., Reddy N. A., Rudie G. C., Pettini M., Trainor R. F., Strom A. L., 2018, ApJ, 869, 123

Strickland D. K., Heckman T. M., 2009, ApJ, 697, 2030

Strickland D. K., Heckman T. M., Colbert E. J. M., Hoopes C. G., Weaver K. A., 2004, ApJ, 606, 829

Strom A. L., Steidel C. C., Rudie G. C., Trainor R. F., Pettini M., Reddy N. A., 2017, ApJ, 836, 164

Tejos N. et al., 2014, MNRAS, 437, 2017

Theios R. L., Steidel C. C., Strom A. L., Rudie G. C., Trainor R. F., Reddy N. A., 2019, ApJ, 871, 128

Trainor R. F., Steidel C. C., 2012, ApJ, 752, 39

Tumlinson J. et al., 2011, ApJ, 733, 111

Tumlinson J., Peeples M. S., Werk J. K., 2017, ARA\&A, 55, 389

Tummuangpak P., Bielby R. M., Shanks T., Theuns T., Crighton N. H. M., Francke H., Infante L., 2014, MNRAS, 442, 2094

Turk M. J., Smith B. D., Oishi J. S., Skory S., Skillman S. W., Abel T., Norman M. L., 2011, ApJS, 192, 9

Turner M. L., Schaye J., Steidel C. C., Rudie G. C., Strom A. L., 2014 MNRAS, 445, 794

Turner M. L., Schaye J., Steidel C. C., Rudie G. C., Strom A. L., 2015, MNRAS, 450, 2067
Turner M. L., Schaye J., Crain R. A., Rudie G., Steidel C. C., Strom A., Theuns T., 2017, MNRAS, 471, 690

Wisotzki L. et al., 2016, A\&A, 587, A98

\section{APPENDIX A: STACKING METHOD}

To optimize the SNR of stacked spectra, we tested several different stacking methods: (1) sigma-clipped mean, (2) iterative sigmaclipped mean, (3) min-max clipped mean, and (4) median. For all cases, the error was estimated from bootstrap resampling of the sightlines within each bin using 2000 realizations. We select two $D_{\text {tran }}$ bins - one with $\left(D_{\text {tran }} / \mathrm{pkpc}\right) \leq 100$ and the other with $500 \leq$ $\left(D_{\text {tran }} / \mathrm{pkpc}\right) \leq 550$ from the KGPS-Full sample to demonstrate the effect of clipping on strong and weak Ly $\alpha$ absorption in Fig. A1. $W_{\lambda}(\operatorname{Ly} \alpha)$ was measured in the same way as in Section 3.1. The SNR of the continua is defined as the median flux density divided by the median error within two windows of rest wavelength, [1207, 1211] $\AA$ and [1220, 1224] $\AA$.

While keeping most of the data points in the mean stacks does not alter the value of $W_{\lambda}(\operatorname{Ly} \alpha)$ for either strong or weak lines, it significantly affects the SNR. On the other hand, achieving SNR similar to that obtained for median stacks requires more aggressive rejection $(\sim 2 \sigma$ or $\sim 8$ per cent) for the sigma clipping and minmax rejection, respectively. For iterative sigma clipping, we find that the optimal SNR is achieved at $\sim 2.5 \sigma$, where the SNR of $W_{\lambda}(\operatorname{Ly} \alpha)$ for weak lines and that of the continuum near strong lines are close to those of the median stack. Otherwise, the SNR remains $\sim 10$ per cent smaller than for spectra combined using a median stack; this suggests that the data values at each wavelength pixel are not normally distributed, and therefore outliers are not easily removed by sigma clipping. Given that the spectral continuum of the pre-stacked spectra are not normalized, part of this is contributed by the variations of the stellar continuum in the spectra of the background galaxies.
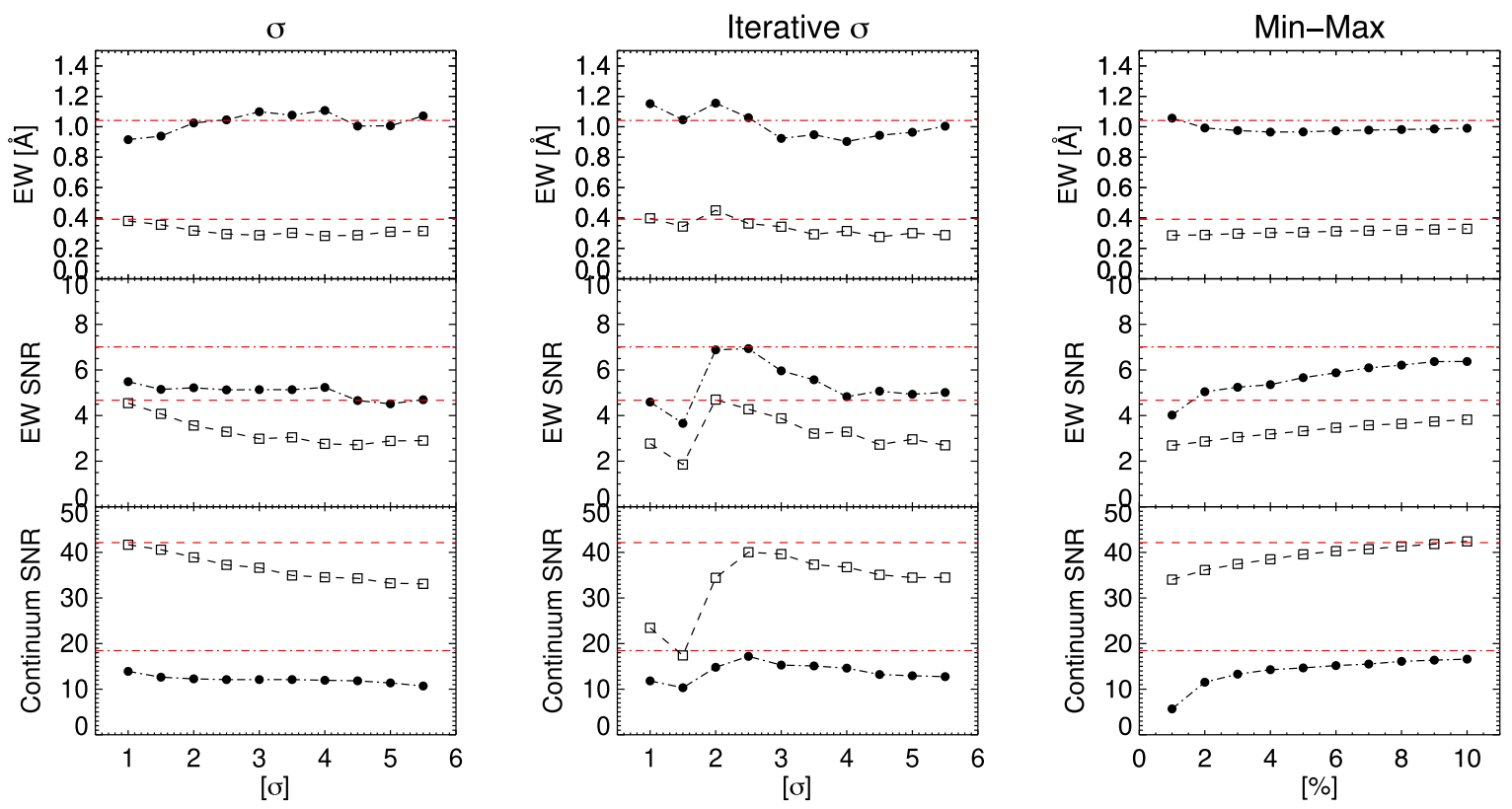

Figure A1. The impact of different stacking methods on $W_{\lambda}(\operatorname{Ly} \alpha)$ measurements: each panel shows $W_{\lambda}(\operatorname{Ly} \alpha)$ (top); the SNR of $W_{\lambda}($ Ly $\alpha)($ middle); the SNR of the continuum near Ly $\alpha$ (bottom). The three panels (left to right) show the results for sigma clipping, iterative sigma clipping, and min-max rejection. For min-max rejection, the $x$-axis shows the fraction of data points rejected from each side of the sample distribution. Within each panel, the black filled points correspond to a sample with $\left(D_{\text {tran }} / \mathrm{pkpc}\right) \leq 100$ and the skeletal boxes to a sample with $500 \leq\left(D_{\operatorname{tran}} / \mathrm{pkpc}\right) \leq 550$; the horizontal red lines show the corresponding values for a median stack with no other rejection algorithm applied. 
In addition, we find that in some regimes, the systematic effects of clipping methods have the opposite signs for strong and weak $W_{\lambda}($ Ly $\alpha)$ (top panel of Fig. A1). Since the median stack consistently returns values close to the optimal clipped mean, both in terms of measured $W_{\lambda}(\operatorname{Ly} \alpha)$ and SNR $\left(W_{\lambda}(\operatorname{Ly} \alpha)\right)$, we subsequently adopted the median stack for producing all composite spectra used in this paper.

\section{APPENDIX B: EFFECTIVE SPECTRAL RESOLUTION IN COMPOSITE SPECTRA}

Having a reliable measurement of the effective spectral resolution of the stacked spectra is crucial because some of the features in the 2D map are only marginally resolved. We stack spectra from the KGPS sample in the rest frame of $z_{\mathrm{bg}}$ and use the strong down-thebarrel UV features to estimate the effective resolution. We used the $\mathrm{C}$ II $\lambda 1334$ line since it does not suffer from significant contamination from other lines, and has the most consistent $W_{\lambda}$ (RMS $\lesssim 5$ per cent) in the stacks of different subsamples.

Assuming that the observed line width can be expressed as

$$
\begin{aligned}
\sigma_{\mathrm{obs}} & =\sqrt{\sigma_{0}^{2}+\sigma_{\mathrm{eff}}^{2}} \\
& =\sqrt{\sigma_{0}^{2}+\sigma_{\mathrm{inst}}^{2}+f_{\mathrm{zuv}} \sigma_{\mathrm{zuv}}^{2}},
\end{aligned}
$$

where $\sigma_{0}$ is the intrinsic width, $\sigma_{\text {eff }}$ is the effective velocity resolution, $\sigma_{\text {inst }}$ is the resolution of the spectrograph, and $f_{\text {zuv }}$ is the fraction of spectra in stacks that use calibration from $z_{\mathrm{Ly} \alpha}$ and $z_{\mathrm{abs}}$ as $z_{\mathrm{sys}}$, and $\sigma_{\text {zuv }}$ is the uncertainty of the calibration in velocity space. In this case, $\sigma_{\text {inst }}$ and $\sigma_{\text {zuv }}$ can be estimated separately. We assume that the redshift uncertainty is negligible when using $z_{\text {neb }}$ as $z_{\text {sys }}$.

Since essentially all our LRIS observations used either the $400 / 3400$ or $600 / 4000$ grisms, $\sigma_{\text {inst }}$ can be further divided into

$\sigma_{\text {inst }}=\sqrt{f_{400} \sigma_{400}^{2}+f_{600} \sigma_{600}^{2}}$,

where $\sigma_{400}$ and $\sigma_{600}$ are the instrument resolution for the $400 / 3400$ and 600/4000 grisms, and $f_{400}$ and $f_{600}$ are the fractions of spectra observed with the two grisms in the stack. To measure the absolute values of $\sigma_{400}$ and $\sigma_{600}$, we constructed 11 samples. Each has different ratio of $f_{400} / f_{600}$, ranging from 100 per cent of the spectra observed by the $400 / 3400$ grism to 100 percent observed by the $600 / 4000$ grism. All are based on exactly the same 95 objects, which have been observed with both 400/3400 and 600/4000 grisms, and have their systemic redshift measured from nebular lines. We fit the 10 stacks with non-zero contribution of $600 / 4000$ spectra by convolving the 100 per cent $600 / 4000$ stack with a Gaussian kernel, whose standard deviation $\left(\sigma_{\Delta}\right)$ is the only free parameter. The best- $\chi^{2}$ fits of $\sigma_{\Delta}$ are summarized in Fig. B1. Based on equation (B3)

$\sigma_{\Delta}=\sqrt{f_{400}\left(\sigma_{400}^{2}-\sigma_{600}^{2}\right)}$.

Therefore, $\sqrt{\sigma_{400}^{2}-\sigma_{600}^{2}}=146 \mathrm{~km} \mathrm{~s}^{-1}$, obtained by fitting the equation above to the measured points in Fig. B1.

Another ingredient for estimating the absolute value of $\sigma_{400}$ and $\sigma_{600}$ is the ratio of $\sigma_{400} / \sigma_{600}$. Fig. B2 shows the instrument resolution of the two grisms for an object with uniform illumination of a 1.2 arcsec slit, estimated from the arc spectra taken during afternoon calibrations. For a median redshift of 2.4 in our DTB stacks, the observed wavelength of C II $\lambda 1334$ is $4559 \AA$, which gives $\sigma_{400} / \sigma_{600}$ $\sim 1.69$. Therefore, $\sigma_{400}=181 \mathrm{~km} \mathrm{~s}^{-1}, \sigma_{600}=107 \mathrm{~km} \mathrm{~s}^{-1}$. This suggests a typical galaxy size of FWHM $=0.9$ arcsec. We then convert this measurement to the resolution that would be obtained

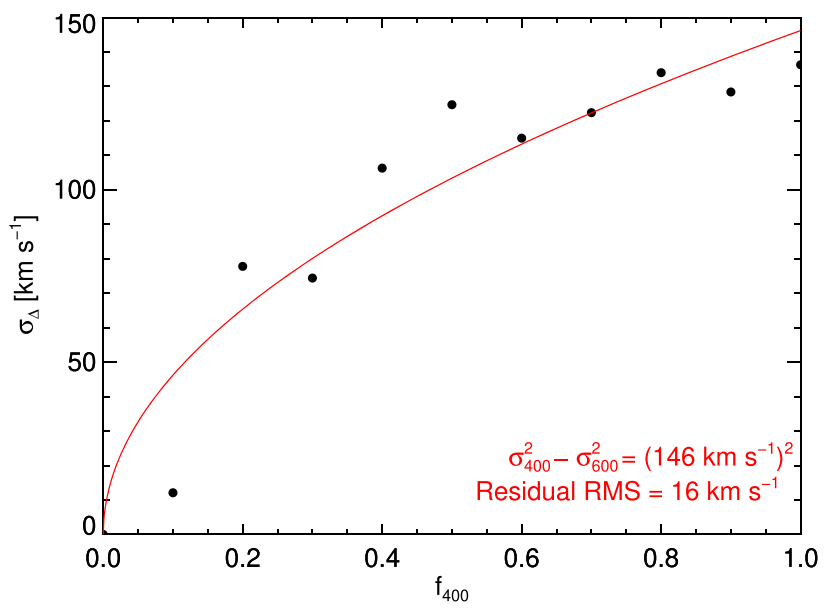

Figure B1. Width of the Gaussian kernel $\left(\sigma_{\Delta}\right)$ used in convolution to match the line profile of $\mathrm{C}$ II $\lambda 1334$, between stacks with pure $600 / 4000$ spectra and ones with a fraction of the $400 / 3400$ grism spectra $\left(f_{400}\right)$. The red line is the best-fitting model using equation (B4).

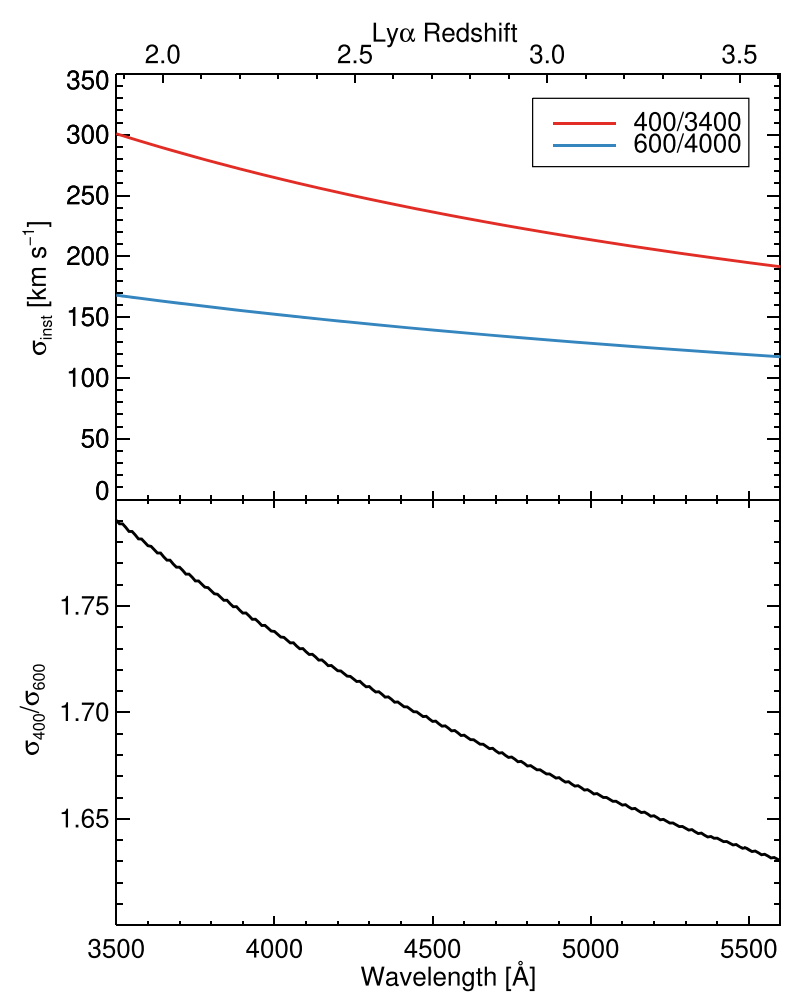

Figure B2. Spectroscopic resolution for 400/3400 and 600/4000 grisms plotted as $1 \sigma$ error in velocity space. Top: Absolute value estimated from arc spectra with 1.2-arcsec slit width. Bottom: Ratio between $\sigma_{400}$ and $\sigma_{600}$, which remains unchanged with varying object size in slits.

for Ly $\alpha$ at $z_{\text {med }}=2.2$. Assuming the $\lambda$-dependence of $\sigma$ as in Fig. B2, the velocity resolutions are $\sigma_{400}(\operatorname{Ly} \alpha)=211 \mathrm{~km} \mathrm{~s}^{-1}(R=604)$, and $\sigma_{600}(\operatorname{Ly} \alpha)=121 \mathrm{~km} \mathrm{~s}^{-1}(R=1055)$.

To test the reliability, we also made a stack with the same objects and combined all 400/3400 and 600/4000 spectra together, weighted by the number of objects instead of the number of spectra to repro- 
duce the scenario in Section 2.4. With 45 per cent of the weight given to $400 / 3400$ spectra, this yields a $\sigma_{\Delta}$ of $98 \mathrm{~km} \mathrm{~s}^{-1}$, consistent with equation (B4) within 10 per cent. For our typical foreground stacks in Section 2.4, 70 per cent of weight is contributed by $400 / 3400$ spectra, and 600/4000 spectra make up the remaining 30 percent. Therefore, the effective resolution, $\sigma_{\text {inst }}(\operatorname{Ly} \alpha)=189 \mathrm{~km} \mathrm{~s}^{-1}$.

The absolute value of $\sigma_{\text {zuv }}$ in equation (B1) can be obtained using a similar method. We constructed 11 stacks with 382 objects that

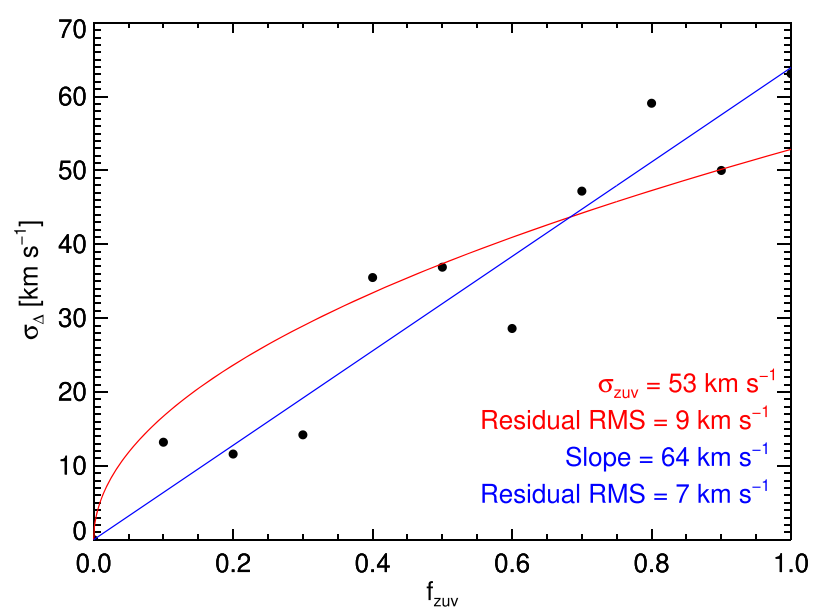

Figure B3. Similar to Fig. B1, but the $x$-axis is the fraction of objects in the stacks whose $z_{\text {sys }}$ is determined using rest-UV spectral features $\left(f_{\text {zuv }}\right)$. The red curve is the best fit of Fig. (B2), and the blue line is a simple linear fit. have redshift measurements with both nebular and rest-UV features. In these stacks, we randomly select $f_{\text {zuv }}$ fraction of objects and use the calibrated UV redshift $\left(z_{\mathrm{UV}}\right)$ instead of the precise $z_{\text {neb }}$. We use a similar method to fit the 10 stacks with non-zero $f_{\text {zuv }}$ by convolving a Gaussian kernel with the 100 percent $z_{\text {neb }}$ stack. The resulting kernel width as a function of $f_{\text {zuv }}$ is shown in Fig. B3. As can be seen in the figure, the relationship between $\sigma_{\Delta}$ and $f_{\text {zuv }}$ can be better represented by a linear function rather than a squareroot function, and the redshift errors associated with the use of UV features to estimate $z_{\text {sys }}$ are smaller than derived in Section 2.2. We suspect that the reason for the apparent discrepancy is that a large fraction of the derived $\sigma_{\mathrm{z}}$ in Section 2.2 is caused by noise in the measurement of individual UV features in the spectra of individual galaxies, whereas the calibration between $z_{\text {neb }}$ and $z_{\mathrm{UV}}$ effectively averages out such random noise. Nevertheless, we provide results for the two separate fits and use both in our final estimation. For a typical $f_{\text {zuv }}=50$ per cent in our foreground stacks for the KGPSFull sample, $\sigma_{\Delta}=37$ (square root) or 32 (linear) $\mathrm{km} \mathrm{s}^{-1}$. Putting all components together in equation (B1), the effective resolution for the KGPS-Full sample is $\sigma_{\text {eff }}(\operatorname{Ly} \alpha)=192 \mathrm{~km} \mathrm{~s}^{-1}$ for both the square root and the linear fit.

In summary, the effective spectral resolution for the KGPS- $z_{\text {neb }}$ and KGPS-Full sample is nearly identical: $\sigma_{\text {zneb }}=189 \mathrm{~km} \mathrm{~s}^{-1}$ $(R=676)$ and $\sigma_{\text {full }}=192 \mathrm{~km} \mathrm{~s}^{-1}(R=665)$, respectively. The uncertainty associated with the estimates is $\sim 15 \mathrm{~km} \mathrm{~s}^{-1}$, based on the rms residuals of the fits for $\sigma_{\Delta}-f_{400}$ and $\sigma_{\Delta}-f_{\text {zuv }}$ relations.

This paper has been typeset from a $\mathrm{T}_{\mathrm{E}} \mathrm{X} / \mathrm{LT} \mathrm{E} \mathrm{X}$ file prepared by the author. 